\title{
A stereoselective, multicomponent approach to quaternary substituted hydroindole scaffolds
}

Nicholas P. Massaro ${ }^{\mathrm{a}, \mathrm{b}}$ and Joshua G. Pierce*a,b

${ }^{a}$ Department of Chemistry, College of Sciences, NC State University, 2620 Yarbrough Drive, Raleigh, NC, USA ${ }^{\text {b }}$ Comparative Medicine Institute, NC State University, Raleigh, NC, USA

\section{Supporting Information}

\section{Table of Contents}

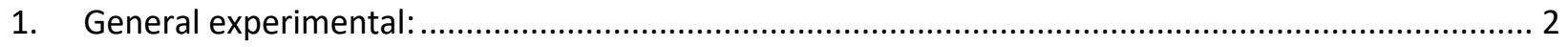

2. Synthesis of pyruvic acid methyl ester substrates 1a-1d........................................................ 3

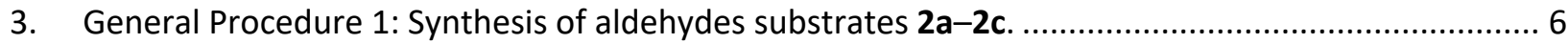

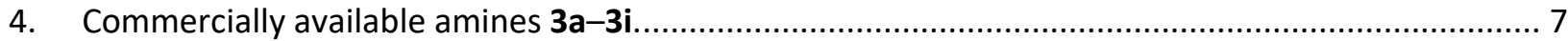

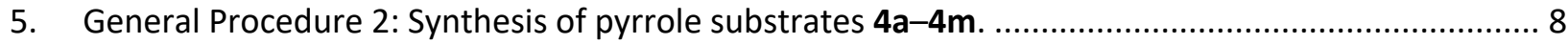

6. General Procedure 3: Synthesis of bicyclic pyrrole-2,3-diones 5a-5n. ....................................... 34 


\section{General experimental:}

General experimental procedures: All reactions were carried out under an inert argon atmosphere with anhydrous solvents under anhydrous conditions unless otherwise stated. All reactions, except those conducted in the presence of water were carried out in flame-dried apparatus. Yields refer to chromatographically yield, unless otherwise stated. Reactions were monitored by thin layer chromatography (TLC) analysis (pre-coated silica gel 60 F254 plates, $250 \mathrm{~mm}$ layer thickness) and visualization was accomplished with a $254 \mathrm{~nm}$ UV light and by staining with $\mathrm{KMnO}_{4}$, CAM, or PMA solutions with heating. Reactions were also monitored by LC-MS (2.6 mm C18 $50 \times 2.10 \mathrm{~mm}$ column). Flash chromatography on $\mathrm{SiO}_{2}$ was used to purify the crude reaction mixtures. Diastereomeric ratio was determined by crude ${ }^{1} \mathrm{H}$ NMR spectroscopy.

Materials: Tetrahydrofuran (THF) and dichloromethane $\left(\mathrm{CH}_{2} \mathrm{Cl}_{2}\right)$ were obtained by passing the previously degassed solvents through activated alumina columns under nitrogen atmosphere. Deuterated solvents (containing 0.03 to 0.05 vol \% tetramethylsilane, TMS) were purchased from Cambridge Isotope Laboratories. All other reagents were purchased from commercial chemical companies and used without further purification, unless otherwise stated.

Instrumentation: Infrared spectra were determined on the Agilent Cary 630 FTIR spectrometer and data are represented as frequency of absorption $\left(\mathrm{cm}^{-1}\right) .{ }^{1} \mathrm{H}$ and ${ }^{13} \mathrm{C} \mathrm{NMR} \mathrm{spectra} \mathrm{were} \mathrm{obtained} \mathrm{on} \mathrm{a} 500$ or 700 $\mathrm{MHz}$ instrument in $\mathrm{CDCl}_{3}$ unless otherwise noted. 1D nOe spectra were used to aid structure assignments when necessary. Chemical shifts $(\delta)$ were reported in parts per million (ppm) with the residual solvent peak used as an internal standard $\left(\mathrm{CDCl}_{3}{ }^{1} \mathrm{H} \mathrm{NMR}=7.26 \mathrm{ppm},{ }^{13} \mathrm{C} \mathrm{NMR}=77.00 \mathrm{ppm}\right)$ and multiplicities are reported as observed. The following abbreviations were used to explain NMR peak multiplicities: $s=$ singlet, $d=$ doublet, $t=$ triplet, $q=$ quartet, $p=$ pentet, $m=$ multiplet, $b r=$ broad. Low resolution mass spectra were obtained using electrospray ionization (ESI). High-resolution mass spectra were obtained on a high-resolution mass spectrometer - the Thermo Fisher Scientific Exactive Plus MS, a benchtop full-scan Orbitrap ${ }^{\mathrm{TM}}$ mass spectrometer - using Heated Electrospray lonization (HESI). The NC state small molecule $\mathrm{X}$-ray facility collected and analyzed all X-ray diffraction data. 
2. Synthesis of pyruvic acid methyl ester substrates $1 \mathrm{a}-1 \mathrm{~d}$.<smiles>COC(=O)/C(O)=C/C=C/c1ccccc1</smiles><smiles>COC(=O)/C(O)=C/c1ccccc1</smiles><smiles>COC(=O)/C(O)=C/c1ccc2c(c1)OCO2</smiles>

Compounds $\mathbf{1 a}$ and $\mathbf{1 b}$ were prepared using literature known protocol. Compound $\mathbf{1} \mathbf{d}$ is commercially available.

[1] Shymanska, N. V.; Pierce, J. G. Stereoselective Synthesis of Quaternary Pyrrolidine-2,3-diones and $\beta$-Amino Acids. Org. Lett. 2017, 19, 2961-2964.

[2] Commercially available as well as synthesized by Ullah, H.; Ferreira, A. V.; Bendassolli, J. A.; Rodrigues Jr., M. T.; Formiga, A. L. B.; Coelho, F. A Versatile Approach to Noncoded $\beta$-Hydroxy$\alpha$-amino Esters and $\alpha$-Amino Acids/Esters from Morita-Baylis-Hillman Adducts. Synthesis.

2015, 47, 113-123. 
Methyl (Z)-2-hydroxy-3-(2-nitrophenyl)acrylate (1c).<smiles>COC(=O)/C(O)=C/c1ccccc1[N+](=O)[O-]</smiles>

To a solution of 2-nitrophenylpyruvic acid in anhydrous DMF $(0.3 \mathrm{M})$ at $0{ }^{\circ} \mathrm{C}$ was added DBU $(357 \mu \mathrm{L}, 2.34$ mmol, 1.0 equiv.) followed by iodomethane ( $146 \mu \mathrm{L}, 2.34 \mathrm{mmol}, 1.0$ equiv.). The mixture was stirred at 0 ${ }^{\circ} \mathrm{C}$ for $3 \mathrm{~h}$. The crude reaction mixture was then poured over cold $\mathrm{HCl}$ solution (1 M) and extracted three times with $\mathrm{Et}_{2} \mathrm{O}$. The combined organic layers were washed with water, dried over $\mathrm{Na}_{2} \mathrm{SO}_{4}$, filtered and concentrated in volume. Crude was purified by flash column to afford $166.4 \mathrm{mg}$. $32 \%$ yield.

Yield: $32 \%, 166 \mathrm{mg}$.

Appearance: Pale yellow oil

$\boldsymbol{R}_{\mathrm{f}}=0.79$ (1:1 ethyl acetate:hexanes)

${ }^{1} \mathrm{H}$ NMR $\left(700 \mathrm{MHz}, \mathrm{CDCl}_{3}\right) \delta 8.16(\mathrm{~d}, J=8.3 \mathrm{~Hz}, 1 \mathrm{H}), 7.63(\mathrm{t}, J=7.7 \mathrm{~Hz}, 1 \mathrm{H}), 7.51(\mathrm{t}, J=7.8 \mathrm{~Hz}, 1 \mathrm{H}), 7.33(\mathrm{~d}$, $J=7.6 \mathrm{~Hz}, 1 \mathrm{H}), 4.53(\mathrm{~s}, 2 \mathrm{H}), 3.93(\mathrm{~s}, 3 \mathrm{H})$.

${ }^{13} \mathrm{C}$ NMR $\left(176 \mathrm{MHz}, \mathrm{CDCl}_{3}\right) \delta 189.0,160.9,148.2,133.9,133.7,129.0,128.9,125.5,53.3,44.5$.

IR (Diamond-ATR, neat) $\tilde{v}_{\text {max }}: 3457,2953,2855,1733,1522,1337,1256,1061,719$

HRMS (ESI) m/z: [M+H] $]^{+}$Calcd for $\mathrm{C}_{10} \mathrm{H}_{10} \mathrm{NO}_{5}$ 224.0554; Found 224.0556. 

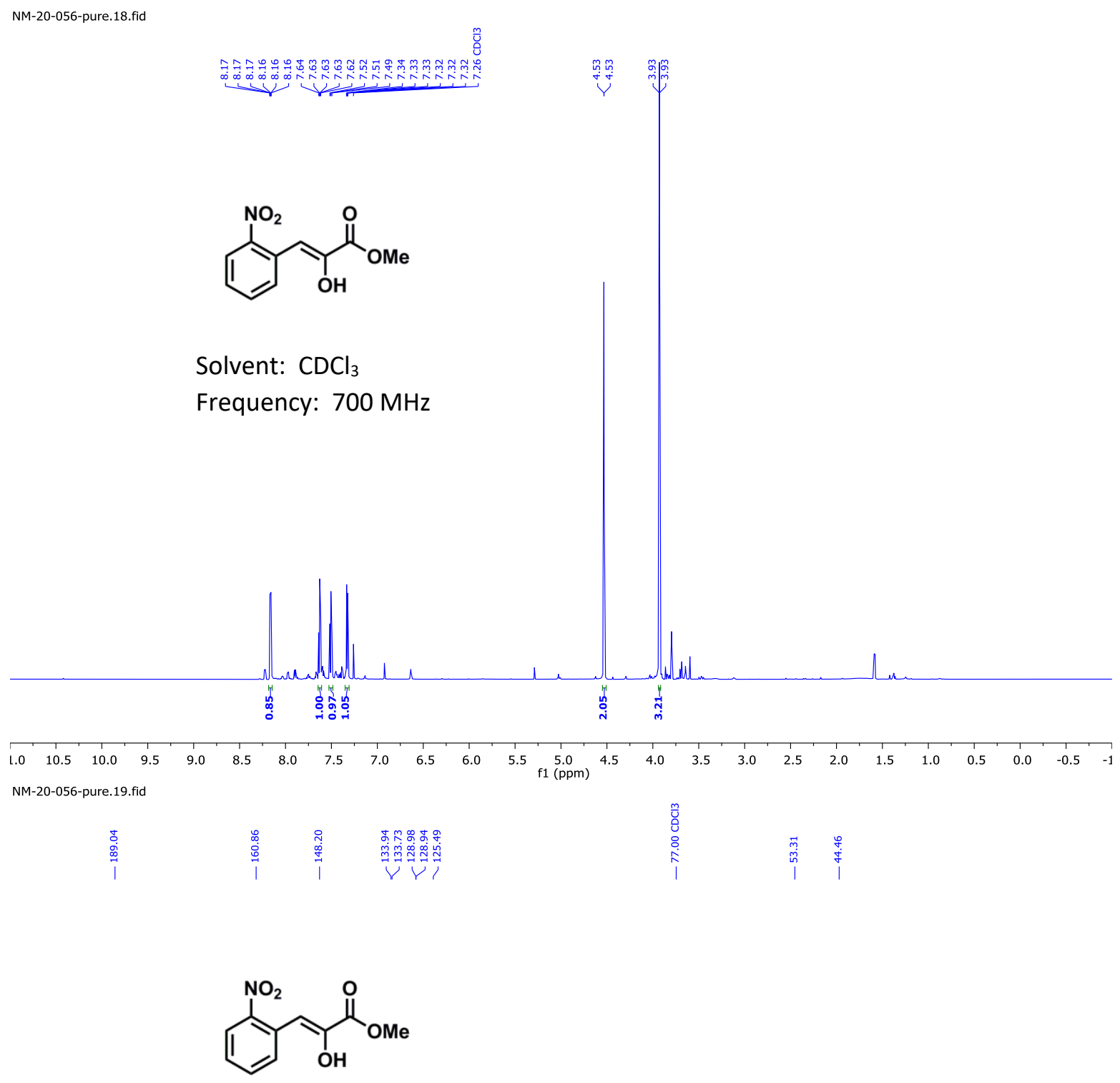

Solvent: $\mathrm{CDCl}_{3}$

Frequency: $176 \mathrm{MHz}$

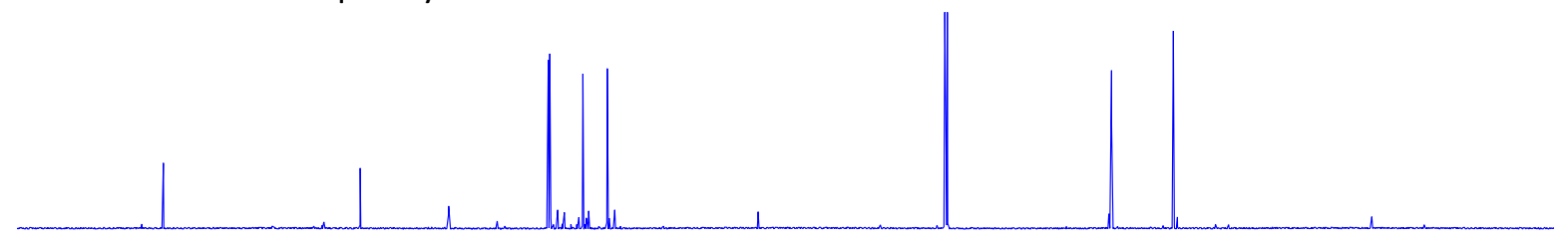

\begin{tabular}{lllllllllllllllllllllll}
\hline 10 & 200 & 190 & 180 & 170 & 160 & 150 & 140 & 130 & 120 & 110 & 100 & 90 & 80 & 70 & 60 & 50 & 40 & 30 & 20 & 10 & 0 & $-:$
\end{tabular}




\section{General Procedure 1: Synthesis of aldehydes substrates 2a-2c.}

To corresponding diol in a solution of dichloromethane at $0{ }^{\circ} \mathrm{C}$ was added triethylamine followed by tosyl chloride. Reaction was allowed to stir to room temperature until full consumption of tosyl chloride reagent. Reaction was quenched at $0{ }^{\circ} \mathrm{C}$ with the addition of saturated ammonium chloride solution. Crude mixture was extracted with dichloromethane three times. The combined organic layers were dried over sodium sulfate and concentrated to volume. The crude oil was purified by flash column chromatography using a gradient from hexanes to 1:1 hexanes:ethyl acetates obtaining an clear oil S2. To a round bottom flask containing dichloromethane under $\mathrm{N}_{2}$ was added oxalyl chloride. This solution was set to stirring at $-78{ }^{\circ} \mathrm{C}$, upon which anhydrous DMSO was added. This was allowed to stir for 15 minutes at this temperature. Then a solution of $\mathbf{S} 2$ in dichloromethane was added at $-78^{\circ} \mathrm{C}$. The reaction was then allowed to stir at this temperature for 1 hour. At this time, triethylamine was added, the dry-ice bath was removed and reaction was allowed to stir to room temperature for 1 hour. Reaction was then quenched with the addition of saturated ammonium chloride solution and extracted with dichloromethane three times. The combined organic layers were dried over sodium sulfate and concentrated to volume. Crude oil was then purified by flash column chromatography using a gradient of hexanes to 1:1 ethyl acetate hexanes yielding a clear oil in all cases.

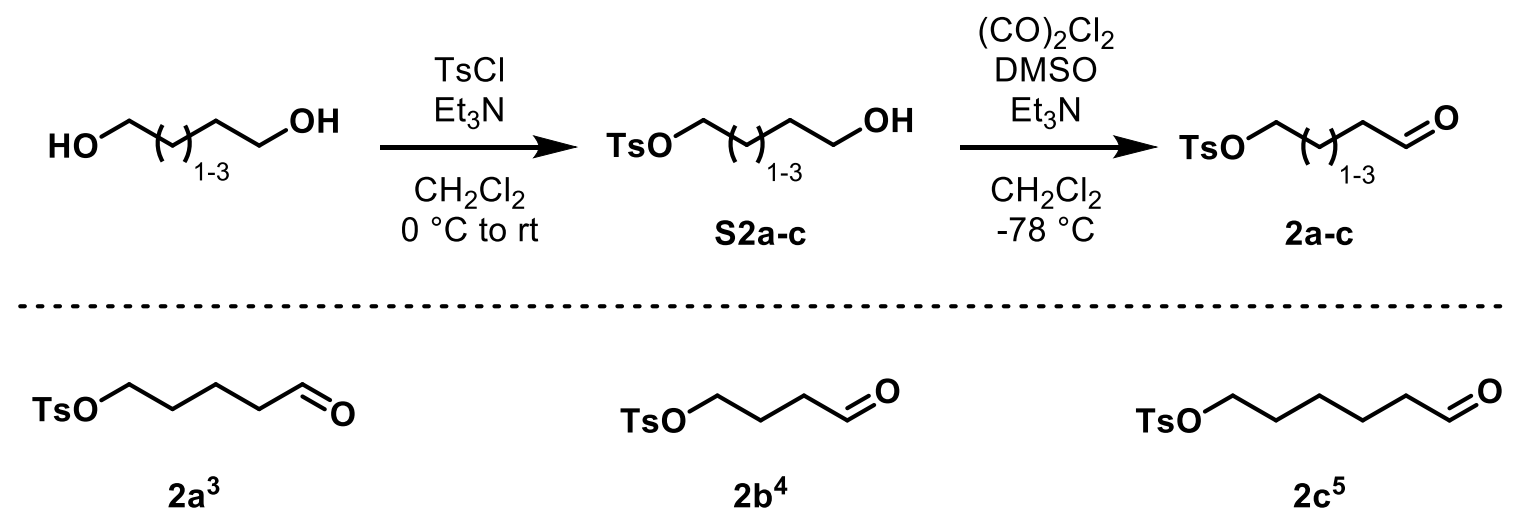

Compounds $\mathbf{2 a}, \mathbf{2} \mathbf{b}$ and $\mathbf{2 c}$ were prepared using general procedure 1 . Compounds $\mathbf{2 a}, \mathbf{2} \mathbf{b}$ and $\mathbf{2} \mathbf{c}$ matched literature known values. ${ }^{3-5}$

[3] Cooper, C. G. F.; Lee, E. R.; Silva, R. A.; Bourque, A. J.; Clark, S.; Katti, S.; Nivorozhkin, V. Process Development of a Potent Glucosylceramide Synthase Inhibitor. Org. Process Res. Dev. 2012, 16, 1090-1097.

[4] Ning, X.-S.; Wang, M.-M.; Yao, C.-Z.; Chen, X.-M.; Kang, Y.-B. tert-Butyl Nitrite: Organic Redox Cocatalyst for Aerobic Aldehyde-Selective Wacker-Tsuji Oxidation. Org. Lett. 2016, 18, 27002703.

[5] Disadee, W.; Ruchirawat, S. One-pot cascade synthesis of azabicycles via the nitro-Mannich reaction and N-alkylation. Org. Biomol. Chem. 2018, 16, 707-711. 
4. Commercially available amines 3a-3i.

$\mathrm{H}_{2} \mathrm{~N}-\mathrm{Dmb}$

$3 a^{6}$<smiles>NCc1ccc(Br)cc1</smiles>

$3 d^{9}$

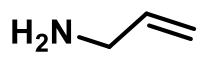

$3 g^{12}$<smiles>NCc1ccccc1[N+](=O)[O-]</smiles>

$3 b^{7}$<smiles>NCc1ccc2c(c1)OCO2</smiles>

$3 e^{10}$

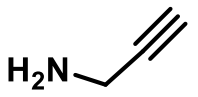

$3 h^{13}$

\section{$\mathrm{H}_{2} \mathrm{~N}-\mathrm{Bn}$}

$3 c^{8}$<smiles>NCc1cccs1</smiles>

$3 f^{11}$

$\mathrm{H}_{3} \mathrm{~N}-\mathrm{Me}$

$3 i^{14}$

Compounds $\mathbf{3 a}, \mathbf{3} \mathbf{c}-\mathbf{3 i}$ were purchased commercially. ${ }^{6,8-14}$ Compound $\mathbf{3 b}$ was prepared from 2nitrobenzylamine hydrochloride using a known literature protocol. ${ }^{7}$

[6] 2,4-dimethoxybenzylamine, 98\%, Alfa Aesar, Lot \#10163490

[7] Yawer, M. A.; Sleziakova, K.; Pavlovec, L.; Sindelar, V. Bambusurils Bearing Nitro Groups and Their Further Modifications. Eur. J. Org. Chem. 2018, 41-47.

[8] Benzylamine, 99\%, Sigma Aldrich, Lot \#SHBJ9249

[9] 4-bromobenzylamine, 97\%, Acros Organics, Lot \#A0366663

[10] Piperonylamine, 97\%, Sigma Aldrich, Lot \#STBJ1763

[11] 2-thiophenemethylamine, 97\%, Alfa Aesar, Lot \#10164904

[12] Allylamine, 99\%, Sigma Aldrich, Lot \#SHBG4366V

[13] Propargylamine, 99\%, Acros Organics, Lot \#A0379564

[14] Methylammonium chloride, 99\%, EMD Millipore, Lot \#S6896120813 


\section{General Procedure 2: Synthesis of pyrrole substrates $4 a-4 m$.}

A solution of the corresponding pyruvic methyl ester 1 (1.0 equiv) and aldehyde 2 (1.1 equiv) in anhydrous dichloromethane $(0.5 \mathrm{M})$ was added corresponding primary amine 3 (1.1 equiv) at $0{ }^{\circ} \mathrm{C} .{ }^{15}$ Reaction was allowed to stir to room temperature. Reaction was monitored by TLC for full consumption of pyruvic methyl ester 1. Reaction times ranged from 4 to 12 hours. Upon completion, reaction was concentrated to volume and immediately purified by flash column chromatography using a gradient from hexanes to 1:1 ethyl acetate:hexanes mixture yielding pyrrole substrate 4.

(E)-4-(1-(2,4-diMethoxybenzyl)-4-hydroxy-5-oxo-3-styryl-2,5-dihydro-1H-pyrrol-2-yl)butyl

4methylbenzenesulfonate (4a).<smiles>COc1ccc(CN2C(=O)C(O)=C(/C=C/c3ccccc3)C2CCCC[S+](F)(F)F)c(OC)c1</smiles>

Yield: $97.4 \mathrm{mg}, 71 \%$.

Appearance: off-white solid.

$\boldsymbol{R}_{\mathbf{f}}=0.50$ (1:1 ethyl acetate:hexanes).

${ }^{1} \mathrm{H}$ NMR $\left(700 \mathrm{MHz}, \mathrm{CDCl}_{3}\right) \delta 7.72(\mathrm{~d}, J=8.3 \mathrm{~Hz}, 2 \mathrm{H}), 7.43(\mathrm{~d}, J=7.7 \mathrm{~Hz}, 2 \mathrm{H}), 7.33(\mathrm{t}, J=7.7 \mathrm{~Hz}, 2 \mathrm{H}), 7.29$ (d, $J=8.1 \mathrm{~Hz}, 2 \mathrm{H}), 7.26-7.23(\mathrm{~m}, 1 \mathrm{H}), 7.15(\mathrm{~d}, J=8.0 \mathrm{~Hz}, 1 \mathrm{H}), 6.88(\mathrm{~d}, J=16.5 \mathrm{~Hz}, 1 \mathrm{H}), 6.73(\mathrm{~d}, J=16.5 \mathrm{~Hz}, 1 \mathrm{H})$, 6.46-6.43 (m, 2H), $4.91(\mathrm{~d}, J=15.0 \mathrm{~Hz}, 1 \mathrm{H}), 4.22(\mathrm{~d}, J=15.0 \mathrm{~Hz}, 1 \mathrm{H}), 4.12(\mathrm{t}, J=3.6 \mathrm{~Hz}, 1 \mathrm{H}), 3.92-3.88(\mathrm{~m}$, $2 \mathrm{H}), 3.83(\mathrm{~s}, 3 \mathrm{H}), 3.80(\mathrm{~s}, 3 \mathrm{H}), 2.41(\mathrm{~s}, 3 \mathrm{H}), 2.03-1.97(\mathrm{~m}, 1 \mathrm{H}), 1.83-1.77(\mathrm{~m}, 1 \mathrm{H}), 1.70-1.48(\mathrm{~m}, 3 \mathrm{H}), 0.96-$ $0.91(\mathrm{~m}, 2 \mathrm{H})$.

${ }^{13} \mathrm{C} \mathrm{NMR}\left(176 \mathrm{MHz}, \mathrm{CDCl}_{3}\right) \delta 166.8,160.7,158.3,144.7,142.7,137.0,132.9,131.1,131.0,129.8(2 \mathrm{C}), 128.7$ (2C), 128.0, $127.8(2 \mathrm{C}), 126.4(2 \mathrm{C}), 120.4,117.3,117.2,104.4,98.5,70.2,56.6,55.5,55.4,38.3,28.7,28.2$, 21.6, 17.4 .

IR (Diamond-ATR, neat) $\tilde{v}_{\text {max }}: 3039,2935,1652,1449,1353,1262,1175,933,817,660$.

HRMS (ESI) m/z: [M+H] $]^{+}$Calcd for $\mathrm{C}_{32} \mathrm{H}_{36} \mathrm{NO}_{7} \mathrm{~S}$ 578.2207; Found 578.2218. 


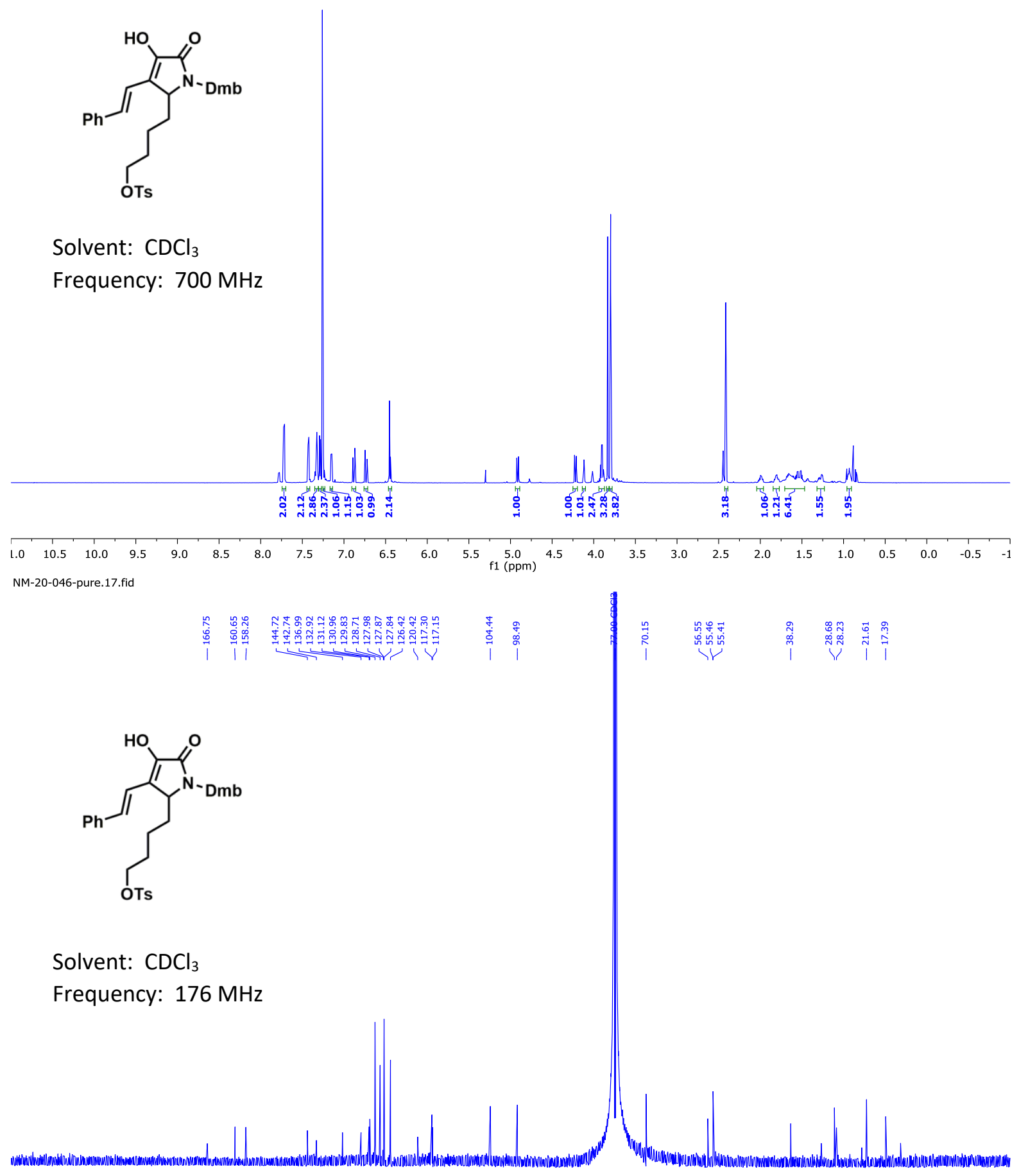

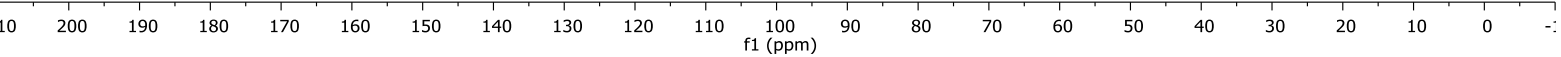


<smiles>O=C1C(O)=C(/C=C/c2ccccc2)C(CCCC[SnH3])N1Cc1ccccc1[N+](=O)[O-]</smiles>

Yield: $75.0 \mathrm{mg}, 56 \%$.

Appearance: orange oil.

$\boldsymbol{R}_{\mathrm{f}}=0.40$ (1:1 ethyl acetate:hexanes).

${ }^{1} \mathrm{H}$ NMR $\left(500 \mathrm{MHz}, \mathrm{CDCl}_{3}\right) \delta 8.57(\mathrm{br}, 1 \mathrm{H}), 7.96(\mathrm{~d}, J=7.9 \mathrm{~Hz}, 1 \mathrm{H}) 7.73(\mathrm{~d}, J=8.3 \mathrm{~Hz}, 2 \mathrm{H}), 7.61(\mathrm{td}, J=7.6$, $1.3 \mathrm{~Hz}, 1 \mathrm{H}), 7.45(\mathrm{dd}, J=8.2,6.7 \mathrm{~Hz}, 2 \mathrm{H}), 7.37(\mathrm{dd}, J=8.0,1.3 \mathrm{~Hz}, 1 \mathrm{H}), 7.33(\mathrm{t}, J=7.5 \mathrm{~Hz}, 2 \mathrm{H}), 7.31-7.22$ $(\mathrm{m}, 4 \mathrm{H}), 6.96(\mathrm{~d}, J=16.5 \mathrm{~Hz}, 1 \mathrm{H}), 6.80(\mathrm{~d}, J=16.6 \mathrm{~Hz}, 1 \mathrm{H}), 5.27(\mathrm{~d}, J=17.2 \mathrm{~Hz}, 1 \mathrm{H}), 4.72(\mathrm{~d}, J=16.9 \mathrm{~Hz}, 1 \mathrm{H})$, $4.29(\mathrm{t}, J=3.6 \mathrm{~Hz}, 1 \mathrm{H}), 3.89(\mathrm{td}, J=6.5,3.3 \mathrm{~Hz}, 2 \mathrm{H}), 2.41(\mathrm{~s}, 3 \mathrm{H}), 1.88(\mathrm{td}, J=8.5,3.6 \mathrm{~Hz}, 2 \mathrm{H}), 1.56-1.43(\mathrm{~m}$, $2 \mathrm{H}), 1.05-0.90(\mathrm{~m}, 2 \mathrm{H})$.

${ }^{13} \mathrm{C}$ NMR $\left(176 \mathrm{MHz}, \mathrm{CDCl}_{3}\right) \delta 168.1,148.2,144.7,142.9,136.7,133.9,132.8,132.2,131.8,129.8(2 \mathrm{C})$, $129.8,128.7$ (2C), 128.6, 128.2, 127.8 (2C), 126.5 (2C), 125.1, 122.4, 117.1, 69.9, 57.3, 40.8, 28.6, 28.5, 21.6, 17.5.

IR (Diamond-ATR, neat) $\tilde{v}_{\text {max }}:$ 3019, 2937, 2866, 1707, 1524, 1349, 1170, 939, 749, 660.

HRMS (ESI) m/z: [M+H] $]^{+}$Calcd for $\mathrm{C}_{30} \mathrm{H}_{31} \mathrm{~N}_{2} \mathrm{O}_{7} \mathrm{~S}$ 563.1847; Found 563.1861. 
<smiles>O=C1C(O)=C(C=Cc2ccccc2)C(CCCCO)N1Cc1ccccc1[N+](=O)[O-]</smiles>

Solvent: $\mathrm{CDCl}_{3}$

Frequency: $500 \mathrm{MHz}$

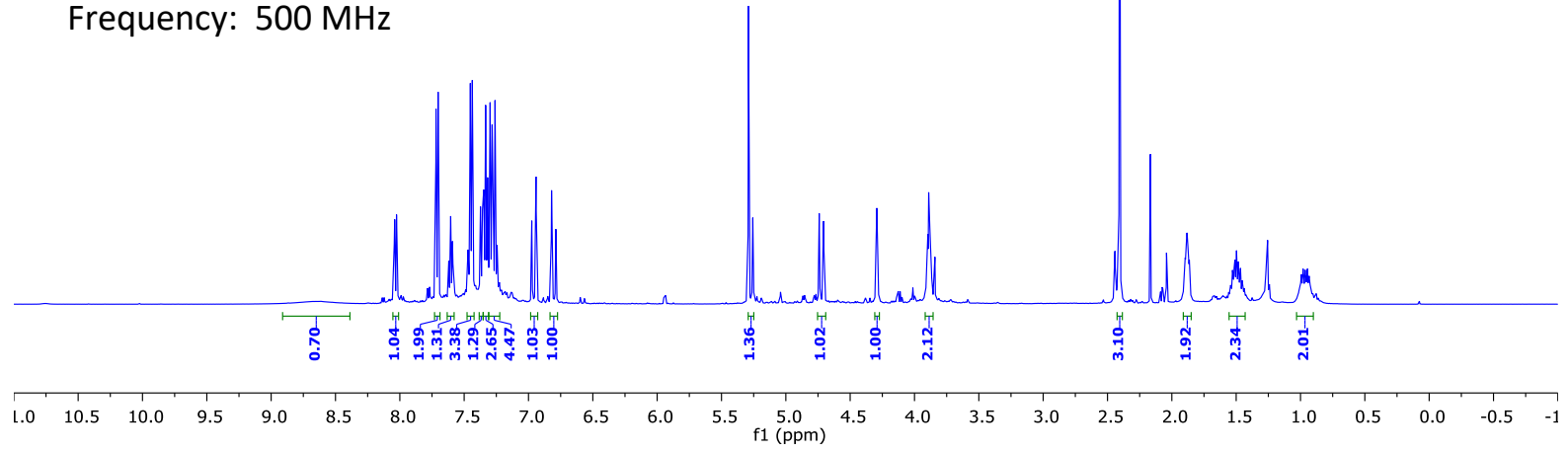

NM-20-028-C13.11.fid

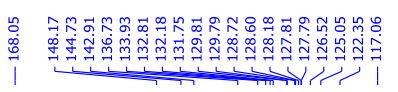<smiles>O=C1C(O)=C(C=Cc2ccccc2)C(CCCCO)N1Cc1ccccc1[N+](=O)[O-]</smiles>

Solvent: $\mathrm{CDCl}_{3}$

Frequency: $176 \mathrm{MHz}$

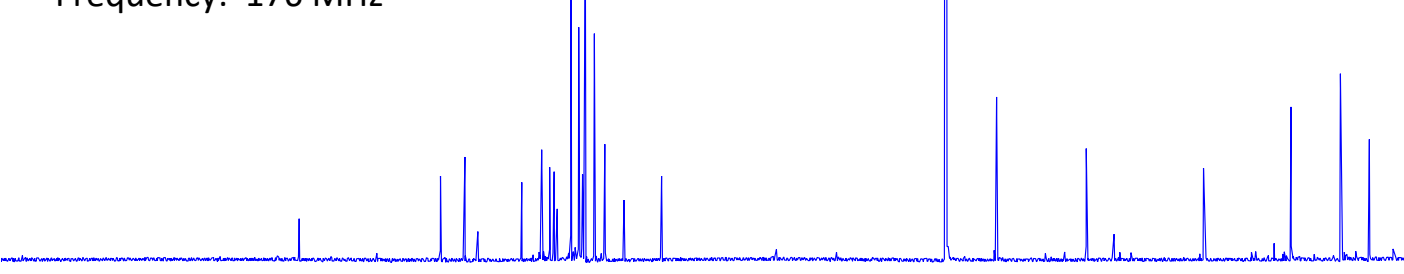

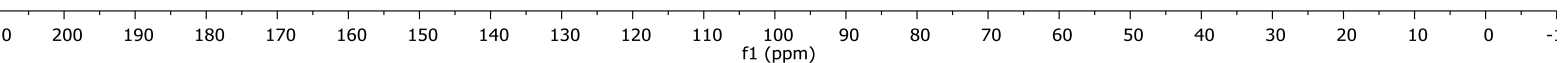


(E)-4-(1-Benzyl-4-hydroxy-5-oxo-3-styryl-2,5-dihydro-1H-pyrrol-2-yl)butyl 4-methylbenzenesulfonate (4c).<smiles>O=C1C(O)=C(/C=C/c2ccccc2)C(CCCCOC(F)(F)F)N1Cc1ccccc1</smiles>

Yield: $85.1 \mathrm{mg}, 69 \%$.

Appearance: orange waxy solid.

$\boldsymbol{R}_{\mathrm{f}}=0.26$ (1:1 ethyl acetate:hexanes).

${ }^{1} \mathbf{H}$ NMR $\left(500 \mathrm{MHz}, \mathrm{CDCl}_{3}\right) \delta 8.59(\mathrm{br}, 1 \mathrm{H}), 7.72(\mathrm{~d}, J=8.3 \mathrm{~Hz}, 2 \mathrm{H}), 7.42(\mathrm{~d}, J=7.5 \mathrm{~Hz}, 2 \mathrm{H}), 7.37-7.21(\mathrm{~m}$, $10 \mathrm{H}), 6.95(\mathrm{~d}, J=16.6 \mathrm{~Hz}, 1 \mathrm{H}), 6.74(\mathrm{~d}, J=16.6 \mathrm{~Hz}, 1 \mathrm{H}), 5.12(\mathrm{~d}, J=15.3 \mathrm{~Hz}, 1 \mathrm{H}), 4.20-4.14(\mathrm{~m}, 2 \mathrm{H}), 3.93-$ $3.82(\mathrm{~m}, 2 \mathrm{H}), 2.41(\mathrm{~s}, 3 \mathrm{H}), 1.86(\mathrm{dddd}, J=23.6,14.7,11.4,6.7 \mathrm{~Hz}, 2 \mathrm{H}), 1.57-1.38(\mathrm{~m}, 2 \mathrm{H}), 1.03-0.84(\mathrm{~m}$, $2 \mathrm{H})$.

${ }^{13} \mathrm{C}$ NMR $\left(176 \mathrm{MHz}, \mathrm{CDCl}_{3}\right) \delta 167.3,144.8,143.3,136.9,136.6,132.8,131.0,129.8(2 \mathrm{C}), 128.8(2 \mathrm{C}), 128.7$ (2C), $128.1(2 \mathrm{C}), 128.0,127.8(2 \mathrm{C}), 127.8,126.4(2 \mathrm{C}), 121.5,117.3,70.0,56.4,44.3,28.4,28.3,21.6,17.3$.

IR (Diamond-ATR, neat) $\tilde{v}_{\text {max }}: 3056,3030,2926,2866,1655,1450,1353,1174,939,731,697,660$.

HRMS (ESI) m/z: [M+H] $]^{+}$Calcd for $\mathrm{C}_{30} \mathrm{H}_{32} \mathrm{NO}_{5} \mathrm{~S}$ 518.1996; Found 518.2009. 
Feb13-2020-NM-20-027pure.12.fid
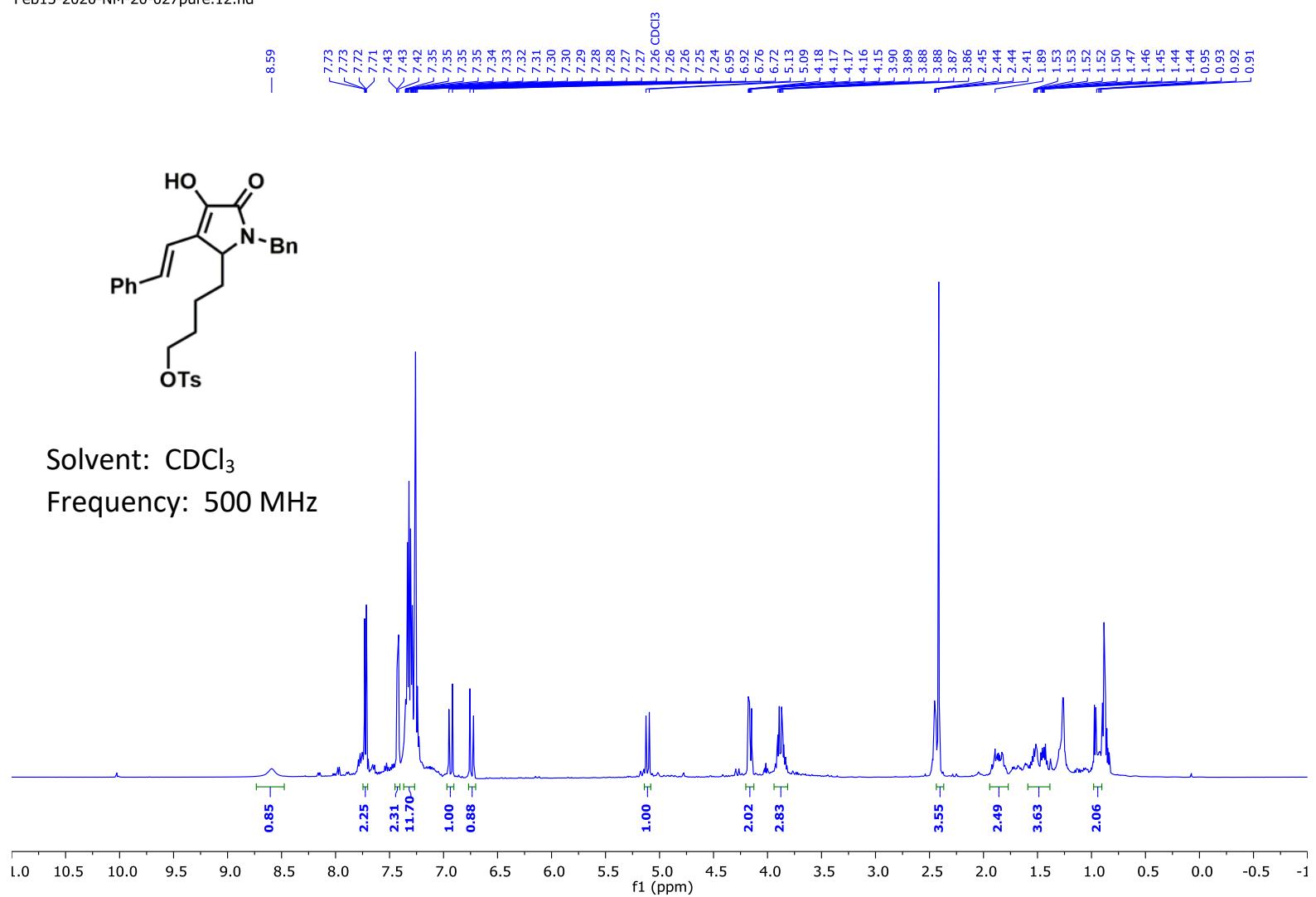

NM-20-027-C13.10.fid

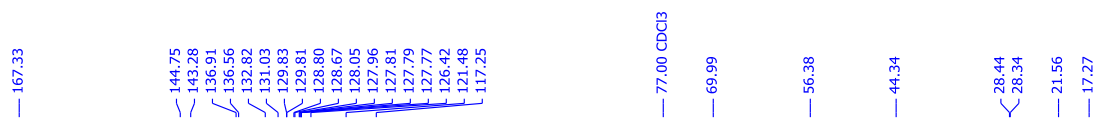<smiles>O=C1C(O)=CC(=CCCCO)C1CCCCCO</smiles>

Solvent: $\mathrm{CDCl}_{3}$

Frequency: $176 \mathrm{MHz}$

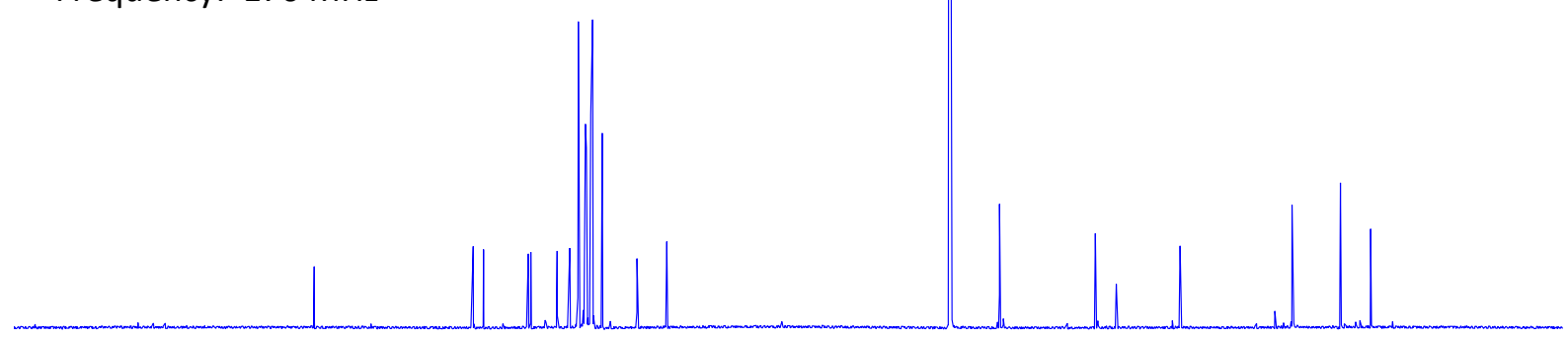

$\begin{array}{lllllllllllllllllllllll}10 & 200 & 190 & 180 & 170 & 160 & 150 & 140 & 130 & 120 & 110 & \underset{\mathrm{f} 1(\mathrm{ppm})}{100} & 90 & 80 & 70 & 60 & 50 & 40 & 30 & 20 & 10 & 0 & -.\end{array}$ 


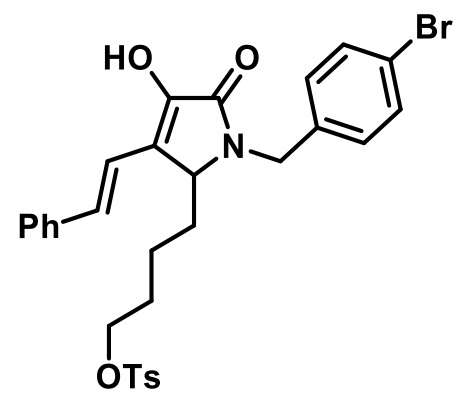

Yield: $83.6 \mathrm{mg}, 59 \%$.

Appearance: pale yellow greasy solid.

$\boldsymbol{R}_{\mathrm{f}}=0.29$ (1:1 ethyl acetate:hexanes).

${ }^{1} \mathrm{H}$ NMR $\left(500 \mathrm{MHz}, \mathrm{CDCl}_{3}\right) \delta 8.66(\mathrm{br} \mathrm{s}, 1 \mathrm{H}), 7.73(\mathrm{~d}, J=8.3 \mathrm{~Hz}, 2 \mathrm{H}), 7.46(\mathrm{~d}, J=8.5 \mathrm{~Hz}, 2 \mathrm{H}), 7.43(\mathrm{~d}, J=7.5$ $\mathrm{Hz}, 2 \mathrm{H}), 7.35-7.28(\mathrm{~m}, 5 \mathrm{H}), 7.15(\mathrm{~d}, J=8.2 \mathrm{~Hz}, 2 \mathrm{H}), 6.94(\mathrm{~d}, J=16.6 \mathrm{~Hz}, 1 \mathrm{H}), 6.75(\mathrm{~d}, J=16.6 \mathrm{~Hz}, 1 \mathrm{H}), 5.10$ $(\mathrm{d}, J=15.3 \mathrm{~Hz}, 1 \mathrm{H}), 4.14(\mathrm{t}, J=3.4 \mathrm{~Hz}, 1 \mathrm{H}), 4.09(\mathrm{~d}, J=15.4 \mathrm{~Hz}, 1 \mathrm{H}), 3.97-3.84(\mathrm{~m}, 2 \mathrm{H}), 2.42(\mathrm{~s}, 3 \mathrm{H}), 1.86$ (dddd, $J=25.8,14.3,8.0,3.2 \mathrm{~Hz}, 2 \mathrm{H}), 1.62-1.42(\mathrm{~m}, 2 \mathrm{H}), 1.05-0.89(\mathrm{~m}, 2 \mathrm{H})$.

${ }^{13} \mathrm{C}$ NMR $\left(176 \mathrm{MHz}, \mathrm{CDCl}_{3}\right) \delta 167.3,144.8,143.0,136.8,135.6,132.8,132.0$ (2C), 131.4, 129.9 (2C), 129.8 (2C), 128.7 (2C), 128.1, 127.8 (2C), 126.5 (2C), 121.8, 121.6, 117.1, 69.9, 56.3, 43.7, 28.4, 28.3, 21.6, 17.4 .

IR (Diamond-ATR, neat) $\tilde{v}_{\text {max }}:$ 3027, 2933, 2870, 1655, 1446, 1394, 1353, 1174, 962, 939, 753, 663.

HRMS (ESI) m/z: [M+H] ${ }^{+}$Calcd for $\mathrm{C}_{30} \mathrm{H}_{31} \mathrm{BrNO}_{5} \mathrm{~S}$ 596.1101; Found 596.1118. 


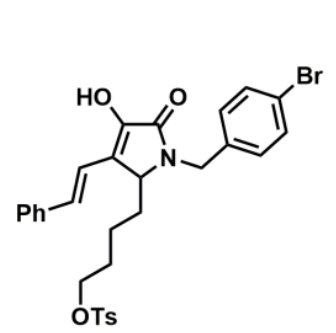

Solvent: $\mathrm{CDCl}_{3}$

Frequency: $500 \mathrm{MHz}$

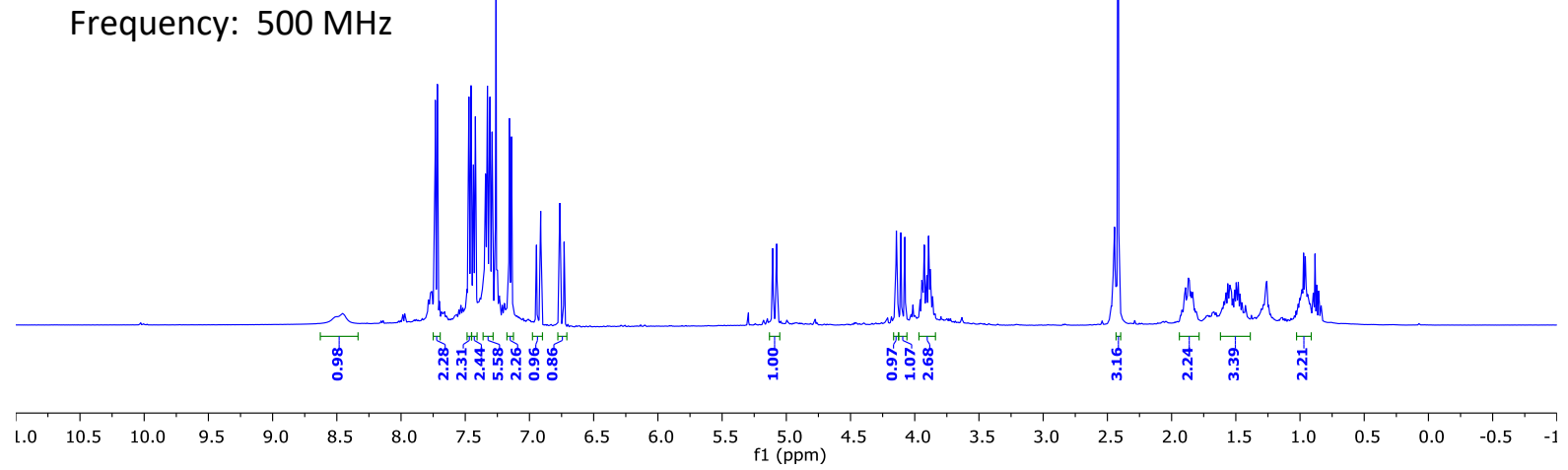

NM-20-030-C13.12.fid
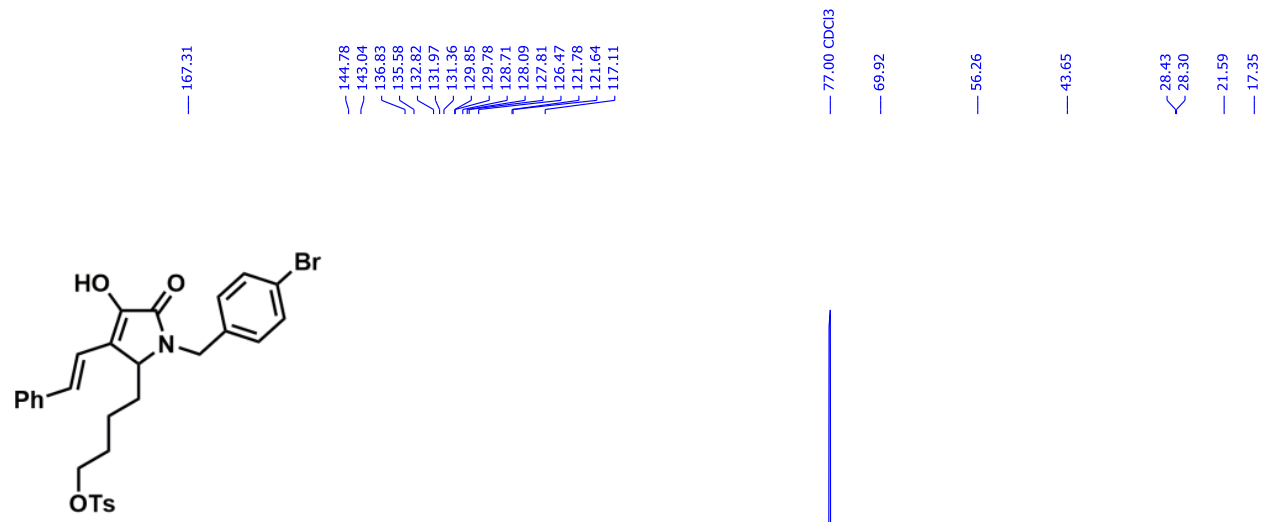

Solvent: $\mathrm{CDCl}_{3}$

Frequency: $176 \mathrm{MHz}$

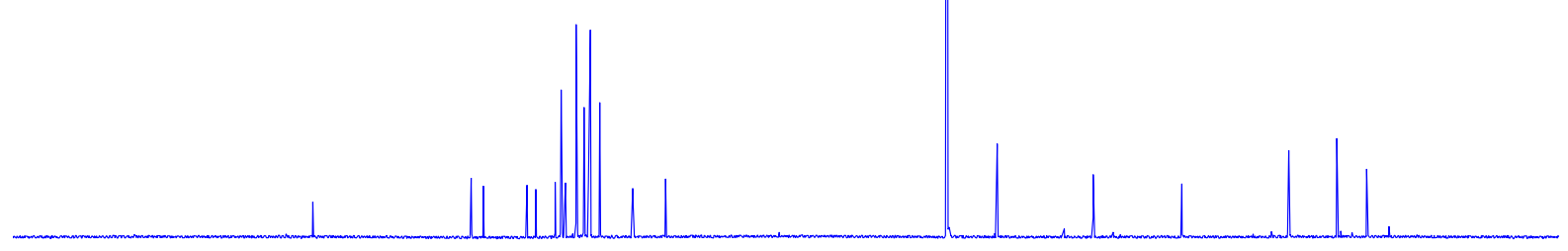

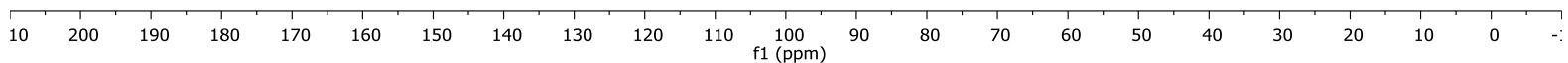


(E)-4-(1-(Benzo[d][1,3]dioxol-5-ylmethyl)-4-hydroxy-5-oxo-3-styryl-2,5-dihydro-1H-pyrrol-2-yl)butyl 4methylbenzenesulfonate (4e).<smiles>O=C1C(O)=C(/C=C/c2ccccc2)C(CCCC[SeH])N1Cc1ccc2c(c1)OCO2</smiles>

Yield: $86.9 \mathrm{mg}, 65 \%$.

Appearance: dark amber oil.

$\boldsymbol{R}_{\mathbf{f}}=0.25$ (1:1 ethyl acetate:hexanes).

${ }^{1} \mathrm{H}$ NMR $\left(500 \mathrm{MHz}, \mathrm{CDCl}_{3}\right) \delta 8.69(\mathrm{br} \mathrm{s}, 1 \mathrm{H}), 7.72(\mathrm{q}, J=8.3 \mathrm{~Hz}, 2 \mathrm{H}), 7.43(\mathrm{~d}, J=7.5 \mathrm{~Hz}, 2 \mathrm{H}), 7.35-7.28(\mathrm{~m}$, $5 \mathrm{H}), 7.24(\mathrm{t}, J=7.4 \mathrm{~Hz}, 1 \mathrm{H}) 6.95(\mathrm{~d}, J=16.6 \mathrm{~Hz}, 1 \mathrm{H}), 6.79-6.68(\mathrm{~m}, 3 \mathrm{H}), 5.93(\mathrm{q}, J=1.4 \mathrm{~Hz}, 2 \mathrm{H}), 5.04(\mathrm{~d}, J=$ $15.0 \mathrm{~Hz}, 1 \mathrm{H}), 4.16(\mathrm{t}, J=3.0 \mathrm{~Hz}, 1 \mathrm{H}), 4.07-3.97(\mathrm{~m}, 1 \mathrm{H}), 3.94-3.80(\mathrm{~m}, 2 \mathrm{H}), 2.40(\mathrm{~s}, 3 \mathrm{H}), 1.94-1.77(\mathrm{~m}, 2 \mathrm{H})$, $1.73-1.36(\mathrm{~m}, 2 \mathrm{H})$ 0.98-0.90 (m, 2H).

${ }^{13} \mathrm{C} \mathrm{NMR}\left(176 \mathrm{MHz}, \mathrm{CDCl}_{3}\right) \delta 167.3,148.0,147.2,144.7,144.6,136.9,132.7,130.3,129.8(2 \mathrm{C}), 128.6$ (2C), $127.9,127.7$ (2C), 126.4 (2C), 121.4, 117.3, 108.5, 108.2, 101.1, 100.8, 70.0, 56.1, 44.0, 33.4, 28.4, 21.5, 19.3, 17.2.

IR (Diamond-ATR, neat) $\tilde{\mathbf{v}}_{\text {max }}:$ 3027, 2926, 1651, 1491, 1442, 1394, 1353, 1241, 1174, 1036, 909, 727.

HRMS (ESI) m/z: [M+H] $]^{+}$Calcd for $\mathrm{C}_{31} \mathrm{H}_{32} \mathrm{NO}_{7} \mathrm{~S}$ 562.1894; Found 562.1901. 
<smiles>O=C1C(O)/C(=C\c2ccccc2)C(CCCCO)N1Cc1ccc2c(c1)OCO2</smiles>

Solvent: $\mathrm{CDCl}_{3}$

Frequency: $500 \mathrm{MHz}$

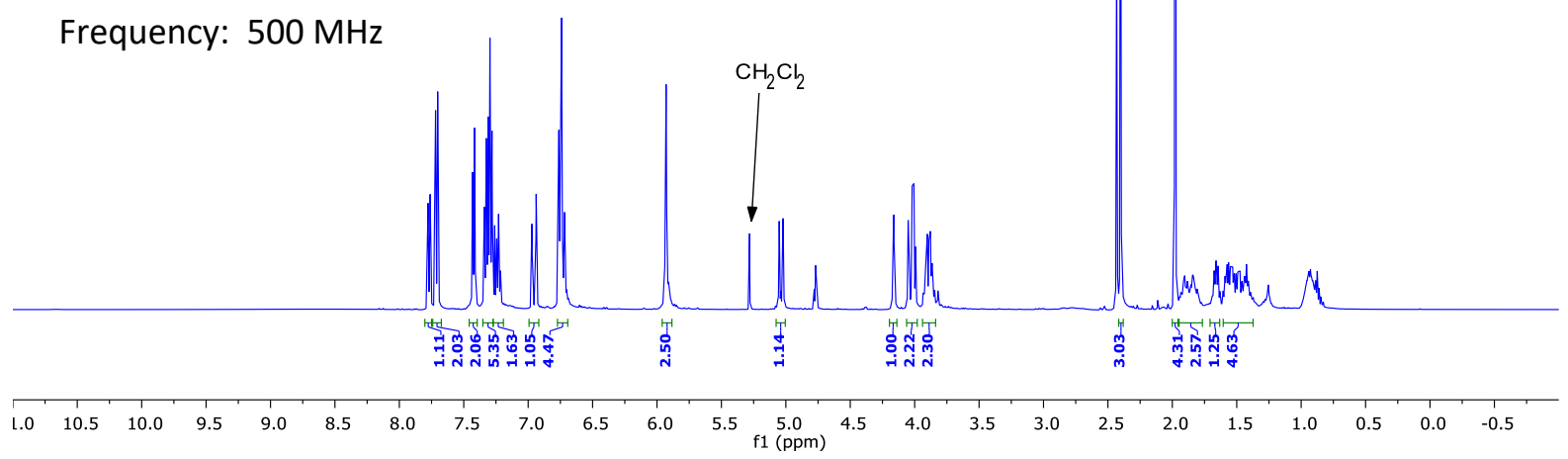

Feb02-2020-NM-20-068.19.fid

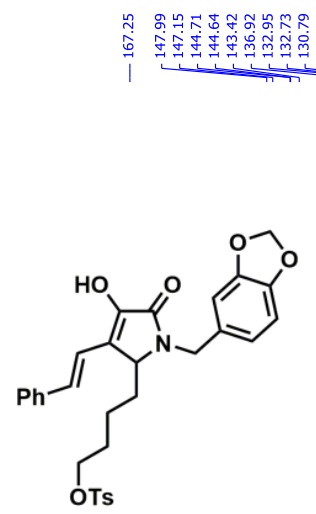

Solvent: $\mathrm{CDCl}_{3}$

Frequency: $176 \mathrm{MHz}$

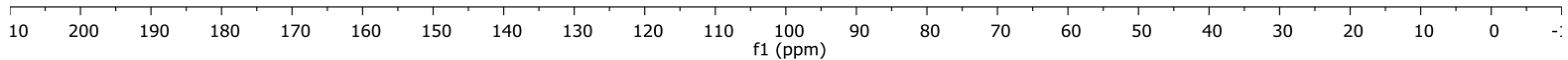


<smiles>O=C1C(O)=C(/C=C/c2ccccc2)C(CCCC[S+](F)(F)F)N1Cc1cccs1</smiles>

Yield: $60.6 \mathrm{mg}, 49 \%$.

Appearance: pale yellow grease.

$\boldsymbol{R}_{\mathbf{f}}=0.25$ (1:1 ethyl acetate:hexanes).

${ }^{1} \mathrm{H}$ NMR $\left(500 \mathrm{MHz}, \mathrm{CDCl}_{3}\right) \delta 8.08(\mathrm{br} \mathrm{s}, 1 \mathrm{H}), 7.72(\mathrm{~d}, J=8.3 \mathrm{~Hz}, 2 \mathrm{H}), 7.44(\mathrm{~d}, J=7.4 \mathrm{~Hz}, 2 \mathrm{H}), 7.35-7.26(\mathrm{~m}$, $5 \mathrm{H}), 7.26-7.22(\mathrm{~m}, 1 \mathrm{H}), 7.01(\mathrm{~d}, J=3.1 \mathrm{~Hz}, 1 \mathrm{H}), 6.96(\mathrm{dd}, J=5.5,2.1 \mathrm{~Hz}, 1 \mathrm{H}), 6.92(\mathrm{~d}, J=16.7 \mathrm{~Hz}, 1 \mathrm{H}), 6.77$ $(\mathrm{d}, J=16.5 \mathrm{~Hz}, 1 \mathrm{H}), 5.20(\mathrm{~d}, J=15.7 \mathrm{~Hz}, 1 \mathrm{H}), 4.39(\mathrm{~d}, J=15.7 \mathrm{~Hz}, 1 \mathrm{H}), 4.27(\mathrm{t}, J=3.4 \mathrm{~Hz}, 1 \mathrm{H}), 3.89$ (qt, $J=$ 10.0, 6.5 Hz, 2H), $2.41(\mathrm{~s}, 3 \mathrm{H}), 2.01-1.83(\mathrm{~m}, 2 \mathrm{H}), 1.62-1.46(\mathrm{~m}, 2 \mathrm{H}), 1.04-0.83(\mathrm{~m}, 2 \mathrm{H})$.

${ }^{13} \mathrm{C}$ NMR $\left(176 \mathrm{MHz}, \mathrm{CDCl}_{3}\right) \delta 167.0,144.8,143.1,138.8,136.9,132.8,131.2,129.8(2 \mathrm{C}), 128.7(2 \mathrm{C}), 128.0$, $127.8(2 \mathrm{C}), 127.0,126.9,126.5$ (2C), 125.8, 121.7, 117.2, 70.0, 56.4, 38.9, 28.5, 28.5, 21.6, 17.4.

IR (Diamond-ATR, neat) $\tilde{\mathbf{v}}_{\text {max }}:$ 3056, 2926, 2863, 1655, 1446, 1349, 1267, 1170, 936, 731, 693, 660.

HRMS (ESI) m/z: [M+H] $]^{+}$Calcd for $\mathrm{C}_{28} \mathrm{H}_{30} \mathrm{NO}_{5} \mathrm{~S}_{2}$ 524.1560; Found 524.1573. 


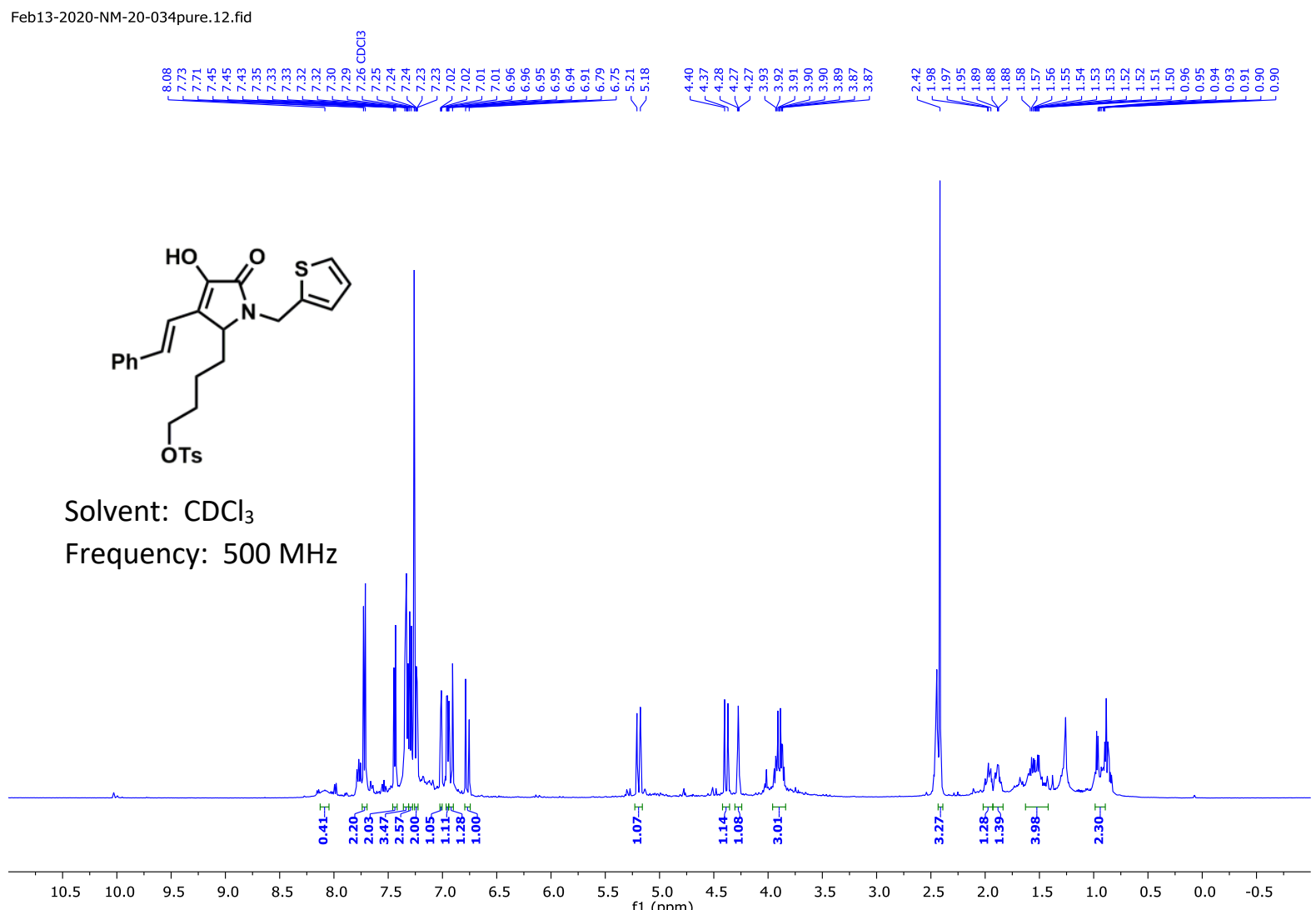

NM-20-034-C13.16.fid
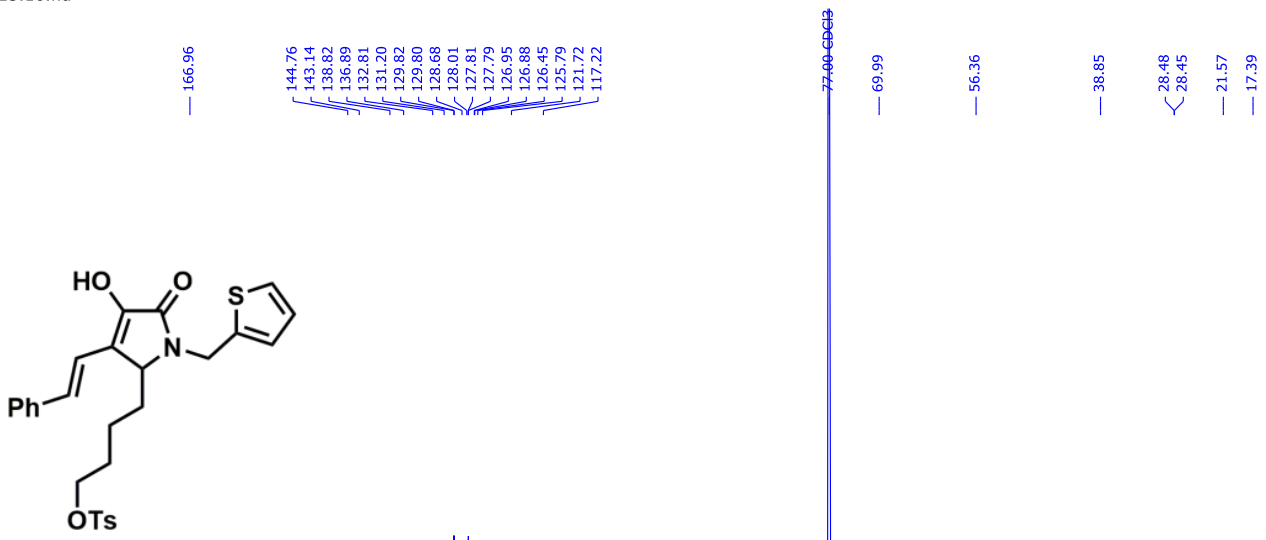

Solvent: $\mathrm{CDCl}_{3}$

Frequency: $176 \mathrm{MHz}$

\begin{tabular}{lllllllllllllllllllllllll}
\hline 10 & 200 & 190 & 180 & 170 & 160 & 150 & 140 & 130 & 120 & 110 & $\begin{array}{c}100 \\
\mathrm{f} 1(\mathrm{ppm})\end{array}$ & 90 & 80 & 70 & 60 & 50 & 40 & 30 & 20 & 10 & 0 & $-\therefore$
\end{tabular} 

(4g).<smiles>C=CCN1C(=O)C(O)=C(/C=C/c2ccccc2)C1CCCCO[S-]</smiles>

Yield: $73.9 \mathrm{mg}, 67 \%$.

Appearance: pale yellow oil.

$\boldsymbol{R}_{\mathrm{f}}=0.18$ (1:1 ethyl acetate:hexanes).

${ }^{1} \mathrm{H}$ NMR $\left(500 \mathrm{MHz}, \mathrm{CDCl}_{3}\right) \delta 8.37(\mathrm{br} \mathrm{s}, 1 \mathrm{H}), 7.71(\mathrm{~d}, J=8.2 \mathrm{~Hz}, 2 \mathrm{H}), 7.46(\mathrm{~d}, J=7.6 \mathrm{~Hz}, 2 \mathrm{H}), 7.37-7.31(\mathrm{~m}$, $3 \mathrm{H}), 7.29(\mathrm{~d}, J=8.2 \mathrm{~Hz}, 2 \mathrm{H}), 7.27-7.24(\mathrm{~m}, 1 \mathrm{H}), 6.97(\mathrm{~d}, J=16.5 \mathrm{~Hz}, 1 \mathrm{H}), 6.79(\mathrm{~d}, J=16.6 \mathrm{~Hz}, 1 \mathrm{H}), 5.78$ (dddd, $J=17.4,10.2,7.5,4.9 \mathrm{~Hz}, 1 \mathrm{H}), 5.27-5.20(\mathrm{~m}, 2 \mathrm{H}), 4.52(\mathrm{ddt}, J=15.5,4.9,1.7 \mathrm{~Hz}, 1 \mathrm{H}), 4.33(\mathrm{t}, J=3.6$ $\mathrm{Hz}, 1 \mathrm{H}), 3.98-3.87(\mathrm{~m}, 2 \mathrm{H}), 3.60(\mathrm{dd}, J=15.6,7.5 \mathrm{~Hz}, 1 \mathrm{H}), 2.41(\mathrm{~s}, 3 \mathrm{H}), 1.99-1.80(\mathrm{~m}, 2 \mathrm{H}), 1.59(\mathrm{dtd}, J=$ $15.2,6.6,2.0 \mathrm{~Hz}, 2 \mathrm{H}), 1.04-0.89(\mathrm{~m}, 2 \mathrm{H})$.

${ }^{13} \mathrm{CNMR}\left(176 \mathrm{MHz}, \mathrm{CDCl}_{3}\right) \delta 167.0,144.7,143.3,137.0,132.8,132.7,131.0,129.8(2 \mathrm{C}), 128.7(2 \mathrm{C}), 128.0$, $127.8(2 \mathrm{C}), 126.5(2 \mathrm{C}), 121.3,118.4,117.3,70.0,56.4,43.1,28.5,28.3,21.6,17.3$.

IR (Diamond-ATR, neat) $\tilde{v}_{\text {max }}:$ 3027, 2928, 1653, 1595, 1448, 1353, 1172, 932 751, 662.

HRMS (ESI) m/z: [M+H] ${ }^{+}$Calcd for $\mathrm{C}_{26} \mathrm{H}_{30} \mathrm{NO}_{5} \mathrm{~S}$ 468.1839; Found 468.1849. 


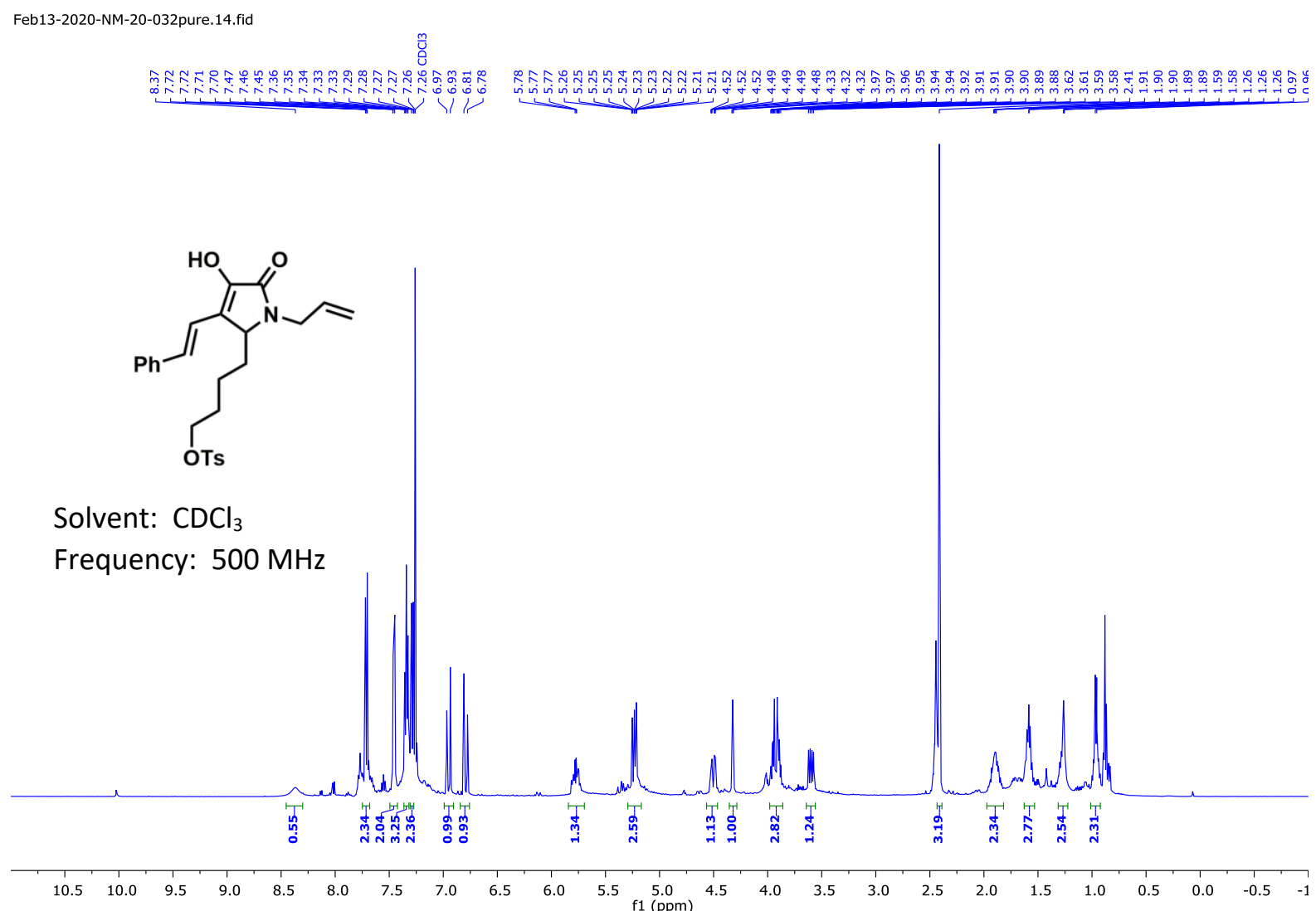

NM-20-032-C13.14.fid
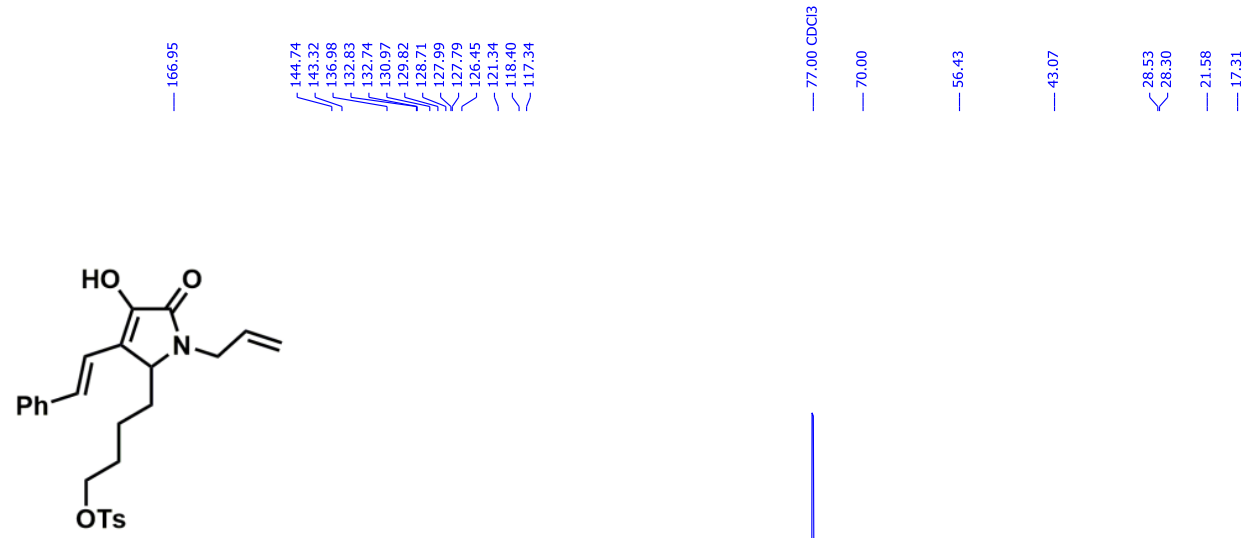

Solvent: $\mathrm{CDCl}_{3}$

Frequency: $176 \mathrm{MHz}$

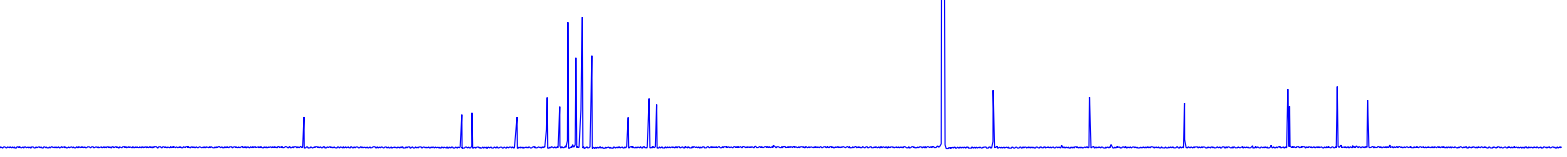

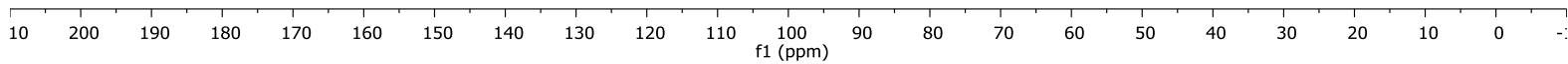




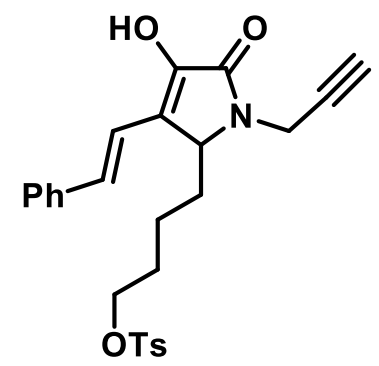

Yield: $74.0 \mathrm{mg}, 67 \%$.

Appearance: pale yellow oil.

$\boldsymbol{R}_{\mathbf{f}}=0.19$ (1:1 ethyl acetate:hexanes).

${ }^{1} \mathrm{H}$ NMR $\left(500 \mathrm{MHz}, \mathrm{CDCl}_{3}\right) \delta 8.52(\mathrm{br} \mathrm{s}, 1 \mathrm{H}), 7.71(\mathrm{~d}, J=8.3 \mathrm{~Hz}, 2 \mathrm{H}), 7.47(\mathrm{~d}, J=7.4 \mathrm{~Hz}, 2 \mathrm{H}), 7.35(\mathrm{t}, J=7.3$ $\mathrm{Hz}, 2 \mathrm{H}), 7.30-7.26(\mathrm{~m}, 3 \mathrm{H}), 6.97(\mathrm{~d}, J=16.6 \mathrm{~Hz}, 1 \mathrm{H}), 6.85(\mathrm{~d}, J=16.6 \mathrm{~Hz}, 1 \mathrm{H}), 4.59(\mathrm{dd}, J=17.9,2.6 \mathrm{~Hz}, 1 \mathrm{H})$, $4.49(\mathrm{t}, J=3.6 \mathrm{~Hz}, 1 \mathrm{H}), 3.98-3.86(\mathrm{~m}, 3 \mathrm{H}), 2.41(\mathrm{~s}, 3 \mathrm{H}), 2.28(\mathrm{t}, J=2.5 \mathrm{~Hz}, 1 \mathrm{H}), 2.08-1.88(\mathrm{~m}, 2 \mathrm{H}), 1.65-$ $1.56(\mathrm{~m}, 2 \mathrm{H}), 1.07-0.95(\mathrm{~m}, 2 \mathrm{H})$.

${ }^{13} \mathrm{C} \mathrm{NMR}\left(176 \mathrm{MHz}, \mathrm{CDCl}_{3}\right) \delta 166.7,144.8,142.9,136.9,132.8,131.5,129.8$ (2C), 128.7 (2C), 128.1, 127.8 (2C), 126.5 (2C), 122.1, 117.1, 77.5, 72.6, 70.0, 56.9, 30.2, 28.6, 28.5, 21.6, 17.6.

IR (Diamond-ATR, neat) $\tilde{\mathbf{v}}_{\text {max }}: 3280,3056,2926,2866,1659,1446,1349,1267,1170,936,731$.

HRMS (ESI) m/z: [M+H] $]^{+}$Calcd for $\mathrm{C}_{26} \mathrm{H}_{28} \mathrm{NO}_{5} \mathrm{~S}$ 466.1683; Found 466.1691. 
Feb01-2020-NM-20-033.16.fid
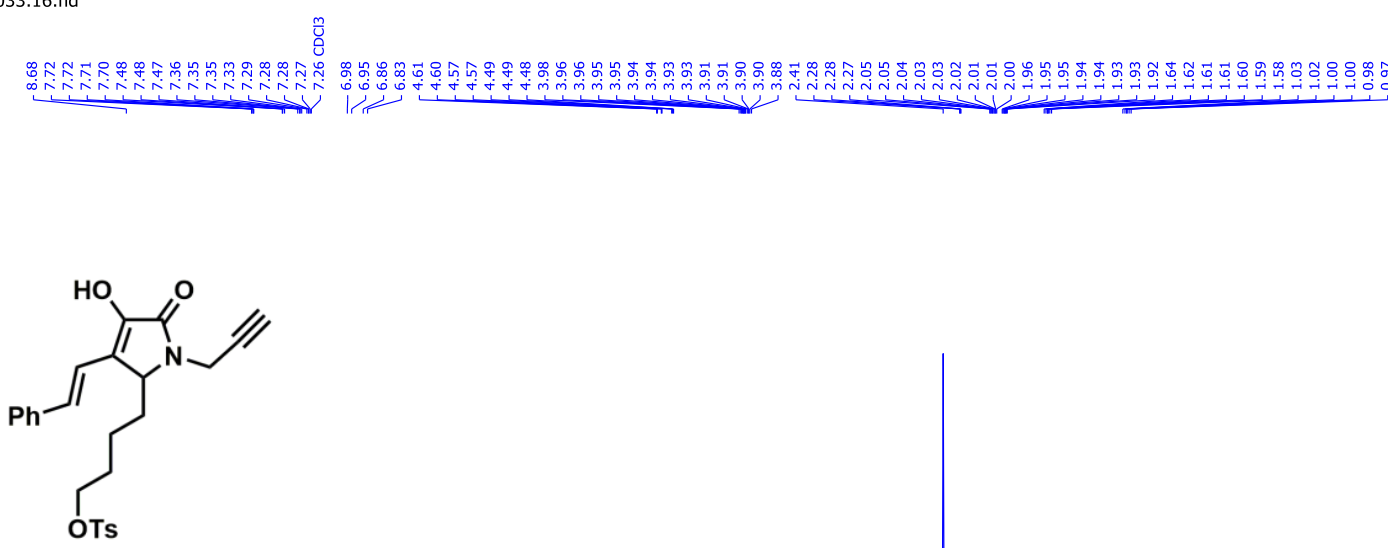

Solvent: $\mathrm{CDCl}_{3}$

Frequency: $500 \mathrm{MHz}$

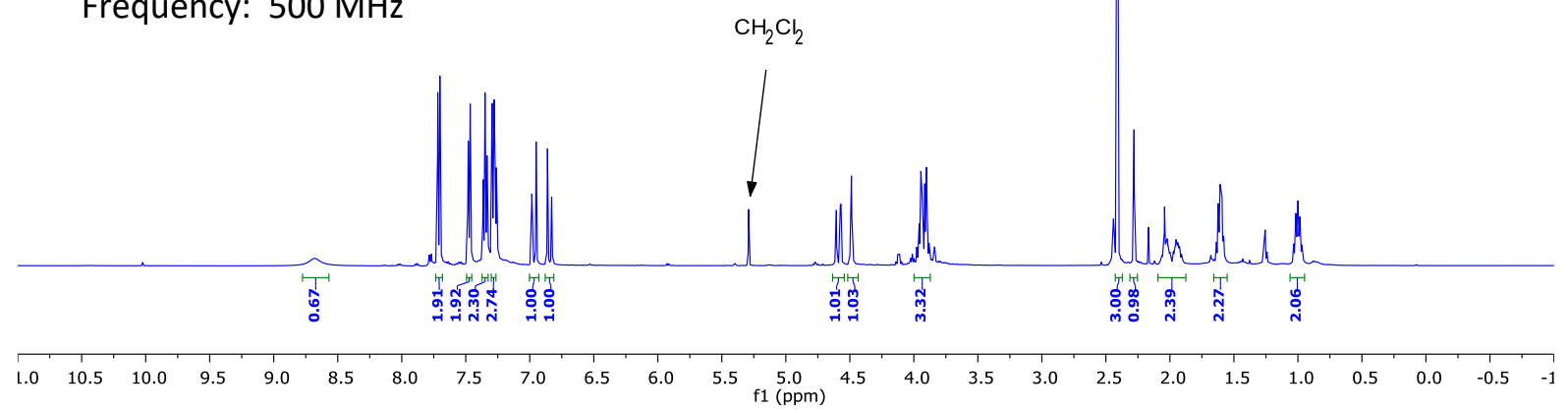

NM-20-033-C13.15.fid
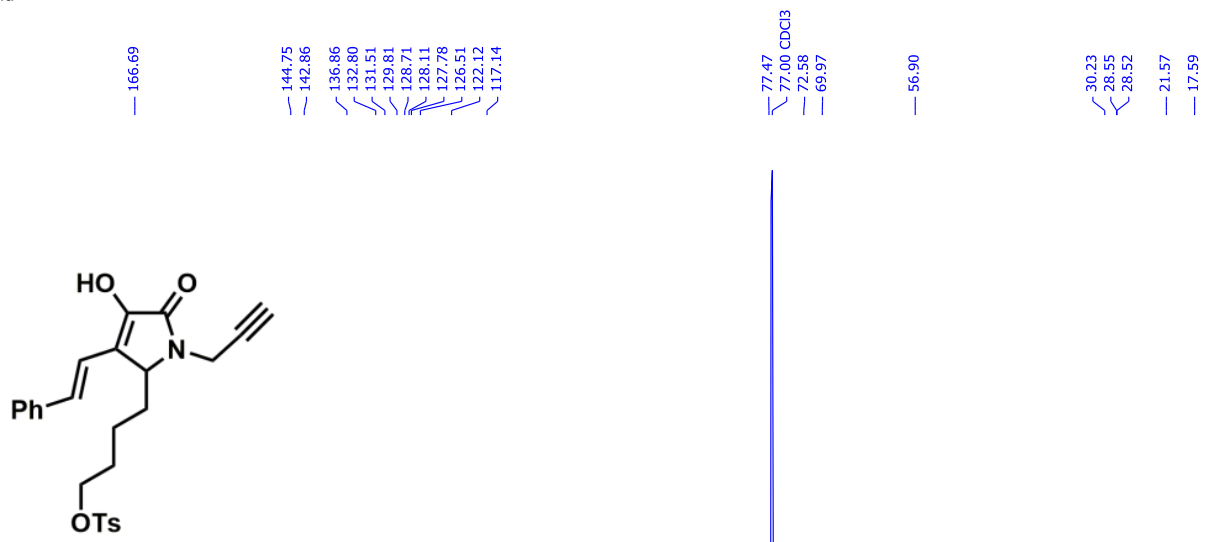

Solvent: $\mathrm{CDCl}_{3}$

Frequency: $176 \mathrm{MHz}$

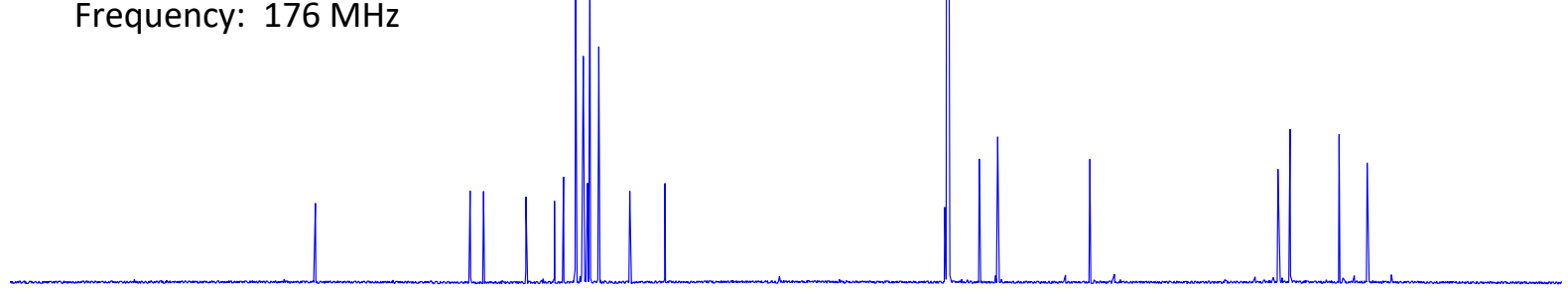

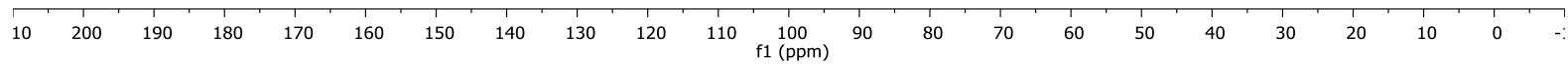


<smiles>CN1C(=O)C(O)=C(c2ccccc2)C1CCCC[O-]</smiles>

Yield: $66.9 \mathrm{mg}, 50 \%$.

Appearance: off-white oil.

$\boldsymbol{R}_{\mathrm{f}}=0.12$ (1:1 ethyl acetate:hexanes).

${ }^{1} \mathrm{H}$ NMR $\left(500 \mathrm{MHz}, \mathrm{CDCl}_{3}\right) \delta 7.98(\mathrm{br} \mathrm{s}, 1 \mathrm{H}), 7.70(\mathrm{~d}, J=8.3 \mathrm{~Hz}, 2 \mathrm{H}), 7.58(\mathrm{~d}, J=7.3 \mathrm{~Hz}, 2 \mathrm{H}), 7.38(\mathrm{t}, J=7.8$ $\mathrm{Hz}, 2 \mathrm{H}), 7.31(\mathrm{~d}, J=8.0 \mathrm{~Hz}, 2 \mathrm{H}), 7.29-7.23(\mathrm{~m}, 1 \mathrm{H}), 4.43(\mathrm{t}, J=3.7 \mathrm{~Hz}, 1 \mathrm{H}), 3.83(\mathrm{qt}, J=9.9,6.5 \mathrm{~Hz}, 2 \mathrm{H}), 3.02$ (s, 3H), $2.42(\mathrm{~s}, 3 \mathrm{H}), 1.96-1.76(\mathrm{~m}, 2 \mathrm{H}), 1.46(\mathrm{t}, J=7.2 \mathrm{~Hz}, 2 \mathrm{H}), 0.98-0.74(\mathrm{~m}, 2 \mathrm{H})$.

${ }^{13} \mathrm{C}$ NMR $\left(176 \mathrm{MHz}, \mathrm{CDCl}_{3}\right) \delta 167.2,144.8,142.4,132.9,131.4,129.8(2 \mathrm{C}), 128.6(2 \mathrm{C}), 127.8(2 \mathrm{C}), 127.6$, $127.1(2 \mathrm{C}), 120.5,69.9,58.9,28.5,28.3,27.7,21.6,17.3$.

IR (Diamond-ATR, neat) $\tilde{\text { vax}}_{\text {max }}$ 3127, 2911, 2855, 1659, 1301, 1155, 954, 783.

HRMS (ESI) m/z: [M+H] $]^{+}$Calcd for $\mathrm{C}_{22} \mathrm{H}_{26} \mathrm{NO}_{5} \mathrm{~S}$ 416.1526; Found 416.1531.

[15] Following addition of substrates, $200 \mu \mathrm{L}$ of anhydrous methanol and triethylamine (1.1 equiv, $0.37 \mathrm{mmol}, 52.2 \mu \mathrm{L}$ ) were subsequently added to the reaction mixture. Reaction scale was 0.34 mmol based on limiting reagent $1 \mathrm{a}$. 

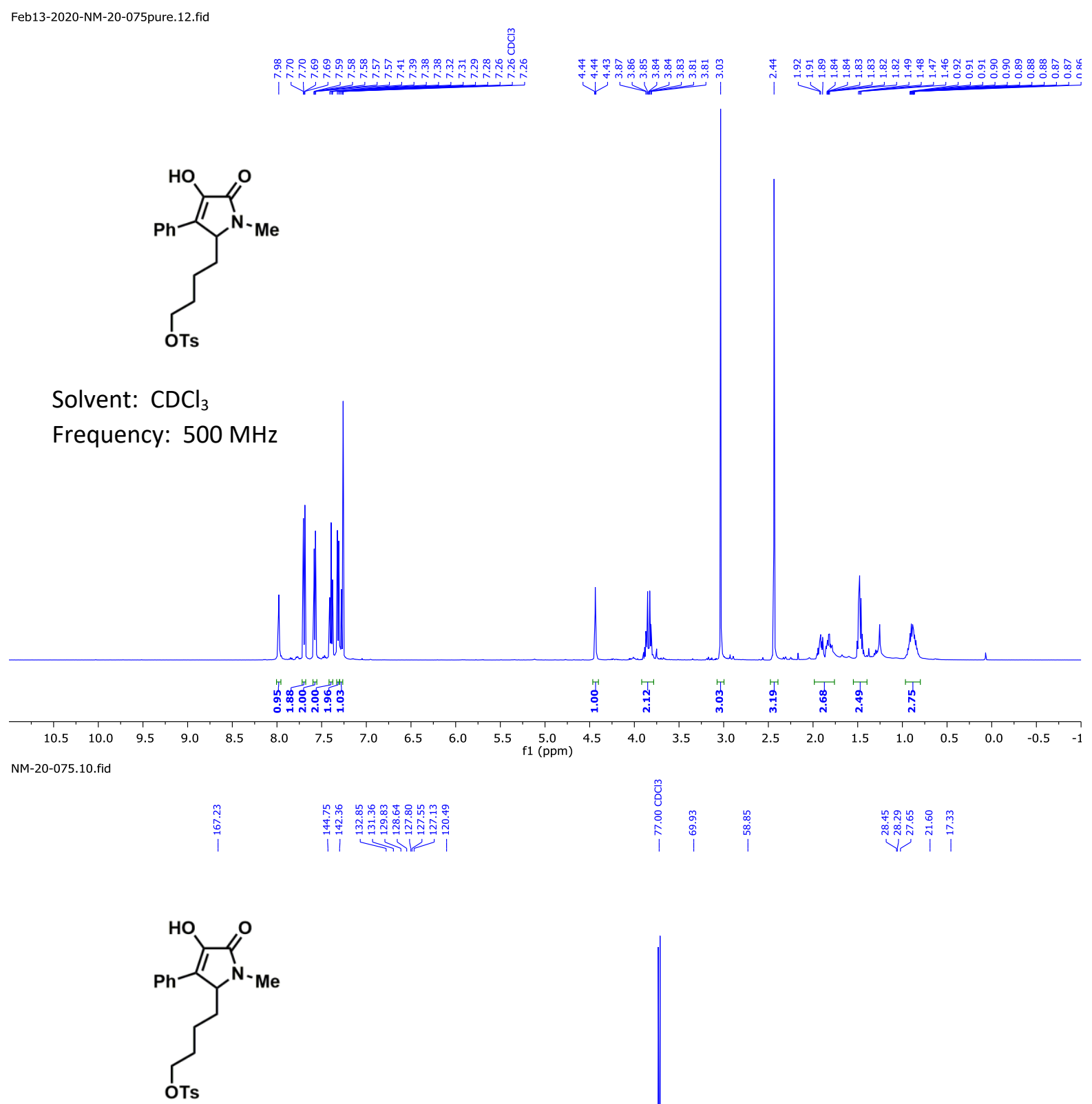

Solvent: $\mathrm{CDCl}_{3}$

Frequency: $176 \mathrm{MHz}$

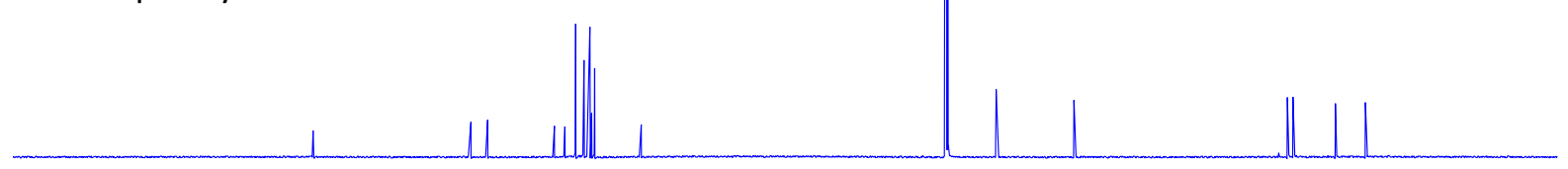

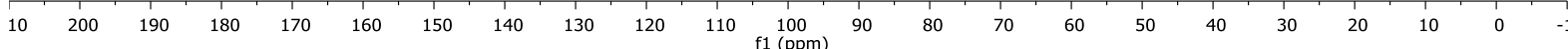




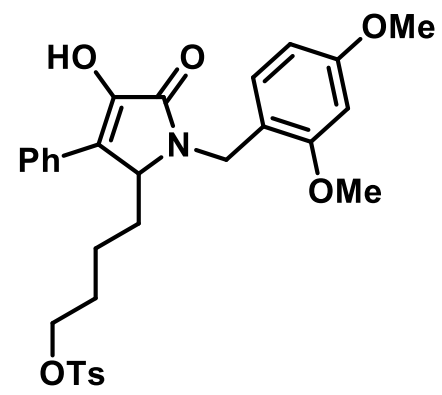

Yield: $146 \mathrm{mg}, 49 \% .1 .69 \mathrm{~g}, 61 \%{ }^{16}$

Appearance: white solid.

$\boldsymbol{R}_{\mathbf{f}}=0.23$ (1:1 ethyl acetate:hexanes).

${ }^{1} \mathrm{H}$ NMR $\left(500 \mathrm{MHz}, \mathrm{CDCl}_{3}\right) \delta 7.70(\mathrm{~d}, J=8.2 \mathrm{~Hz}, 2 \mathrm{H}), 7.52(\mathrm{~d}, J=7.7 \mathrm{~Hz}, 2 \mathrm{H}), 7.36(\mathrm{t}, J=7.6 \mathrm{~Hz}, 2 \mathrm{H}), 7.34-$ $7.30(\mathrm{~m}, 3 \mathrm{H}), 7.17(\mathrm{~d}, J=8.2 \mathrm{~Hz}, 1 \mathrm{H}), 6.49-6.42(\mathrm{~m}, 2 \mathrm{H}), 4.95(\mathrm{~d}, J=15.0 \mathrm{~Hz}, 1 \mathrm{H}), 4.36(\mathrm{t}, J=3.6 \mathrm{~Hz}, 1 \mathrm{H})$, $4.26(\mathrm{~d}, J=15.0 \mathrm{~Hz}, 1 \mathrm{H}), 3.83(\mathrm{~s}, 3 \mathrm{H}), 3.82-3.78(\mathrm{~m}, 5 \mathrm{H}), 2.44(\mathrm{~s}, 3 \mathrm{H}), 2.01-1.89(\mathrm{~m}, 1 \mathrm{H}), 1.78-1.69(\mathrm{~m}, 1 \mathrm{H})$, 1.41 (ddd, $J=8.8,6.6,4.9 \mathrm{~Hz}, 2 \mathrm{H}), 0.98-0.76(\mathrm{~m}, 2 \mathrm{H})$, (did not observe $\mathrm{OH}$ signal).

${ }^{13} \mathrm{C} \mathrm{NMR}\left(176 \mathrm{MHz}, \mathrm{CDCl}_{3}\right) \delta 167.0,160.6,158.3,144.7,141.8,132.9,131.4,130.9,129.8(2 \mathrm{C}), 128.6(2 \mathrm{C})$, 127.8 (2C), 127.5, 127.2 (2C), 120.4, 117.2, 104.5, 98.5, 70.1, 56.5, 55.4, 55.4, 38.4, 28.6, 27.9, 21.6, 17.3.

IR (Diamond-ATR, neat) $\tilde{V}_{\max }: 3086,2926,1659,1610,1502,1353,1170,1032,928$.

HRMS (ESI) m/z: [M+H] $]^{+}$Calcd for $\mathrm{C}_{30} \mathrm{H}_{34} \mathrm{NO}_{7} \mathrm{~S} 552.2051$; Found 552.2047.

[16] Synthesis of $\mathbf{4 j}$ at a $5.16 \mathrm{mmol}$ scale. To a solution of $\mathbf{1 b}$ ( $920 \mathrm{mg}, 5.16 \mathrm{mmole}, 1$ equiv) and $\mathbf{2 a}$ (1.5 g, $5.68 \mathrm{mmol}, 1.1$ equiv) in $6 \mathrm{~mL}$ of anhydrous dichloromethane was added a solution of $3 a$ ( $870 \mu \mathrm{L}, 5.68 \mathrm{mmol}, 1.1$ equiv) in $4 \mathrm{~mL}$ of anhydrous dichloromethane via addition funnel at 0 ${ }^{\circ} \mathrm{C}$ over a duration of $5 \mathrm{~min}$. Reaction was then stirred for an additional $35 \mathrm{~min}$ at this temperature. Reaction was allowed to stir to room temperature for an additional hour when full consumption of $\mathbf{1 b}$ was observed by TLC. Reaction was concentrated to volume by rotoevaporation and immediately purified by flash column chromatography using a gradient from hexanes to 1:1 ethyl acetate:hexanes mixture yielding pyrrole substrate $4 \mathbf{j}$ ( $1.6909 \mathrm{~g}, 61 \%)$. 

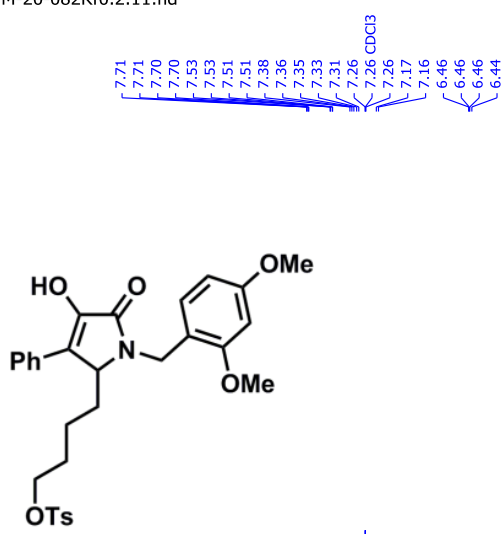

Solvent: $\mathrm{CDCl}_{3}$

Frequency: $500 \mathrm{MHz}$

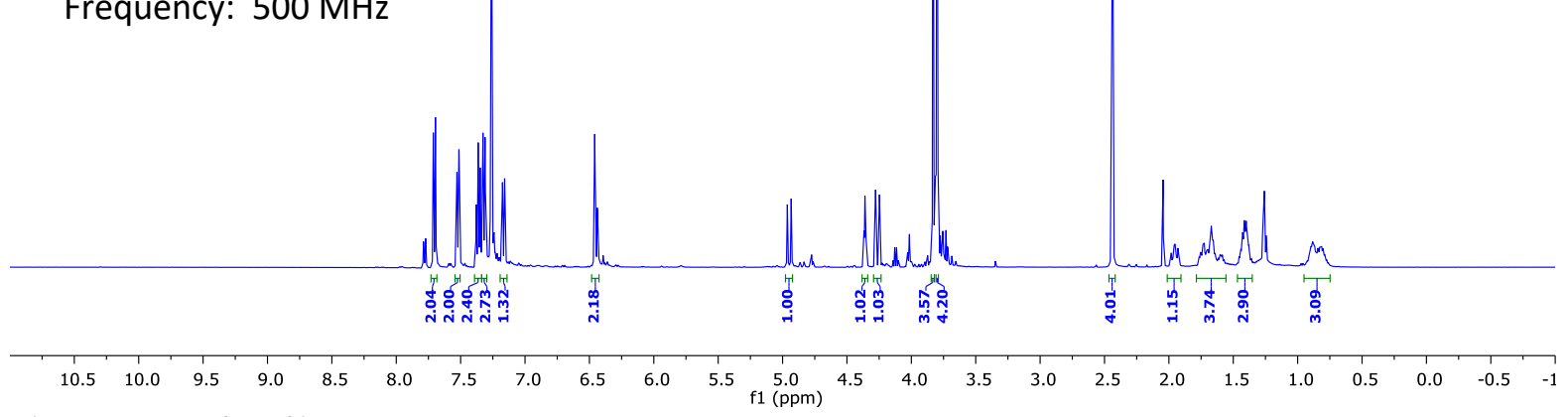
Feb20-2020-NM-20-082Rfo.2.12.fid

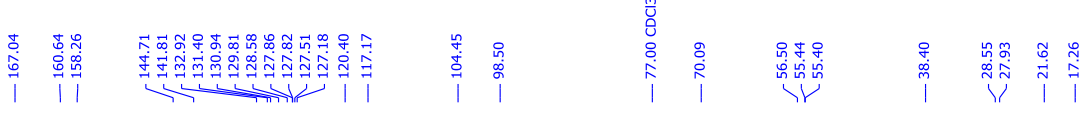

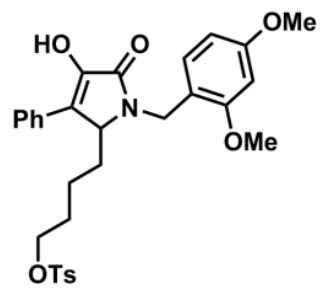

Solvent: $\mathrm{CDCl}_{3}$

Frequency: $176 \mathrm{MHz}$

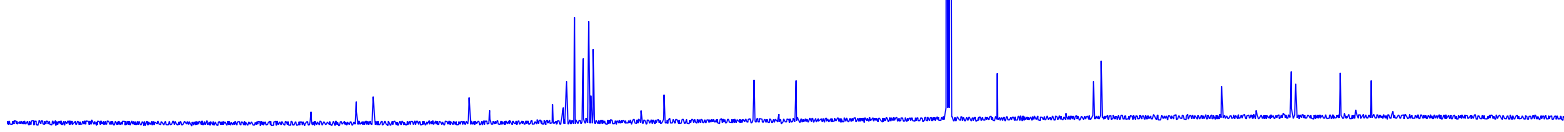

$\begin{array}{lllllllllllllllllllllll}10 & 200 & 190 & 180 & 170 & 160 & 150 & 140 & 130 & 120 & 110 & \begin{array}{c}100 \\ \mathrm{f} 1(\mathrm{ppm})\end{array} & 90 & 80 & 70 & 60 & 50 & 40 & 30 & 20 & 10 & 0 & -:\end{array}$ 

methylbenzenesulfonate (4k).

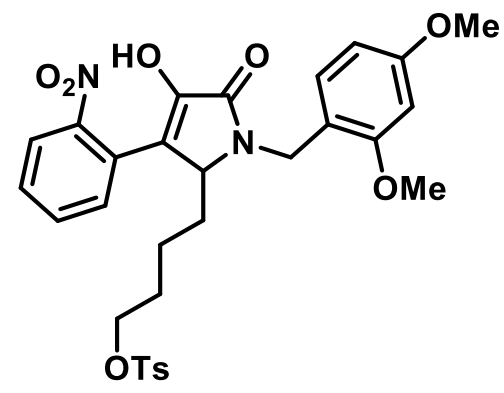

Yield: $106 \mathrm{mg}, 68 \%$.

Appearance: yellow solid.

$\boldsymbol{R}_{\mathbf{f}}=0.13$ (1:1 ethyl acetate:hexanes).

${ }^{1} \mathrm{H}$ NMR $\left(500 \mathrm{MHz}, \mathrm{CDCl}_{3}\right) \delta 7.88(\mathrm{dd}, J=8.2,1.3 \mathrm{~Hz}, 1 \mathrm{H}), 7.72(\mathrm{~d}, \mathrm{~J}=8.3 \mathrm{~Hz}, 2 \mathrm{H}), 7.61$ (br s, $\left.1 \mathrm{H}\right), 7.58$ (td, $J=7.6,1.3 \mathrm{~Hz}, 1 \mathrm{H}), 7.43(\mathrm{td}, J=7.9,1.3 \mathrm{~Hz}, 1 \mathrm{H}), 7.36-7.31(\mathrm{~m}, 3 \mathrm{H}), 7.18(\mathrm{~d}, J=8.5 \mathrm{~Hz}, 1 \mathrm{H}), 6.48-6.44(\mathrm{~d}, J$ $=7.4 \mathrm{~Hz}, 2 \mathrm{H}), 4.87(\mathrm{~d}, J=14.9 \mathrm{~Hz}, 1 \mathrm{H}), 4.35(\mathrm{dd}, J=5.0,2.9 \mathrm{~Hz}, 1 \mathrm{H}), 4.24(\mathrm{~d}, J=14.9 \mathrm{~Hz}, 1 \mathrm{H}), 3.86-3.70(\mathrm{~m}$, $8 \mathrm{H}), 2.42(\mathrm{~s}, 3 \mathrm{H}), 1.89-1.78(\mathrm{~m}, 1 \mathrm{H}), 1.48-1.35(\mathrm{~m}, 3 \mathrm{H}), 1.16-1.04(\mathrm{~m}, 1 \mathrm{H}), 0.98-0.87(\mathrm{~m}, 1 \mathrm{H})$.

${ }^{13} \mathrm{C}$ NMR $\left(176 \mathrm{MHz}, \mathrm{CDCl}_{3}\right) \delta 166.4,160.6,158.2,149.1,144.8,144.4,132.8,132.6,131.1,130.7,129.8$ (2C), 128.4, 127.7 (2C), 126.2, 124.9, 117.5, 116.9, 104.5, 98.4, 70.0, 57.3, 55.3, 55.3, 38.8, 28.6, 28.1, 21.5, 17.7.

IR (Diamond-ATR, neat) $\tilde{V}_{\max }:$ 3008, 2933, 2840, 1662, 1610, 1528, 1454, 1353, 1208, 1174, 1032, 753.

HRMS (ESI) m/z: [M+H] $]^{+}$Calcd for $\mathrm{C}_{30} \mathrm{H}_{33} \mathrm{~N}_{2} \mathrm{O}_{9} \mathrm{~S}$ 597.1901; Found 597.1912. 

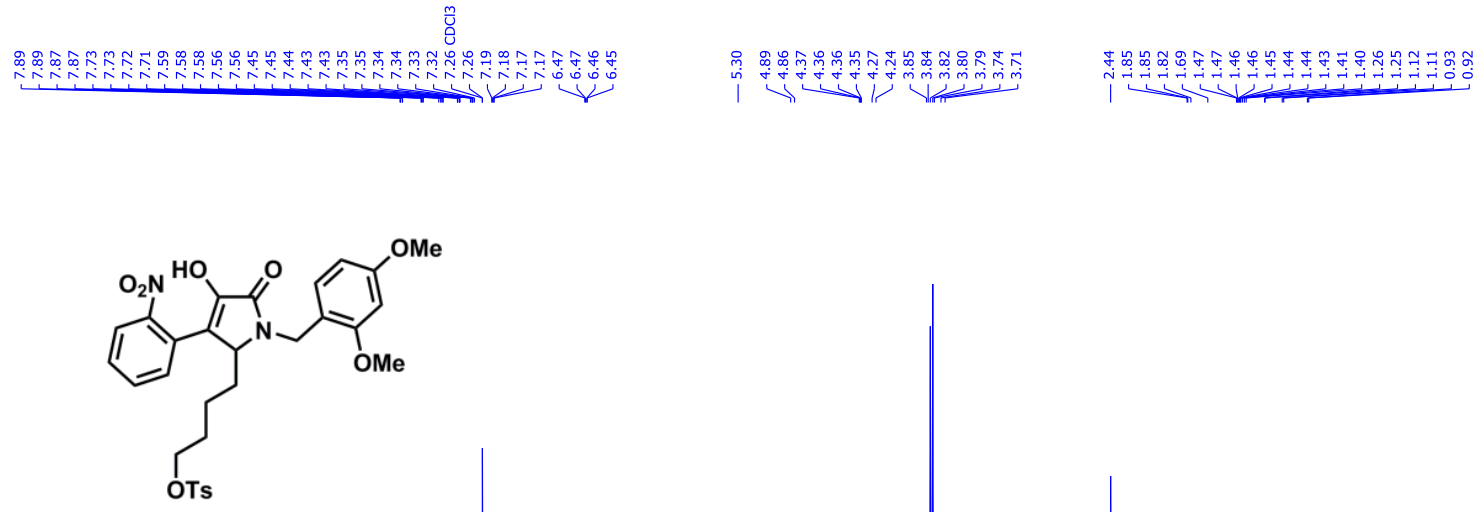

Solvent: $\mathrm{CDCl}_{3}$

Frequency: $500 \mathrm{MHz}$

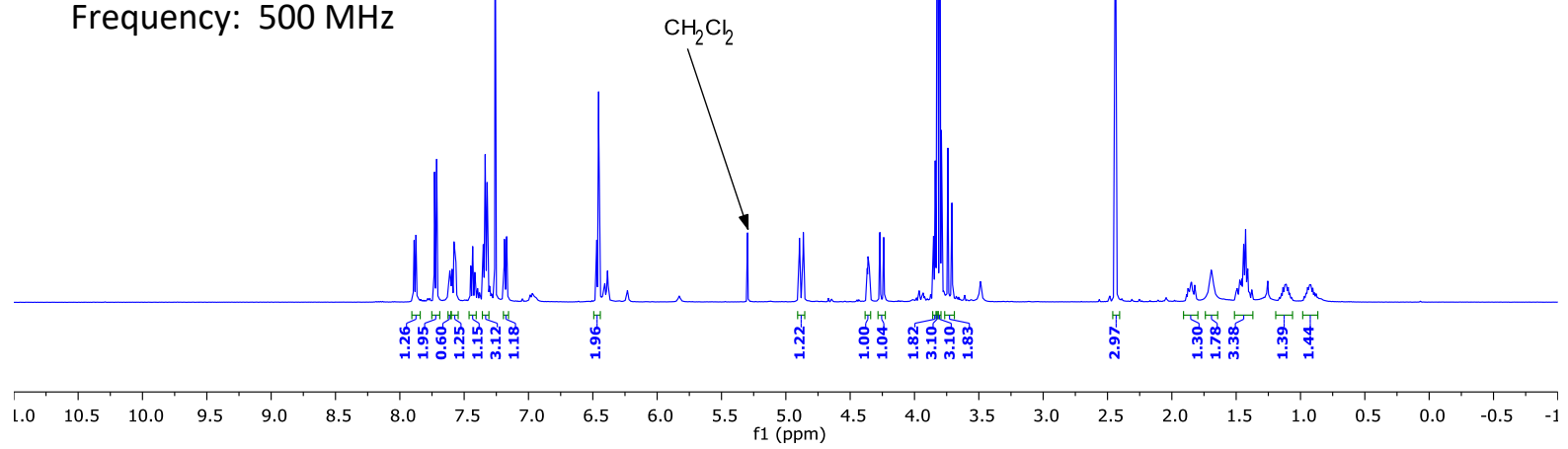
Feb02-2020-NM-20-062.16.fid
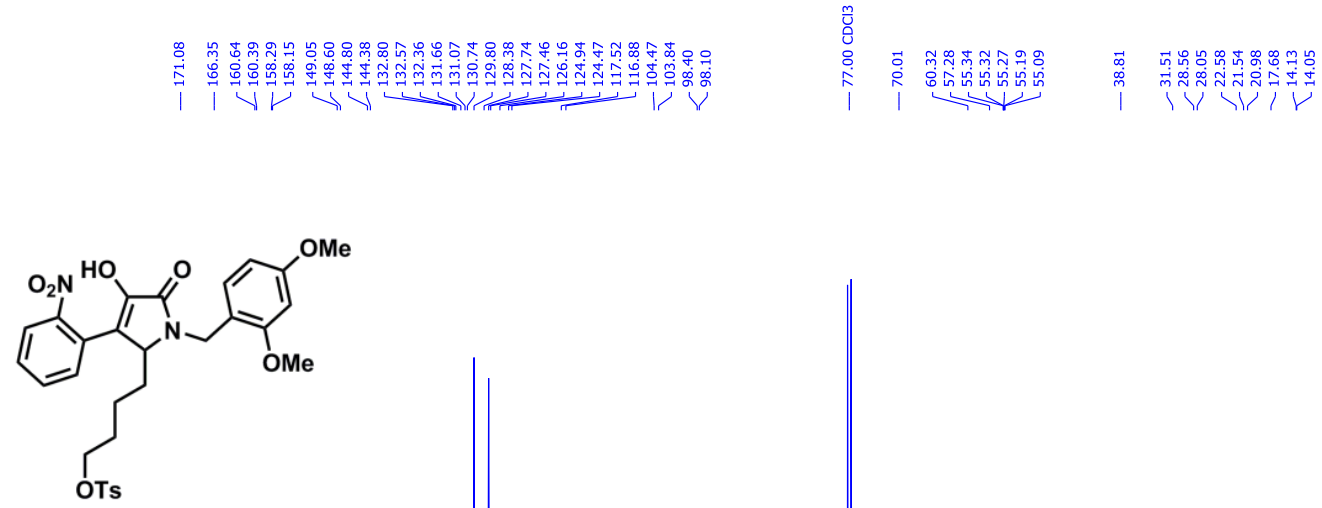

Solvent: $\mathrm{CDCl}_{3}$

Frequency: $176 \mathrm{MHz}$

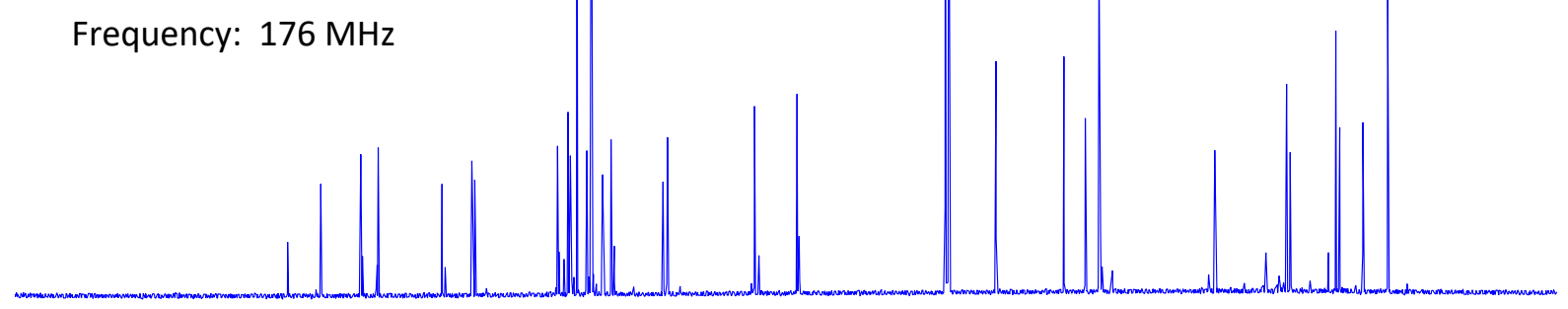

$10 \quad 200$

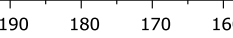

$150 \quad 140$

130

f1 100

$80 \quad 70 \quad 60 \quad 50$ 
(3-(Benzo[d][1,3]dioxol-5-yl)-1-(2,4-dimethoxybenzyl)-4-hydroxy-5-oxo-2,5-dihydro-1H-pyrrol-2yl)butyl 4-methylbenzenesulfonate (4I).<smiles>COc1ccc(CN2C(=O)C(O)=C(c3ccc4c(c3)OCO4)C2CCCC[SeH2])c(OC)c1</smiles>

Yield: $49.2 \mathrm{mg}, 38 \%$.

Appearance: Pale yellow oil.

$\boldsymbol{R}_{\mathrm{f}}=0.21$ (1:1 ethyl acetate:hexanes).

${ }^{1} \mathrm{H}$ NMR $\left(500 \mathrm{MHz}, \mathrm{CDCl}_{3}\right) \delta 7.71(\mathrm{~d}, J=8.3 \mathrm{~Hz}, 2 \mathrm{H}), 7.33(\mathrm{~d}, J=8.0 \mathrm{~Hz}, 2 \mathrm{H}), 7.16(\mathrm{~d}, J=8.1 \mathrm{~Hz}, 1 \mathrm{H}), 7.14(\mathrm{~d}$, $J=1.7 \mathrm{~Hz}, 1 \mathrm{H}), 6.93(\mathrm{~d}, J=1.7 \mathrm{~Hz}, 1 \mathrm{H}), 6.80(\mathrm{~d}, J=8.1 \mathrm{~Hz}, 1 \mathrm{H}), 6.61(\mathrm{br} \mathrm{s}, 1 \mathrm{H}) 6.48-6.42(\mathrm{~m}, 2 \mathrm{H}), 5.96(\mathrm{~s}$, $2 \mathrm{H}), 4.93(\mathrm{~d}, J=15.0 \mathrm{~Hz}, 1 \mathrm{H}), 4.29-4.21(\mathrm{~m}, 2 \mathrm{H}), 3.85-3.78(\mathrm{~m}, 8 \mathrm{H}), 2.44(\mathrm{~s}, 3 \mathrm{H}), 2.00-1.88(\mathrm{~m}, 1 \mathrm{H}), 1.78-$ $1.55(\mathrm{~m}, 1 \mathrm{H}), 1.41(\mathrm{dq}, J=9.0,6.6 \mathrm{~Hz}, 2 \mathrm{H}), 0.91-0.76(\mathrm{~m}, 2 \mathrm{H})$.

${ }^{13} \mathrm{C} \mathrm{NMR}\left(126 \mathrm{MHz}, \mathrm{CDCl}_{3}\right) \delta 167.3,160.6,158.2,147.9,146.9,144.7,141.1,132.9,131.0,129.8(2 \mathrm{C}), 127.8$ (2C), 125.6, 120.9, 120.7, 117.2, 108.4, 107.8, 104.4, 101.1, 98.5, 70.1, 56.6, 55.4, 55.4, 38.3, 28.5, 28.0, 21.6, 17.2.

IR (Diamond-ATR, neat) $\tilde{V}_{\max }: 3060,2937,1659,1610,1498,1446,1237,1174,1032,928,813$.

HRMS (ESI) m/z: [M+H] $]^{+}$Calcd for $\mathrm{C}_{31} \mathrm{H}_{34} \mathrm{NO}_{9} \mathrm{~S}$ 596.1949; Found 596.1940. 


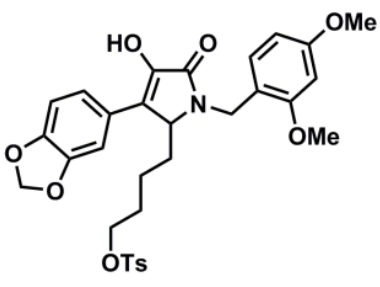

Solvent: $\mathrm{CDCl}_{3}$

Frequency: $500 \mathrm{MHz}$

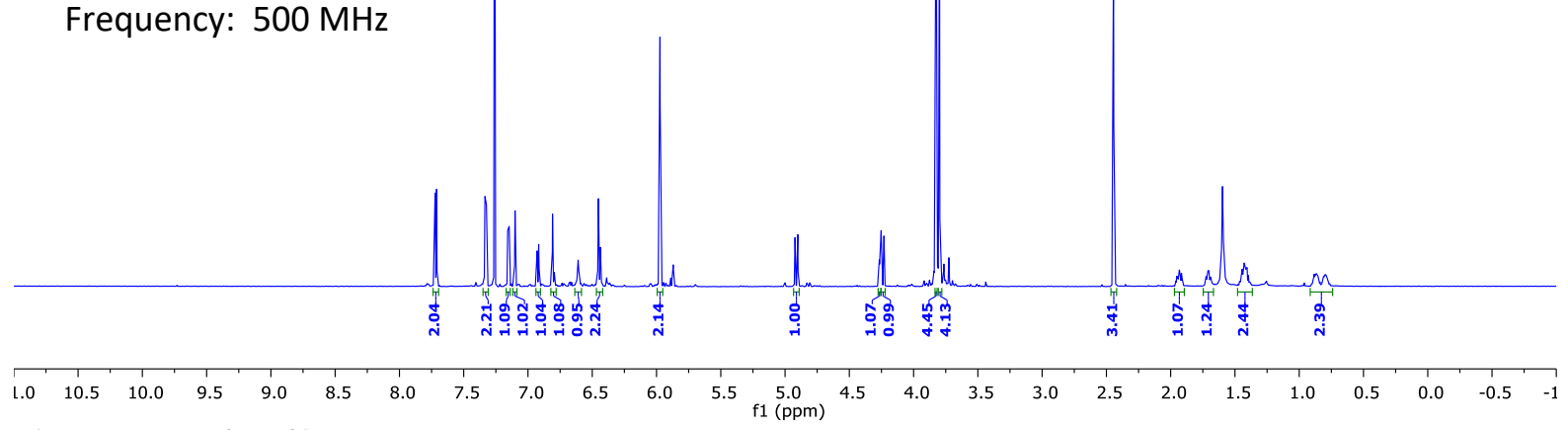
Feb19-2020-NM-20-085Rfo.2.21.fid
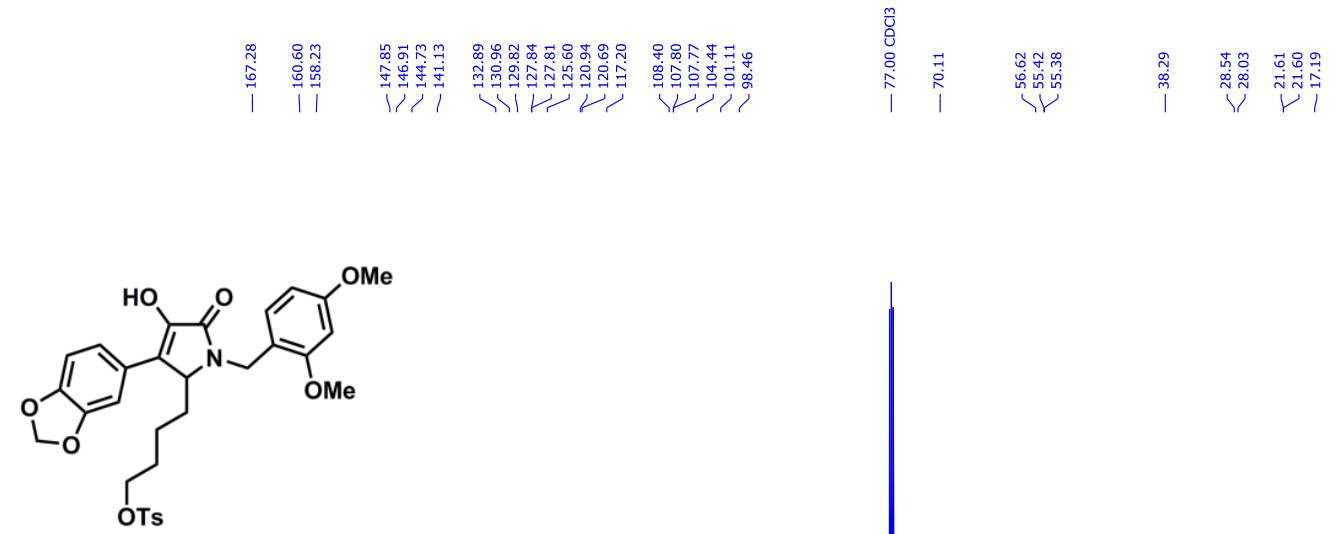

Solvent: $\mathrm{CDCl}_{3}$

Frequency: $126 \mathrm{MHz}$

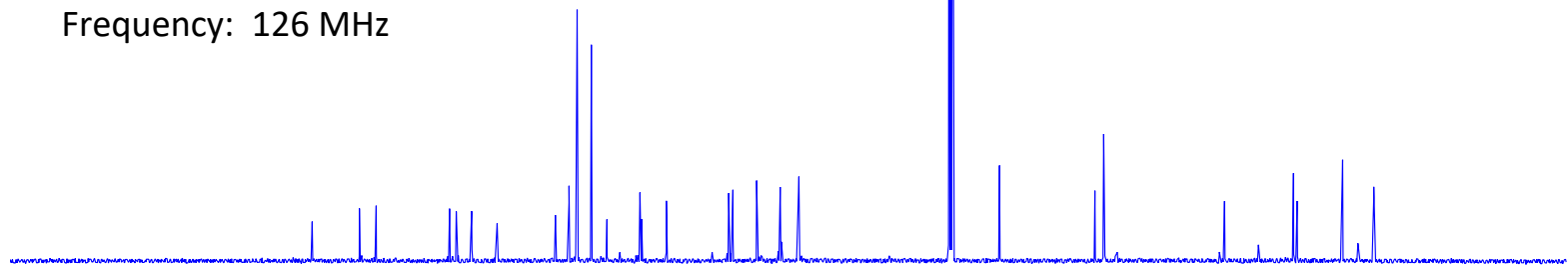

10

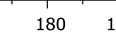

$160 \quad 150 \quad 140$

$\begin{array}{llllll}130 & 120 & 110 & 100 & 90 & 80\end{array}$

70




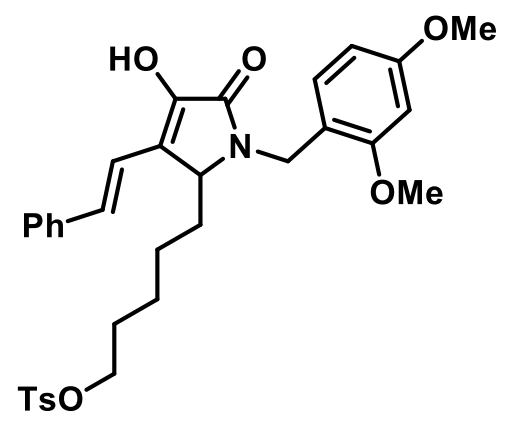

Yield: $23.7 \mathrm{mg}, 28 \%$.

Appearance: pale yellow oil.

$\boldsymbol{R}_{\mathbf{f}}=0.13$ (1:1 ethyl acetate:hexanes).

${ }^{1} \mathrm{H}$ NMR $\left(500 \mathrm{MHz}, \mathrm{CDCl}_{3}\right) \delta 7.78(\mathrm{br} \mathrm{s}, 1 \mathrm{H}), 7.74(\mathrm{~d}, J=8.3 \mathrm{~Hz}, 2 \mathrm{H}), 7.43(\mathrm{~d}, J=7.5 \mathrm{~Hz}, 2 \mathrm{H}), 7.35-7.29(\mathrm{~m}$, $4 \mathrm{H}), 7.24(\mathrm{dd}, J=6.9,1.8 \mathrm{~Hz}, 1 \mathrm{H}), 7.15(\mathrm{~d}, J=8.0 \mathrm{~Hz}, 1 \mathrm{H}), 6.90(\mathrm{~d}, J=16.5 \mathrm{~Hz}, 1 \mathrm{H}), 6.73(\mathrm{~d}, J=16.6 \mathrm{~Hz}, 1 \mathrm{H})$, 6.47-6.43 (m, 2H), $4.96(\mathrm{~d}, J=15.0 \mathrm{~Hz}, 1 \mathrm{H}), 4.22(\mathrm{~d}, J=15.0 \mathrm{~Hz}, 1 \mathrm{H}), 4.11(\mathrm{t}, J=6.4 \mathrm{~Hz}, 1 \mathrm{H}), 3.94(\mathrm{t}, J=4.2$ $\mathrm{Hz}, 2 \mathrm{H}), 3.83(\mathrm{~s}, 3 \mathrm{H}), 3.80(\mathrm{~s}, 3 \mathrm{H}), 2.42(\mathrm{~s}, 3 \mathrm{H}), 1.75-1.58(\mathrm{~m}, 2 \mathrm{H}), 1.57-1.49(\mathrm{~m}, 2 \mathrm{H}), 1.28-1.19(\mathrm{~m}, 2 \mathrm{H})$, $0.90-0.82(\mathrm{~m}, 2 \mathrm{H})$.

${ }^{13} \mathrm{C} \mathrm{NMR}\left(176 \mathrm{MHz}, \mathrm{CDCl}_{3}\right) \delta 167.1,160.6,158.3,144.6,143.3,137.1,133.1,130.9,130.6129 .8(2 \mathrm{C}), 128.7$ (2C), 127.8 (2C), 126.4 (2C), 120.9, 117.6, 117.2, 104.4, 98.4, 70.5, 56.5, 55.4, 55.4, 38.1, 28.8, 28.6, 25.4, 25.3, 21.6, 20.6.

IR (Diamond-ATR, neat) $\tilde{V}_{\max }: 2920,2850,1696,1677,1612,1590,1208,1174,1032,753$.

HRMS (ESI) m/z: [M-H]. Calcd for $\mathrm{C}_{33} \mathrm{H}_{36} \mathrm{NO}_{7} \mathrm{~S} 590.2218$; Found 590.2211. 


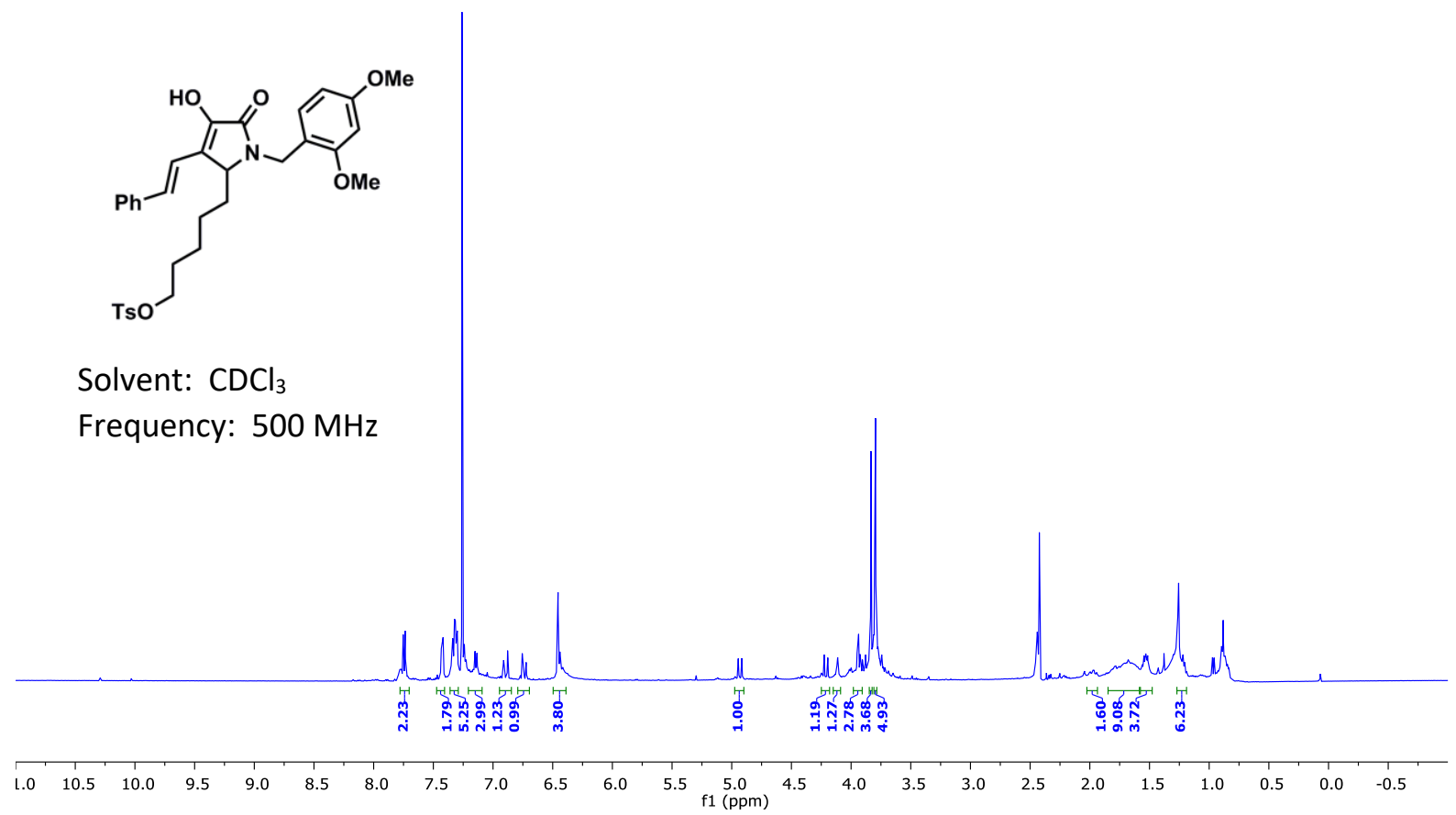

Feb02-2020-NM-20-067.18.fid
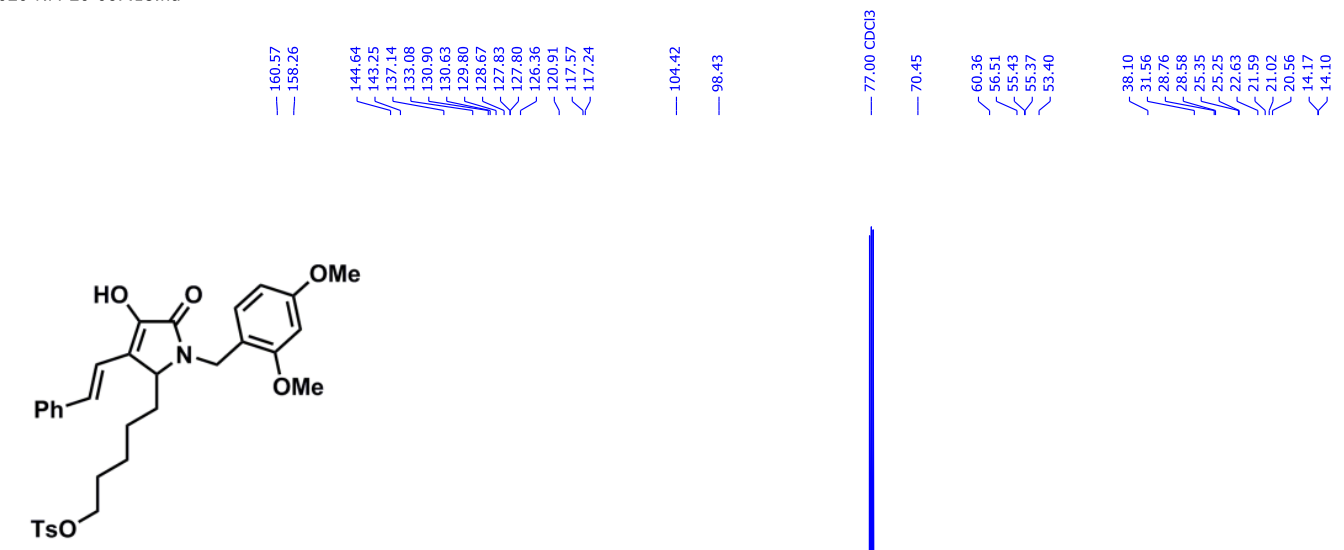

Solvent: $\mathrm{CDCl}_{3}$

Frequency: $176 \mathrm{MHz}$

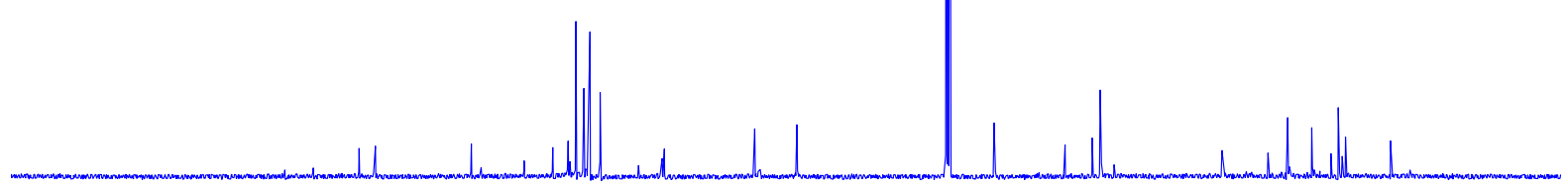

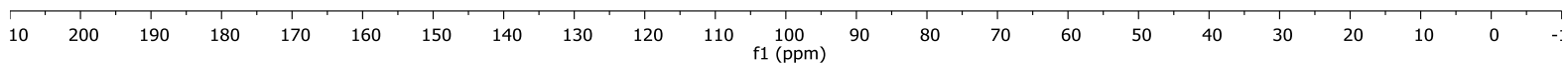




\section{General Procedure 3: Synthesis of bicyclic pyrrole-2,3-diones 5a-5n.}

To a solution of 4 (1.0 equiv) in dichloromethane $(0.04 \mathrm{M})$ was added anhydrous potassium carbonate (2.0 equiv). Reaction was stirred at reflux in a sand bath until full consumption of starting material was observed on (TLC) (2-24 h). The reaction was cooled to room temperature, diluted with dichloromethane and washed with saturated aqueous ammonium chloride solution and subsequently washed with saturated aqueous sodium bicarbonate solution. Organic layer was filtered over sodium sulfate and concentrated to volume. Often times, products did not require further purification, although purification was accomplished with flash column chromatography eluting with a gradient of hexanes to 1:1 ethyl acetate:hexanes providing product 5. (Note: products $5 \mathbf{e}, 5 \mathbf{5}, \mathbf{5 I}$ and $\mathbf{5 n}$ required 6 equiv $\mathrm{K}_{2} \mathrm{CO}_{3}$ )

(E)-1-(2,4-diMethoxybenzyl)-3a-styrylhexahydro-1H-indole-2,3-dione (5a).

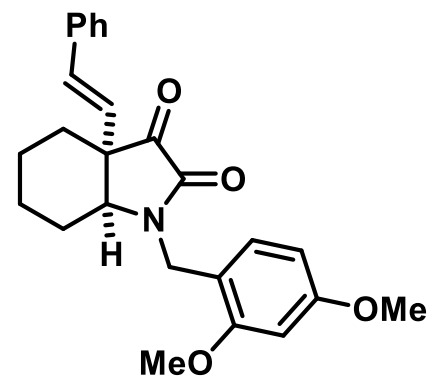

Yield: $9.7 \mathrm{mg}, 92 \%$.

Appearance: pale yellow oil.

$\boldsymbol{R}_{\mathbf{f}}=0.46$ (1:1 ethyl acetate:hexanes).

${ }^{1} \mathrm{H}$ NMR $\left(500 \mathrm{MHz}, \mathrm{CDCl}_{3}\right) \delta 7.27-7.25(\mathrm{~m}, 2 \mathrm{H}), 7.23-7.20(\mathrm{~m}, 2 \mathrm{H}), 7.14(\mathrm{dd}, J=8.2,1.5 \mathrm{~Hz}, 2 \mathrm{H}), 6.48-6.44$ $(\mathrm{m}, 2 \mathrm{H}), 6.18(\mathrm{~d}, J=16.3 \mathrm{~Hz}, 1 \mathrm{H}), 5.78(\mathrm{~d}, J=16.3 \mathrm{~Hz}, 1 \mathrm{H}), 5.13(\mathrm{~d}, J=14.3 \mathrm{~Hz}, 1 \mathrm{H}), 4.25(\mathrm{~d}, J=14.3 \mathrm{~Hz}, 1 \mathrm{H})$, $3.82(\mathrm{~s}, 3 \mathrm{H}), 3.73(\mathrm{~s}, 3 \mathrm{H}), 3.61(\mathrm{dd}, J=7.7,5.4 \mathrm{~Hz}, 1 \mathrm{H}), 2.33-2.27(\mathrm{~m}, 1 \mathrm{H}), 2.27-2.21(\mathrm{~m}, 1 \mathrm{H}), 1.70-1.52$ $(\mathrm{m}, 4 \mathrm{H}), 1.40-1.27(\mathrm{~m}, 2 \mathrm{H})$.

${ }^{13} \mathrm{C}$ NMR $\left(176 \mathrm{MHz}, \mathrm{CDCl}_{3}\right) \delta 199.3,161.1,159.7,158.8,136.0,132.0,131.8,128.5(2 \mathrm{C}), 128.4,128.1,126.3$ (2C), 115.2, 104.5, 98.6, 57.2, 55.4, 55.4, 52.0, 39.9, 29.6, 29.6, 21.5, 20.5.

IR (Diamond-ATR, neat) $\tilde{V}_{\text {max }}$ 2926, 2855, 1759, 1715, 1614, 1506, 1457, 1208, 1159, 1032.

HRMS (ESI) m/z: [M+H] $]^{+}$Calcd for $\mathrm{C}_{25} \mathrm{H}_{28} \mathrm{NO}_{4}$ 406.2013; Found 406.2016. 
Feb12-2020-NM-20-047pure.11.fic
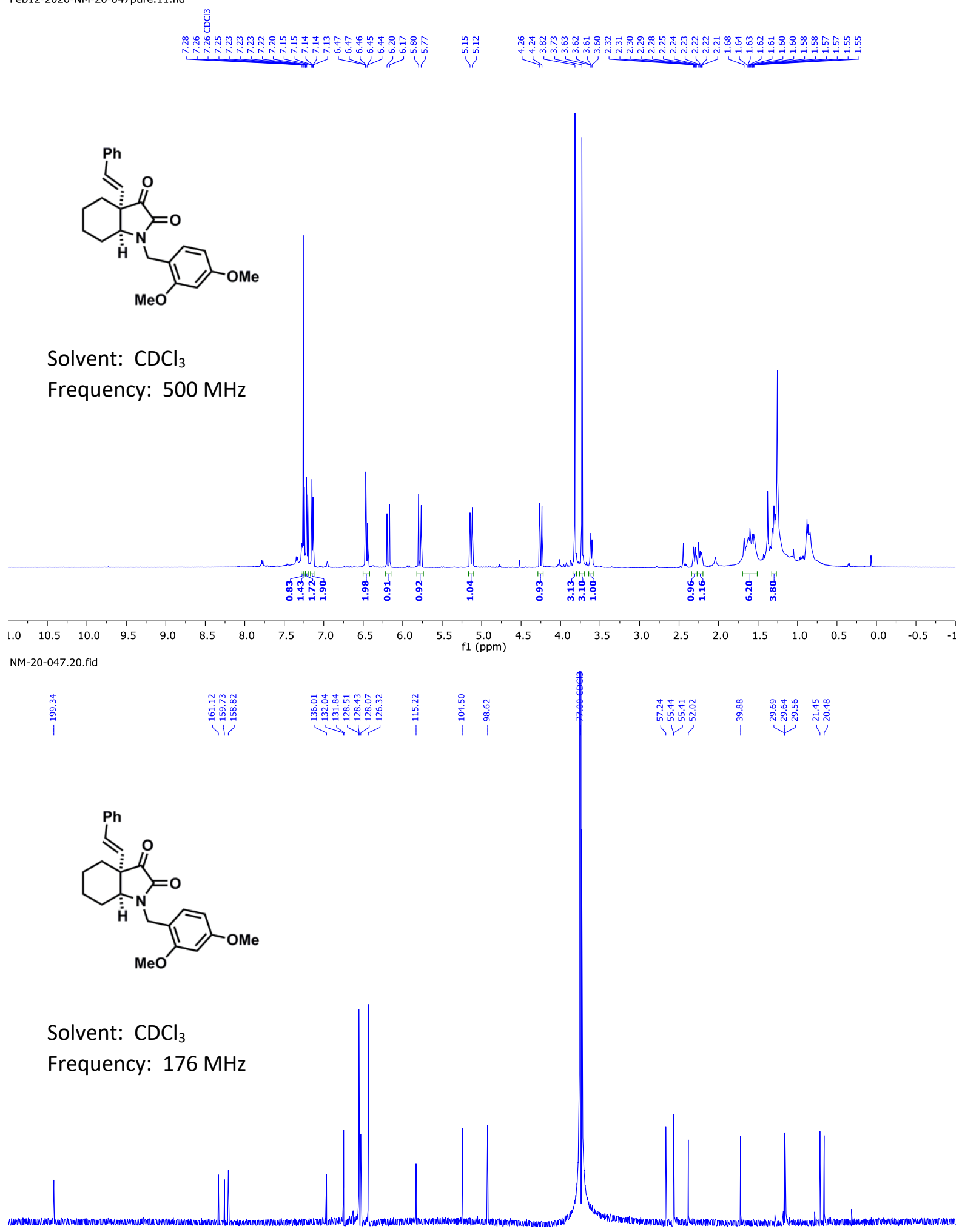

$\begin{array}{lllllllllllllllllllllll}10 & 200 & 190 & 180 & 170 & 160 & 150 & 140 & 130 & 120 & 110 & 100 & 90 & 80 & 70 & 60 & 50 & 40 & 30 & 20 & 10 & 0 & -\end{array}$ 

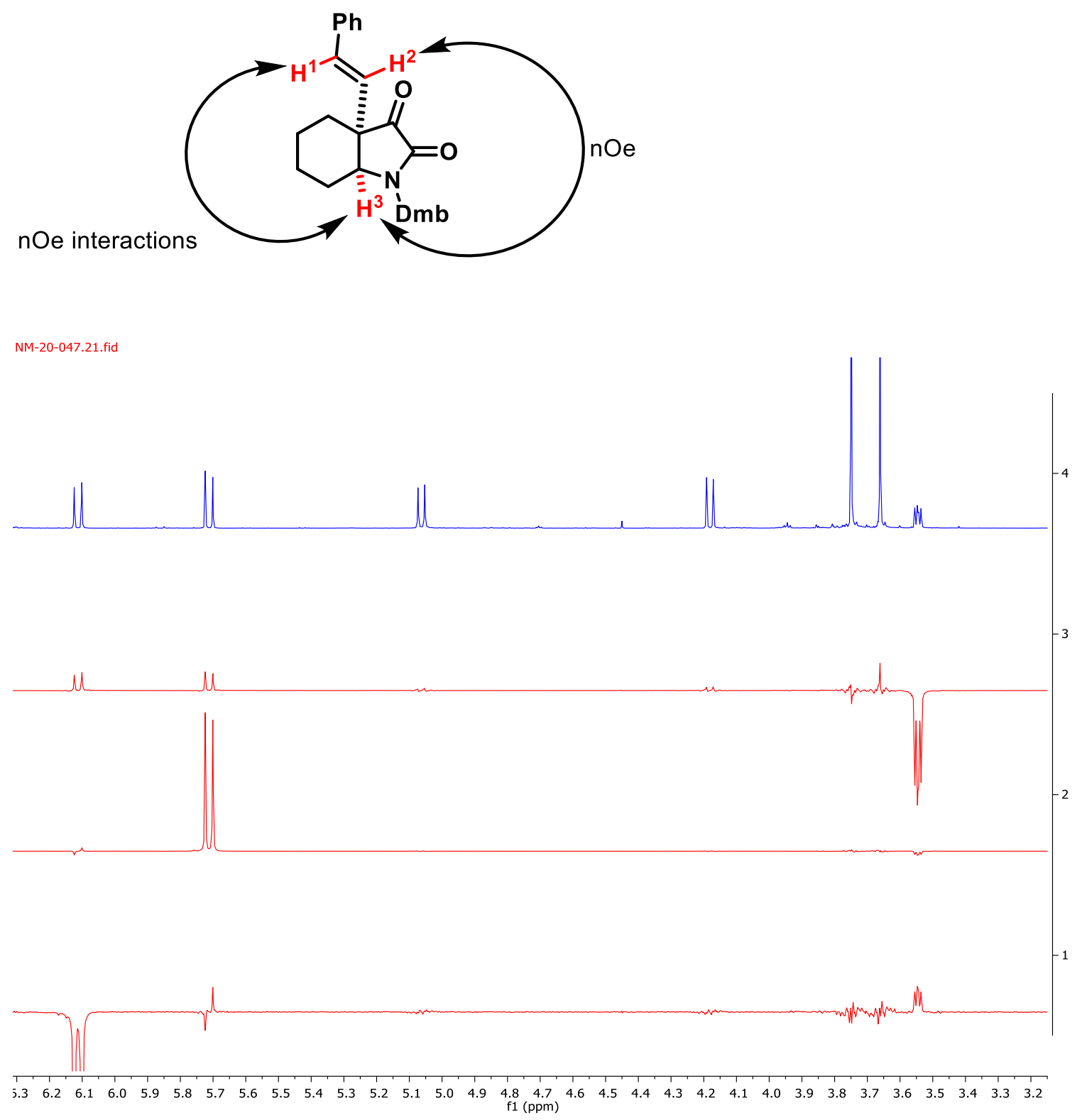
(E)-1-(2-Nitrobenzyl)-3a-styrylhexahydro-1H-indole-2,3-dione. (5b)

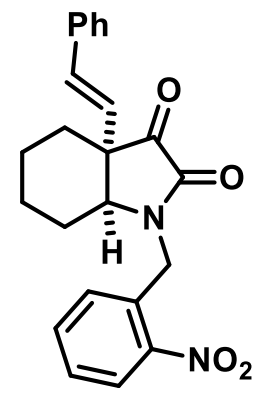

Yield: $7.0 \mathrm{mg}, 69 \%$.

Appearance: white solid.

$\boldsymbol{R}_{\mathrm{f}}=0.66$ (1:1 ethyl acetate:hexanes).

${ }^{1} \mathbf{H}$ NMR $\left(500 \mathrm{MHz}, \mathrm{CDCl}_{3}\right) \delta 8.06-8.01(\mathrm{~m}, 1 \mathrm{H}), 7.52-7.43(\mathrm{~m}, 3 \mathrm{H}), 7.33-7.27(\mathrm{~m}, 3 \mathrm{H}), 7.21(\mathrm{~d}, J=7.1 \mathrm{~Hz}$, $2 \mathrm{H}), 6.30(\mathrm{~d}, J=16.3 \mathrm{~Hz}, 1 \mathrm{H}), 5.92(\mathrm{~d}, J=16.3 \mathrm{~Hz}, 1 \mathrm{H}), 5.28(\mathrm{~d}, J=7.1 \mathrm{~Hz}, 1 \mathrm{H}), 4.82(\mathrm{~d}, J=15.9 \mathrm{~Hz}, 1 \mathrm{H}), 3.80$ (dd, $J=7.7,5.2 \mathrm{~Hz}, 1 \mathrm{H}), 2.36-2.28(\mathrm{~m}, 2 \mathrm{H}), 1.71-1.50(\mathrm{~m}, 3 \mathrm{H}), 1.39-1.21(\mathrm{~m}, 3 \mathrm{H})$.

${ }^{13} \mathrm{C}$ NMR $\left(176 \mathrm{MHz}, \mathrm{CDCl}_{3}\right) \delta 198.4,160.9,148.7,135.6,133.8,133.0,130.8,130.7,129.1,128.7(2 \mathrm{C})$, $128.4,128.4,126.3(2 \mathrm{C}), 125.1,59.3,52.7,42.0,30.3,29.9,21.5,20.7$.

IR (Diamond-ATR, neat) $\tilde{V}_{\text {max }}: 2933,2855,1763,1715,1524,1442,1346,1059,745$.

HRMS (ESI) m/z: [M+H] ${ }^{+}$Calcd for $\mathrm{C}_{23} \mathrm{H}_{23} \mathrm{~N}_{2} \mathrm{O}_{4}$ 391.1652; Found 391.1643. 
<smiles>O=C1C(=O)N(Cc2ccccc2[N+](=O)[O-])C2(/C=C/c3ccccc3)CCCCC12</smiles>

Solvent: $\mathrm{CDCl}_{3}$

Frequency: $500 \mathrm{MHz}$

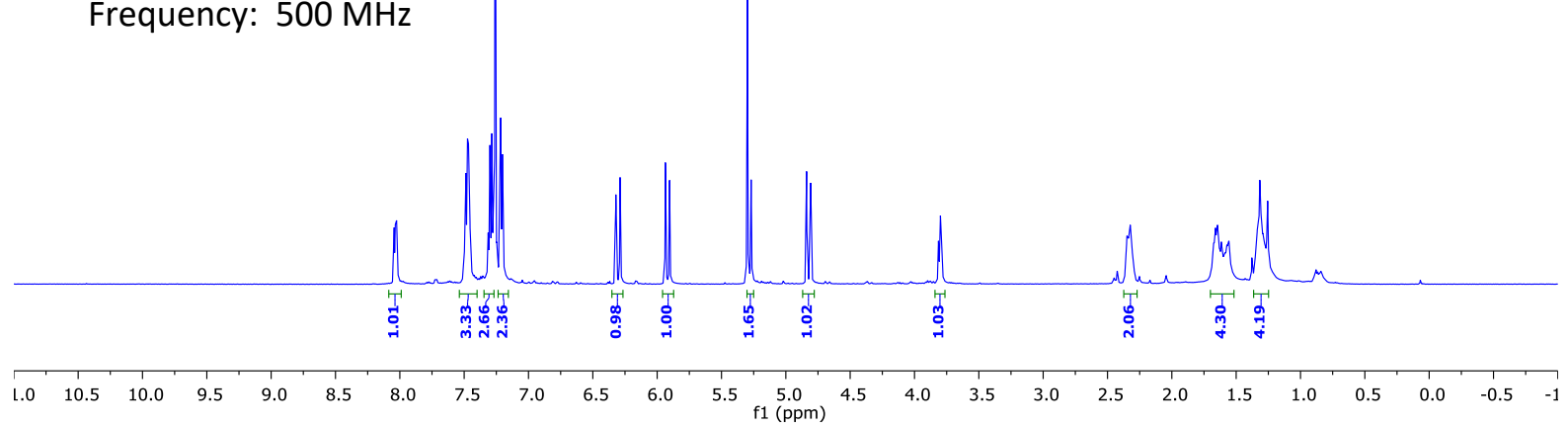

NM-20-050Rf0.6.11.fid
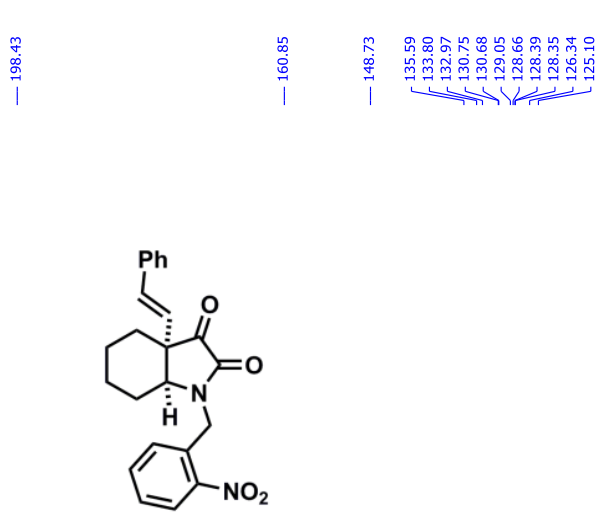

Solvent: $\mathrm{CDCl}_{3}$

Frequency: $176 \mathrm{MHz}$

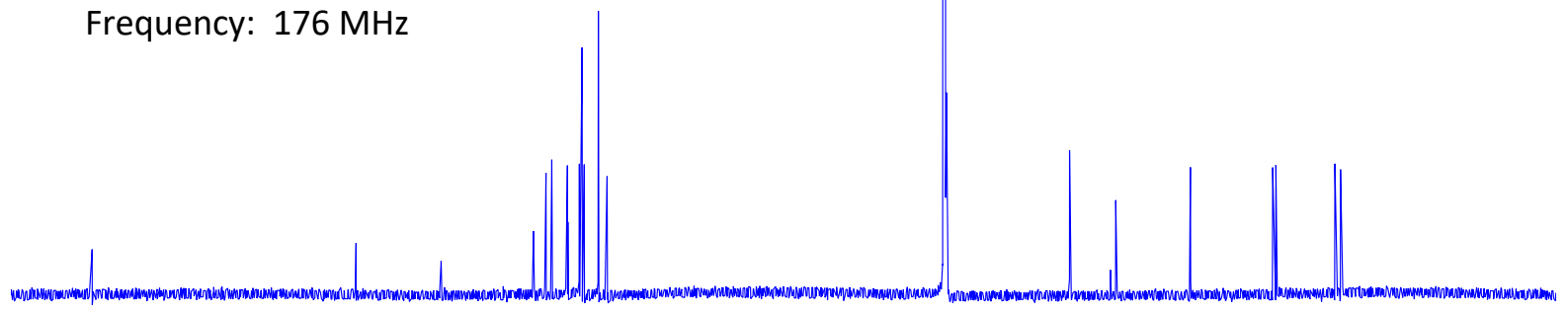

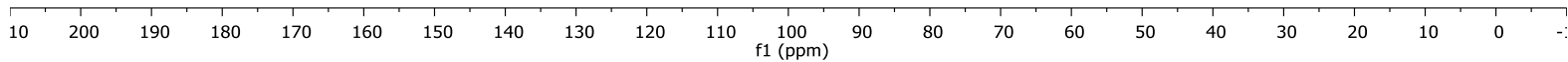


(E)-1-Benzyl-3a-styrylhexahydro-1H-indole-2,3-dione (5c).<smiles>O=C1C(=O)[C@]2(/C=C/c3ccccc3)CCCC[C@H]2N1Cc1ccccc1</smiles>

Yield: $9.0 \mathrm{mg}$, quantitative.

Appearance: off-white waxy solid.

$\boldsymbol{R}_{\mathrm{f}}=0.67$ (1:1 ethyl acetate:hexanes).

${ }^{1} \mathrm{H}$ NMR $\left(500 \mathrm{MHz}, \mathrm{CDCl}_{3}\right) \delta$ 7.38-7.34 (m, 3H), 7.33-7.29 (m, 2H), 7.29-7.27 (m, 1H), 7.25-7.23 (m, 1H), $7.18(\mathrm{dd}, J=6.8,1.8 \mathrm{~Hz}, 2 \mathrm{H}), 6.26(\mathrm{~d}, J=16.3 \mathrm{~Hz}, 1 \mathrm{H}), 5.84(\mathrm{~d}, J=16.3 \mathrm{~Hz}, 1 \mathrm{H}), 5.28(\mathrm{~d}, J=14.6 \mathrm{~Hz}, 2 \mathrm{H})$, $4.15(\mathrm{~d}, J=14.6 \mathrm{~Hz}, 1 \mathrm{H}), 3.65(\mathrm{dd}, J=7.4,5.4 \mathrm{~Hz}, 1 \mathrm{H}), 2.32-2.23(\mathrm{~m}, 1 \mathrm{H}), 2.22-2.14(\mathrm{~m}, 1 \mathrm{H}), 1.68-1.56(\mathrm{~m}$, $2 \mathrm{H}), 1.55-1.39(\mathrm{~m}, 2 \mathrm{H}), 1.38-1.29(\mathrm{~m}, 2 \mathrm{H})$.

${ }^{13} \mathrm{C}$ NMR $\left(176 \mathrm{MHz}, \mathrm{CDCl}_{3}\right) \delta 198.9,160.0,135.8,134.9,132.6,129.0(2 \mathrm{C}), 128.7(2 \mathrm{C}), 128.6(2 \mathrm{C}), 128.3$, $128.2,127.9,126.3$ (2C), 57.2, 52.0, 45.5, 29.7, 29.1, 21.3, 20.3.

IR (Diamond-ATR, neat) $\tilde{V}_{\max }:$ 2930, 2855, 1759, 1711, 1446, 965, 745, 701.

HRMS (ESI) m/z: [M+H] ${ }^{+}$Calcd for $\mathrm{C}_{23} \mathrm{H}_{24} \mathrm{NO}_{2}$ 346.1802; Found 346.1806. 
<smiles>O=C1C(=O)[C@]2(/C=C/c3ccccc3)CCCC[C@H]2N1Cc1ccccc1</smiles>
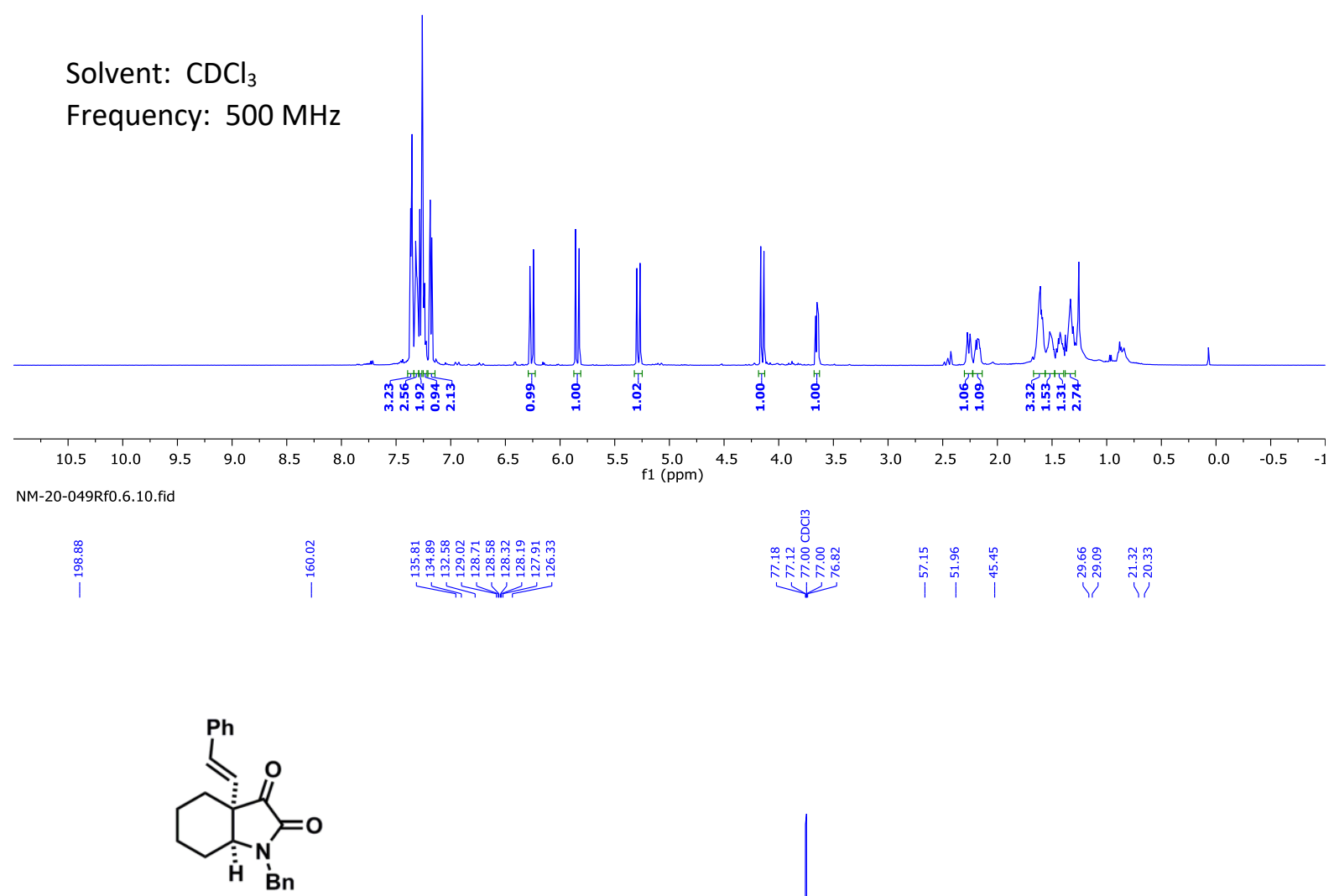

Solvent: $\mathrm{CDCl}_{3}$

Frequency: $176 \mathrm{MHz}$
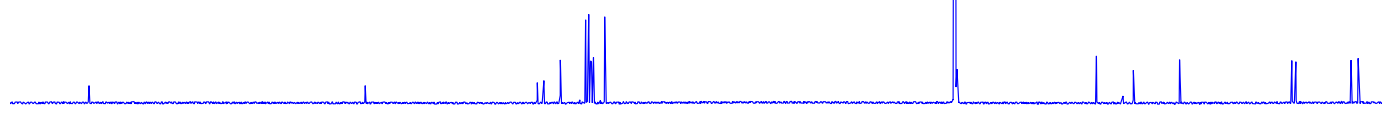
(E)-1-(4-Bromobenzyl)-3a-styrylhexahydro-1H-indole-2,3-dione (5d).

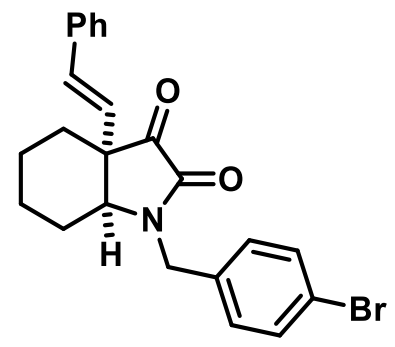

Yield: $8.8 \mathrm{mg}, 80 \%$.

Appearance: pale yellow viscous oil.

$\boldsymbol{R}_{\mathrm{f}}=0.63$ (1:1 ethyl acetate:hexanes).

${ }^{1} \mathrm{H}$ NMR $\left(500 \mathrm{MHz}, \mathrm{CDCl}_{3}\right) \delta 7.47(\mathrm{~d}, J=8.3 \mathrm{~Hz}, 2 \mathrm{H}), 7.30(\mathrm{t}, J=7.1 \mathrm{~Hz}, 2 \mathrm{H}), 7.27-7.26(\mathrm{~m}, 1 \mathrm{H}), 7.19(\mathrm{~d}, J=$ $8.6 \mathrm{~Hz}, 2 \mathrm{H}), 7.17(\mathrm{~d}, J=7.2 \mathrm{~Hz}, 2 \mathrm{H}), 6.24(\mathrm{~d}, J=16.3 \mathrm{~Hz}, 1 \mathrm{H}), 5.82(\mathrm{~d}, J=16.4 \mathrm{~Hz}, 1 \mathrm{H}), 5.19(\mathrm{~d}, J=14.7 \mathrm{~Hz}$, $1 \mathrm{H}), 4.13(\mathrm{~d}, J=14.7 \mathrm{~Hz}, 1 \mathrm{H}), 3.64(\mathrm{dd}, J=7.3,5.3 \mathrm{~Hz}, 1 \mathrm{H}), 2.29-2.22(\mathrm{~m}, 1 \mathrm{H}), 2.21-2.14(\mathrm{~m}, 1 \mathrm{H}), 1.67-$ $1.45(\mathrm{~m}, 3 \mathrm{H}), 1.44-1.29(\mathrm{~m}, 3 \mathrm{H})$.

${ }^{13} \mathrm{C}$ NMR $\left(176 \mathrm{MHz}, \mathrm{CDCl}_{3}\right) \delta$ 198.6, 160.1, 135.6, 134.1, 132.7, 132.2 (2C), $130.4(2 \mathrm{C}), 128.7$ (2C), 128.3, $127.7,126.3(2 \mathrm{C}), 122.4,57.4,52.1,44.9,29.7,29.2,21.3,20.4$.

IR (Diamond-ATR, neat) $\tilde{V}_{\max }:$ 2933, 2859, 1759, 1711, 1487, 1442, 1405, 1070, 965, 742.

HRMS (ESI) m/z: [M+H] $]^{+}$Calcd for $\mathrm{C}_{23} \mathrm{H}_{23} \mathrm{BrNO}_{2}$ 424.0907; Found 424.0916. 
Feb12-2020-NM-20-051pure.13.fid
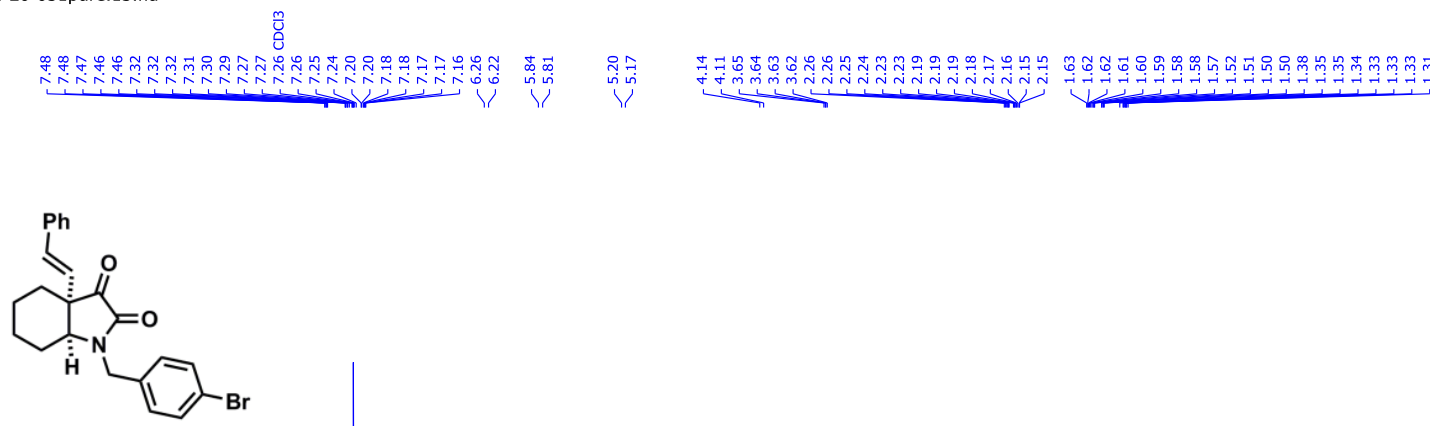

Solvent: $\mathrm{CDCl}_{3}$

Frequency: $500 \mathrm{MHz}$

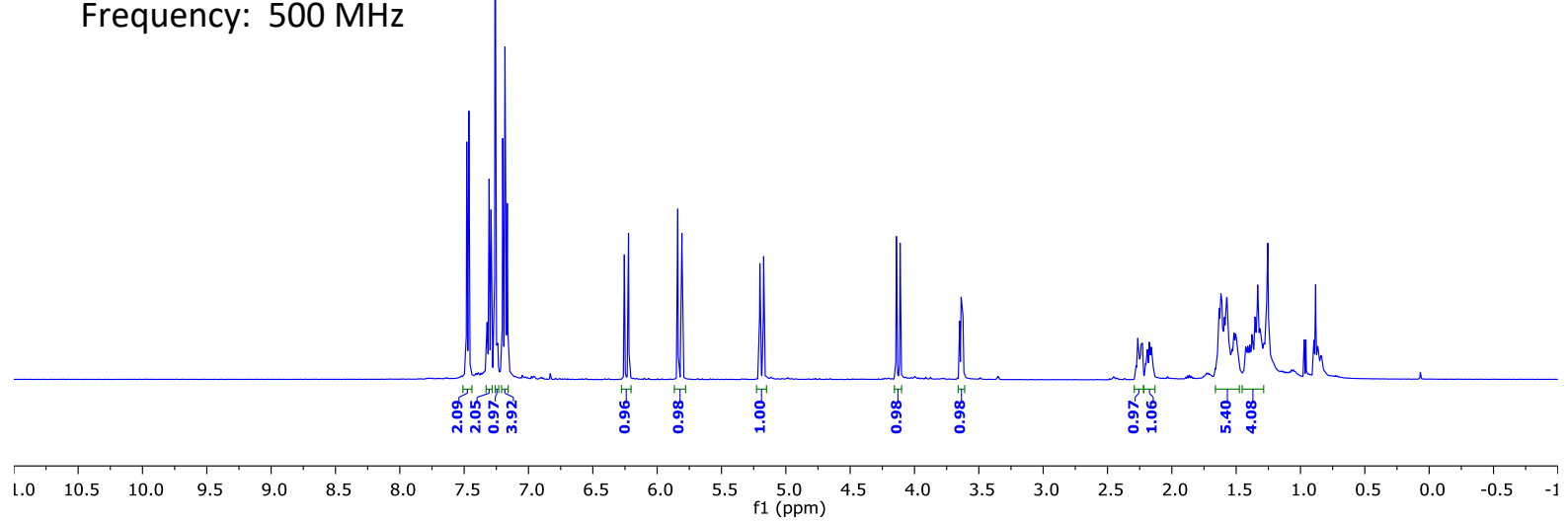

NM-20-051Rf0.6.12.fid
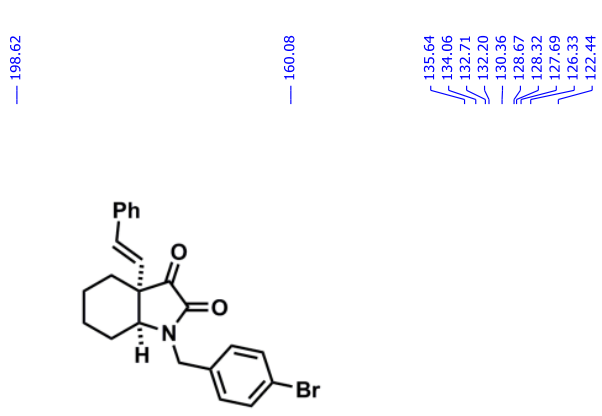

Solvent: $\mathrm{CDCl}_{3}$

Frequency: $176 \mathrm{MHz}$

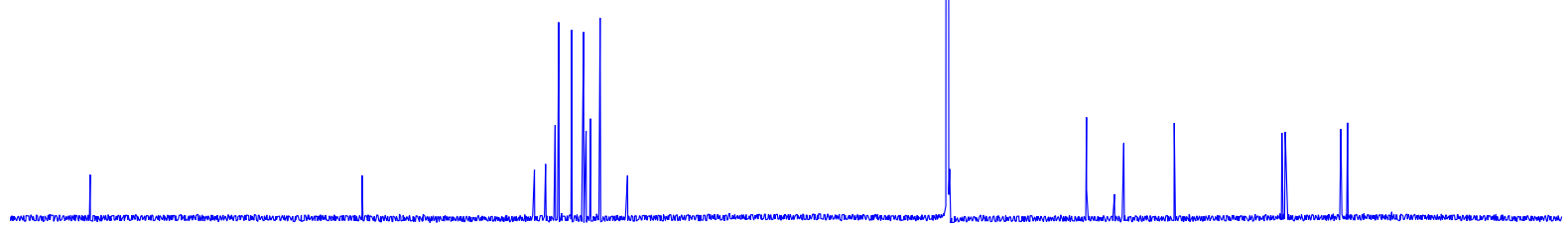

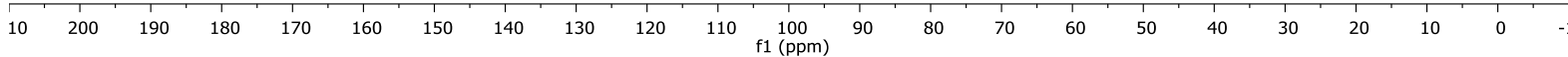


(E)-1-(Benzo[d][1,3]dioxol-5-ylmethyl)-3a-styrylhexahydro-1H-indole-2,3-dione (5e).<smiles>O=C1C(=O)[C@]2(/C=C/c3ccccc3)CCCC[C@H]2N1Cc1ccc2c(c1)OCO2</smiles>

Yield: $5.7 \mathrm{mg}, 56 \%$.

Appearance: white solid.

$\boldsymbol{R}_{\mathrm{f}}=0.48$ (1:1 ethyl acetate:hexanes).

${ }^{1} \mathrm{H}$ NMR $\left(500 \mathrm{MHz}, \mathrm{CDCl}_{3}\right) \delta$ 7.30-7.26 (m, 2H), 7.25-7.22 (m, 1H), 7.22-7.19 (m, 2H), 6.82-6.75 (m, 3H), $6.27(\mathrm{~d}, J=16.3 \mathrm{~Hz}, 1 \mathrm{H}), 5.96(\mathrm{dd}, J=23.1,1.5 \mathrm{~Hz}, 2 \mathrm{H}), 5.86(\mathrm{~d}, J=16.3 \mathrm{~Hz}, 1 \mathrm{H}), 5.18(\mathrm{~d}, J=14.5 \mathrm{~Hz}, 1 \mathrm{H})$, $4.06(\mathrm{~d}, J=14.5 \mathrm{~Hz}, 1 \mathrm{H}), 3.67$ (dd, $J=7.5,5.4 \mathrm{~Hz}, 1 \mathrm{H}), 2.29-2.24(\mathrm{~m}, 1 \mathrm{H}), 2.18$ (dddd, $J=14.8,7.6,5.3,3.0$ $\mathrm{Hz}, 1 \mathrm{H}), 1.67-1.56(\mathrm{~m}, 2 \mathrm{H}), 1.56-1.49(\mathrm{~m}, 1 \mathrm{H}), 1.46-1.39(\mathrm{~m}, 1 \mathrm{H}), 1.37-1.30(\mathrm{~m}, 2 \mathrm{H})$.

${ }^{13} \mathrm{C} \mathrm{NMR}\left(176 \mathrm{MHz}, \mathrm{CDCl}_{3}\right) \delta 198.9,159.9,148.3,147.7,135.8,132.6,128.6$ (2C), 128.6, 128.2, 127.9, 126.4 (2C), 122.2, 109.1, 108.5, 101.3, 57.0, 52.0, 45.3, 29.6, 29.2, 21.4, 20.4.

IR (Diamond-ATR, neat) $\tilde{V}_{\max }:$ 2930, 2870, 1759, 1707, 1495, 1446, 1245, 1036, 928, 745.

HRMS (ESI) m/z: [M+H] ${ }^{+}$Calcd for $\mathrm{C}_{24} \mathrm{H}_{24} \mathrm{NO}_{4}$ 390.1700; Found 390.1702. 


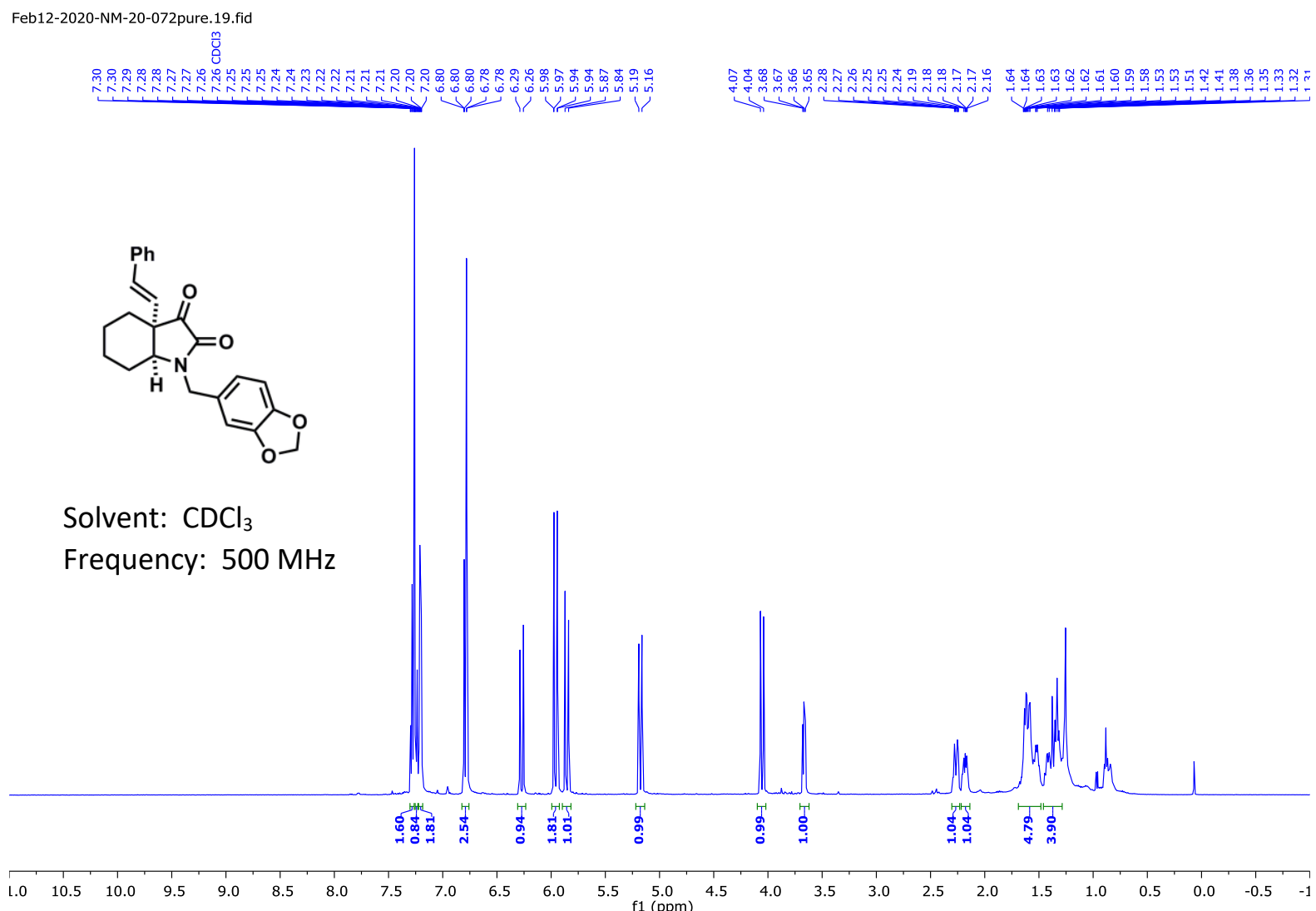

NM-20-072.14.fid
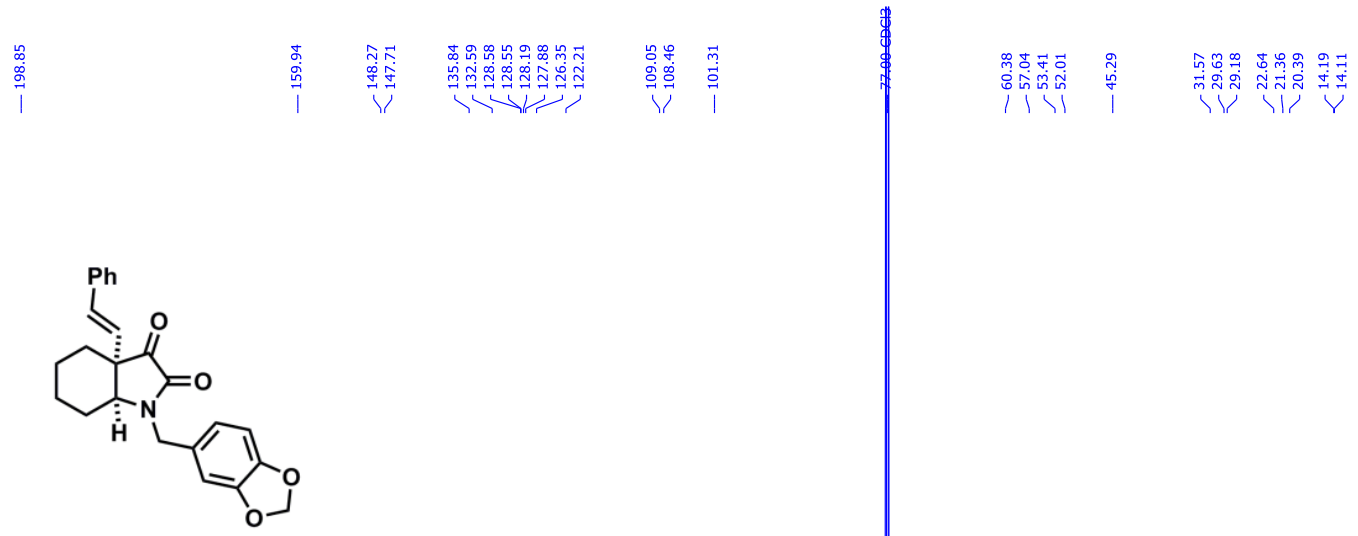

Solvent: $\mathrm{CDCl}_{3}$

Frequency: $176 \mathrm{MHz}$

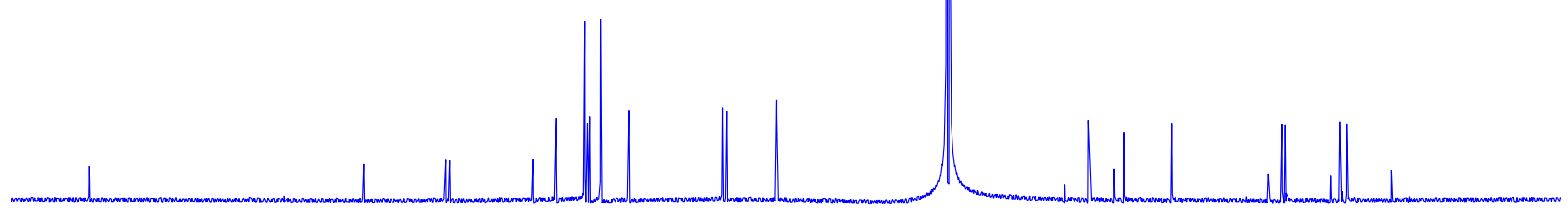

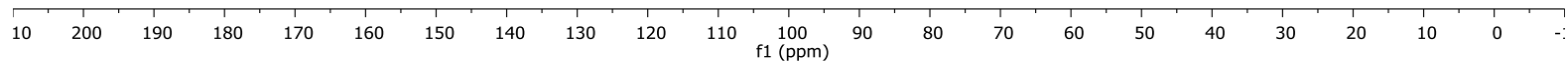


(E)-3a-Styryl-1-(thiophen-2-ylmethyl)hexahydro-1H-indole-2,3-dione (5f).

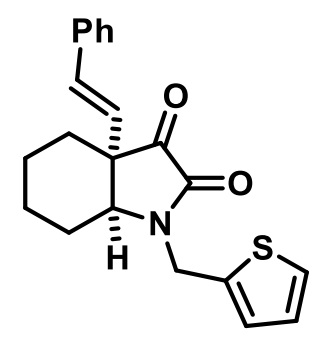

Yield: $9.1 \mathrm{mg}$, quantitative.

Appearance: pale yellow oil.

$\boldsymbol{R}_{\mathrm{f}}=0.61$ (1:1 ethyl acetate:hexanes).

${ }^{1} \mathbf{H}$ NMR $\left(500 \mathrm{MHz}, \mathrm{CDCl}_{3}\right) \delta 7.32(\mathrm{dd}, J=5.1,0.9 \mathrm{~Hz}, 1 \mathrm{H}), 7.30-7.26(\mathrm{~m}, 2 \mathrm{H}), 7.25-7.19(\mathrm{~m}, 3 \mathrm{H}), 7.09(\mathrm{~d}, J$ $=3.4 \mathrm{~Hz}, 1 \mathrm{H}), 7.02(\mathrm{dd}, J=5.1,3.5 \mathrm{~Hz}, 1 \mathrm{H}), 6.30(\mathrm{~d}, J=16.3 \mathrm{~Hz}, 1 \mathrm{H}), 5.90(\mathrm{~d}, J=16.3 \mathrm{~Hz}, 1 \mathrm{H}), 5.38(\mathrm{~d}, J=$ $15.2 \mathrm{~Hz}, 1 \mathrm{H}), 4.42(\mathrm{~d}, J=15.3 \mathrm{~Hz}, 1 \mathrm{H}), 3.78(\mathrm{dd}, J=7.2,5.3 \mathrm{~Hz}, 1 \mathrm{H}), 2.32-2.16(\mathrm{~m}, 2 \mathrm{H}), 1.64(\mathrm{ddt}, J=12.3$, $8.5,5.2 \mathrm{~Hz}, 2 \mathrm{H}), 1.60-1.43(\mathrm{~m}, 2 \mathrm{H}), 1.37(\mathrm{ddt}, J=11.6,5.4,3.0 \mathrm{~Hz}, 2 \mathrm{H})$.

${ }^{13} \mathrm{C} \mathrm{NMR}\left(176 \mathrm{MHz}, \mathrm{CDCl}_{3}\right) \delta 198.5,159.6,136.5,135.9,132.7,128.6$ (2C), 128.2, 128.1, 127.7, 127.2, 126.4 (2C), 126.4, 57.1, 51.9, 39.8, 29.6, 29.0, 21.3, 20.3.

IR (Diamond-ATR, neat) $\tilde{v}_{\max }:$ 2926, 2859, 1759, 1711, 1513, 1446, 1431, 1178, 1044, 965, 745, 697.

HRMS (ESI) m/z: [M+H] $]^{+}$Calcd for $\mathrm{C}_{21} \mathrm{H}_{22} \mathrm{NO}_{2} \mathrm{~S}$ 352.1366; Found 352.1371. 
Feb12-2020-NM-20-054pure.16.fid

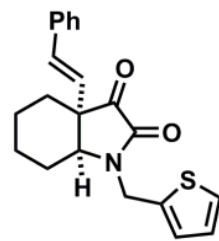

Solvent: $\mathrm{CDCl}_{3}$

Frequency: $500 \mathrm{MHz}$

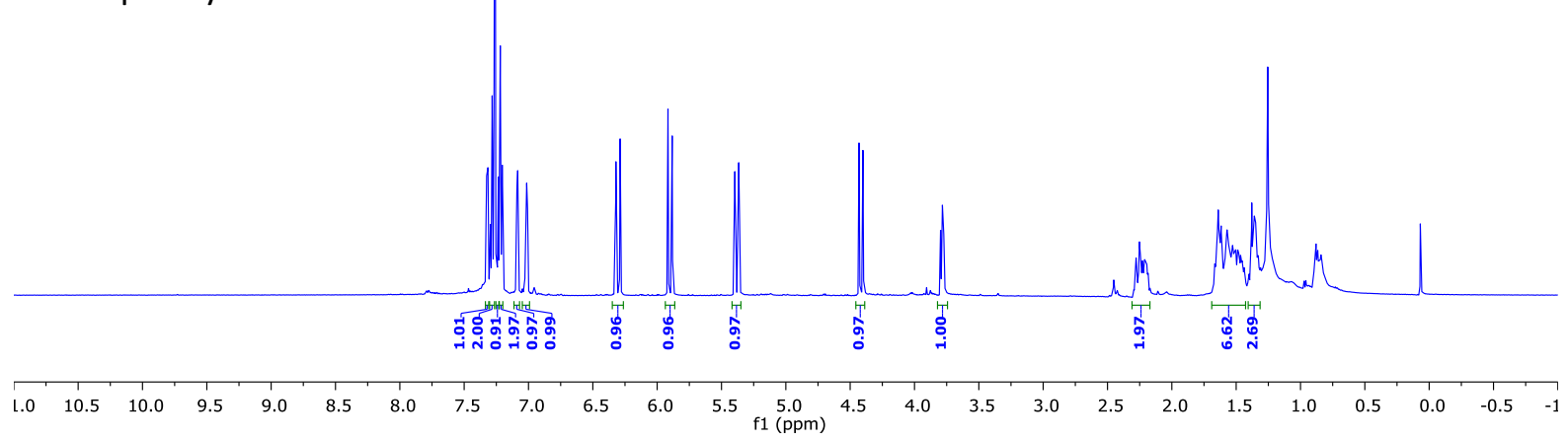

NM-20-054Rf0.6.15.fid
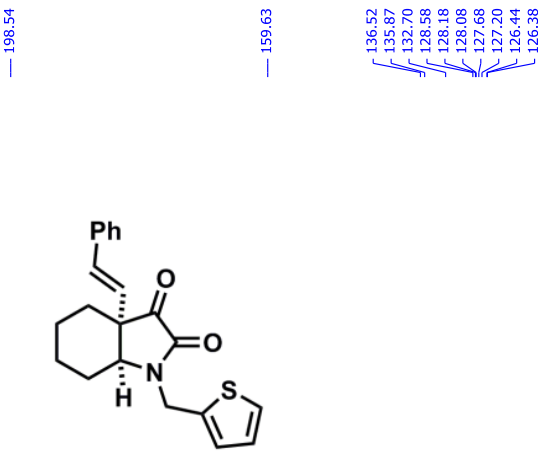

Solvent: $\mathrm{CDCl}_{3}$

Frequency: $176 \mathrm{MHz}$

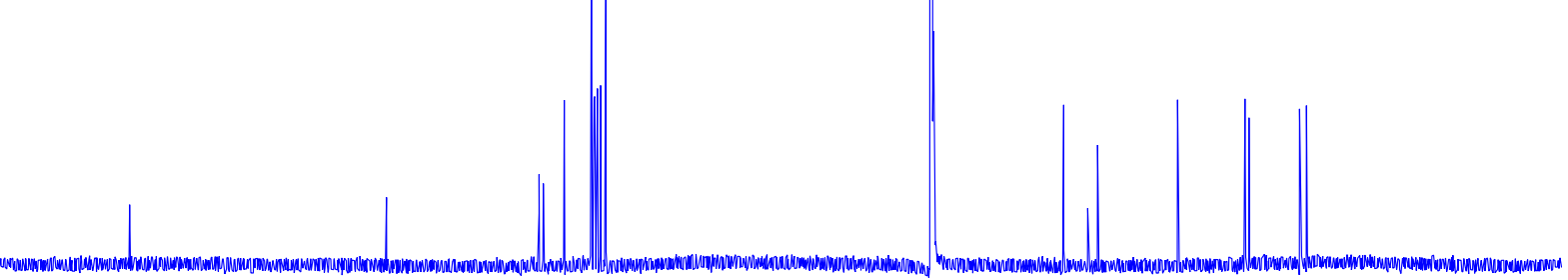

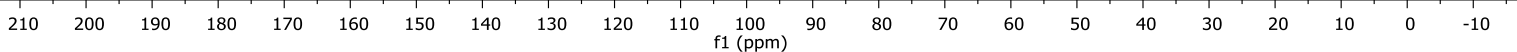


(E)-1-Allyl-3a-styrylhexahydro-1H-indole-2,3-dione (5g).

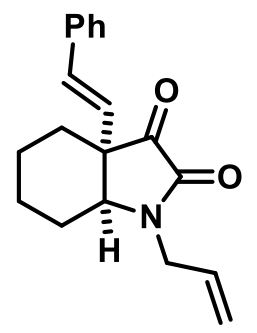

Yield: $7.7 \mathrm{mg}$, quantitative.

Appearance: pale yellow oil.

$\boldsymbol{R}_{\mathbf{f}}=0.46$ (1:1 ethyl acetate:hexanes).

${ }^{1}$ H NMR $\left(500 \mathrm{MHz}, \mathrm{CDCl}_{3}\right) \delta 7.33-7.27(\mathrm{~m}, 4 \mathrm{H}), 7.28-7.23(\mathrm{~m}, 1 \mathrm{H}), 6.47(\mathrm{~d}, J=16.3 \mathrm{~Hz}, 1 \mathrm{H}), 6.06(\mathrm{~d}, J=$ $16.3 \mathrm{~Hz}, 1 \mathrm{H}$ ), 5.82 (dddd, $J=17.5,10.1,7.8,5.1 \mathrm{~Hz}, 1 \mathrm{H}), 5.38-5.32(\mathrm{~m}, 2 \mathrm{H}), 4.59$ (ddt, $J=15.1,5.1,1.6 \mathrm{~Hz}$, $1 \mathrm{H}), 3.88(\mathrm{t}, J=5.9 \mathrm{~Hz}, 1 \mathrm{H}), 3.74(\mathrm{dd}, J=15.0,7.8 \mathrm{~Hz}, 1 \mathrm{H}), 2.25-2.12(\mathrm{~m}, 2 \mathrm{H}), 1.72-1.34(\mathrm{~m}, 6 \mathrm{H})$.

${ }^{13} \mathrm{C}$ NMR $\left(176 \mathrm{MHz}, \mathrm{CDCl}_{3}\right) \delta 199.0,159.9,135.9,132.7,131.0,128.7$ (2C), 128.2, 128.0, 126.4 (2C), 120.1, $57.6,51.6,44.4,29.8,28.3,21.1,20.0$.

IR (Diamond-ATR, neat) $\tilde{\mathbf{v}}_{\text {max }}:$ 2930, 2863, 1759, 1711, 1446, 1416, 1174, 965, 932, 745, 693.

HRMS (ESI) m/z: [M+H] $]^{+}$Calcd for $\mathrm{C}_{19} \mathrm{H}_{22} \mathrm{NO}_{2}$ 296.1645; Found 296.1652. 
<smiles>C=CCN1C(=O)C(=O)C2(/C=C/c3ccccc3)CCCC[C@@H]12</smiles>

Solvent: $\mathrm{CDCl}_{3}$

Frequency: $500 \mathrm{MHz}$

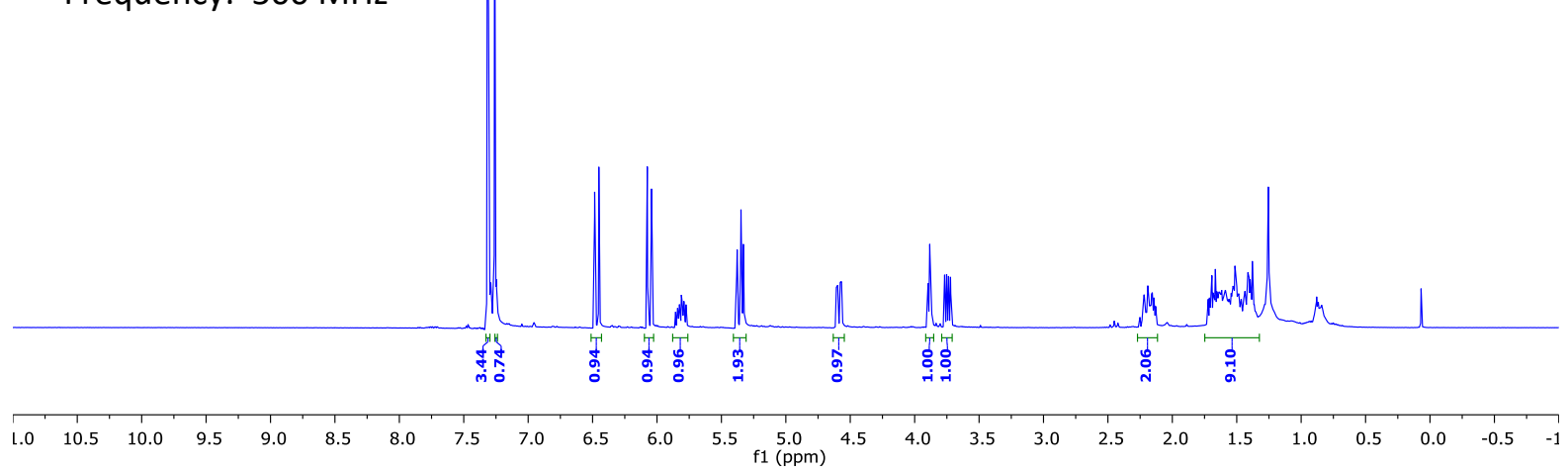

NM-20-052Rf0.6.13.fid
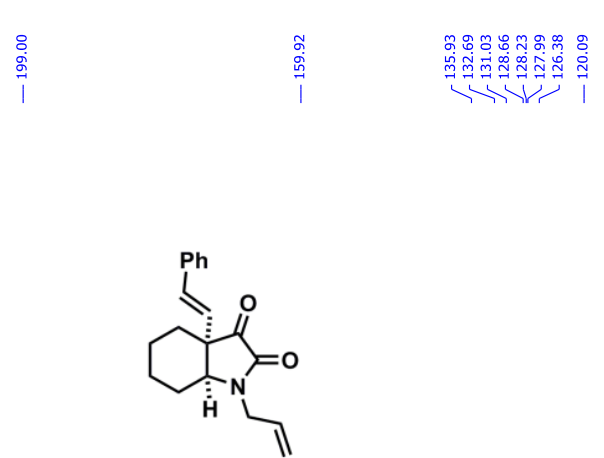

Solvent: $\mathrm{CDCl}_{3}$

Frequency: $176 \mathrm{MHz}$

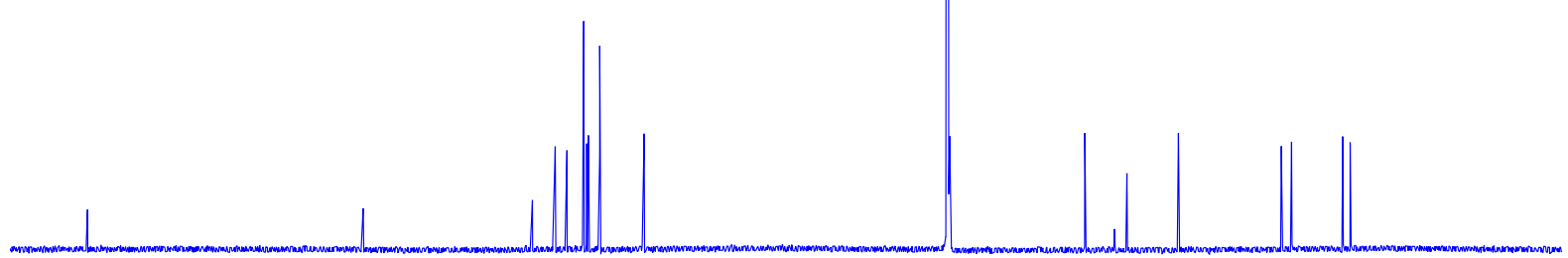

\begin{tabular}{llllllllllllllllllllllll}
\hline 10 & 200 & 190 & 180 & 170 & 160 & 150 & 140 & 130 & 120 & 110 & $\begin{array}{l}100 \\
\mathrm{f} 1(\mathrm{ppm})\end{array}$ & 90 & 80 & 70 & 60 & 50 & 40 & 30 & 20 & 10 & 0 & -
\end{tabular} 
(E)-1-(Prop-2-yn-1-yl)-3a-styrylhexahydro-1H-indole-2,3-dione (5h).

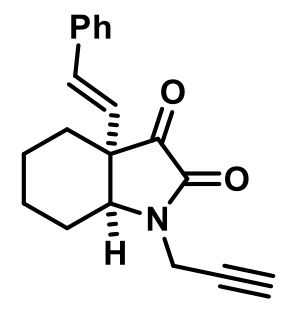

Yield: $6.9 \mathrm{mg}, 91 \%$.

Appearance: pale yellow oil.

$\boldsymbol{R}_{\mathbf{f}}=0.61$ (1:1 ethyl acetate:hexanes).

${ }^{1} \mathbf{H}$ NMR $\left(500 \mathrm{MHz}, \mathrm{CDCl}_{3}\right) \delta 7.36-7.30(\mathrm{~m}, 4 \mathrm{H}), 7.28-7.24(\mathrm{~m}, 1 \mathrm{H}), 6.57(\mathrm{~d}, J=16.3 \mathrm{~Hz}, 1 \mathrm{H}), 6.08(\mathrm{~d}, J=$ $16.3 \mathrm{~Hz}, 1 \mathrm{H}), 4.80(\mathrm{dd}, J=17.7,2.6 \mathrm{~Hz}, 1 \mathrm{H}), 4.13(\mathrm{dd}, J=6.6,5.1 \mathrm{~Hz}, 1 \mathrm{H}), 3.97(\mathrm{dd}, J=17.7,2.6 \mathrm{~Hz}, 1 \mathrm{H})$, $2.37(\mathrm{t}, J=2.6 \mathrm{~Hz}, 1 \mathrm{H}$ ), 2.21 (dddd, $J=18.0,13.7,7.2,3.8 \mathrm{~Hz}, 2 \mathrm{H}$ ), 1.72 (ddd, $J=13.8,9.0,4.5 \mathrm{~Hz}, 1 \mathrm{H}$ ), $1.68-$ $1.45(\mathrm{~m}, 4 \mathrm{H}), 1.45-1.36(\mathrm{~m}, 1 \mathrm{H})$.

${ }^{13} \mathrm{C}$ NMR (176 MHz, $\left.\mathrm{CDCl}_{3}\right) \delta$ 198.0, 159.5, 136.0, 132.9, 128.7 (2C), 128.3, 127.2, 126.4 (2C), 76.0, 73.7, 57.2, 51.6, 31.3, 29.7, 28.0, 21.1, 20.1.

IR (Diamond-ATR, neat) $\tilde{\mathrm{V}}_{\max }:$ 3260, 2926, 2855, 2153, 1761, 1715, 1444, 1169, 967, 745.

HRMS (ESI) m/z: [M+H] $]^{+}$Calcd for $\mathrm{C}_{19} \mathrm{H}_{20} \mathrm{NO}_{2}$ 294.1489; Found 294.1492. 
Feb12-2020-NM-20-053pure.15.fid
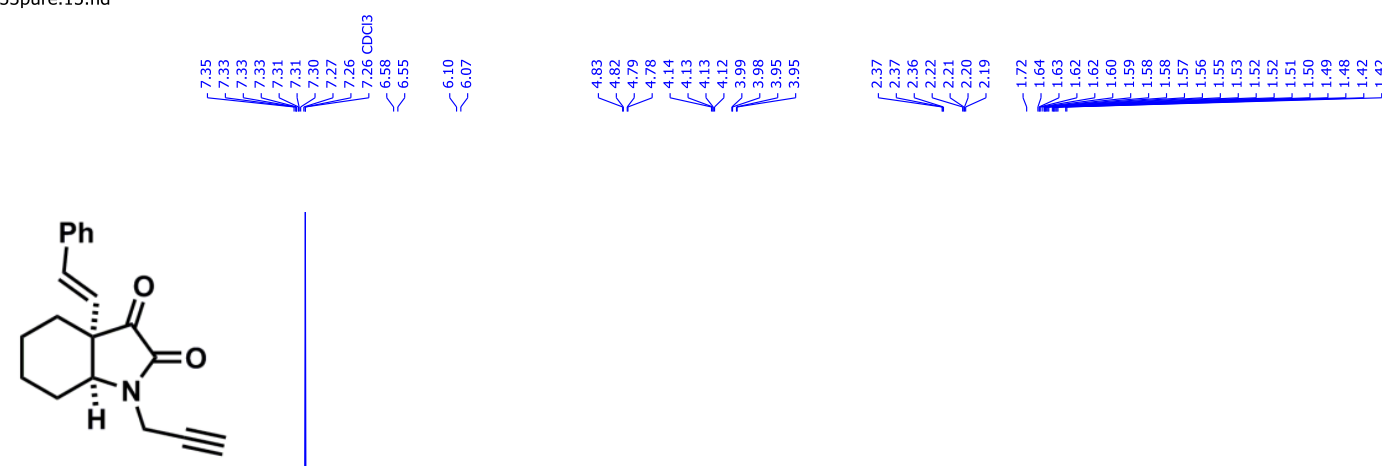

Solvent: $\mathrm{CDCl}_{3}$

Frequency: $500 \mathrm{MHz}$

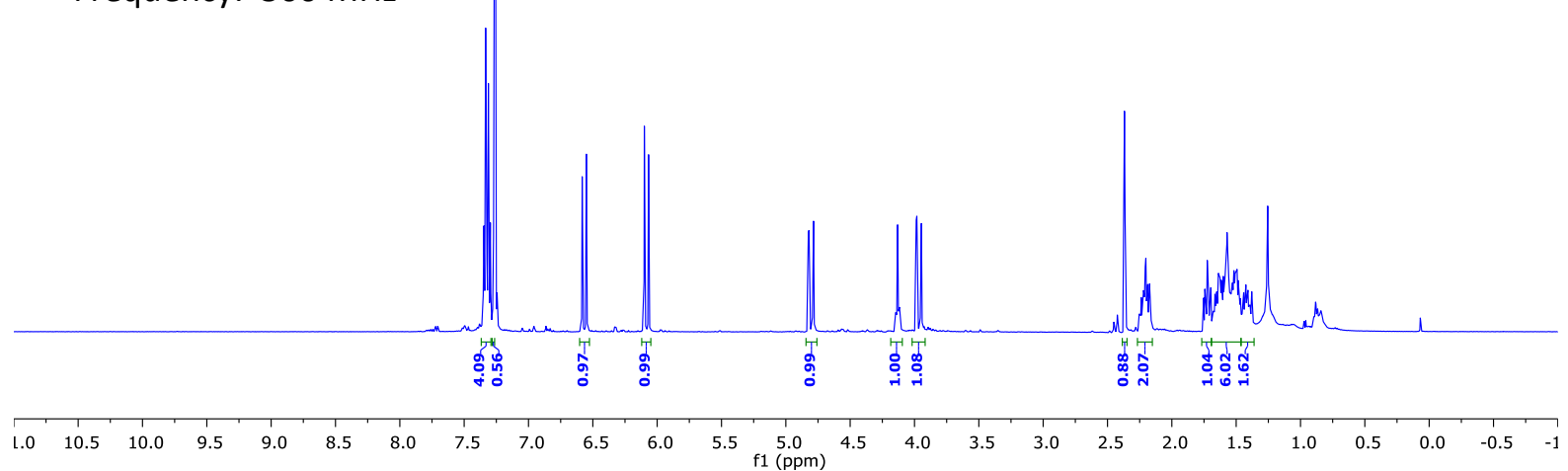

NM-20-053Rfo.6.14.fid

$\stackrel{8}{\circ}$
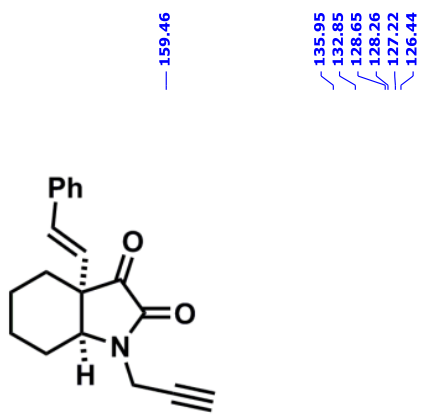

Solvent: $\mathrm{CDCl}_{3}$

Frequency: $176 \mathrm{MHz}$

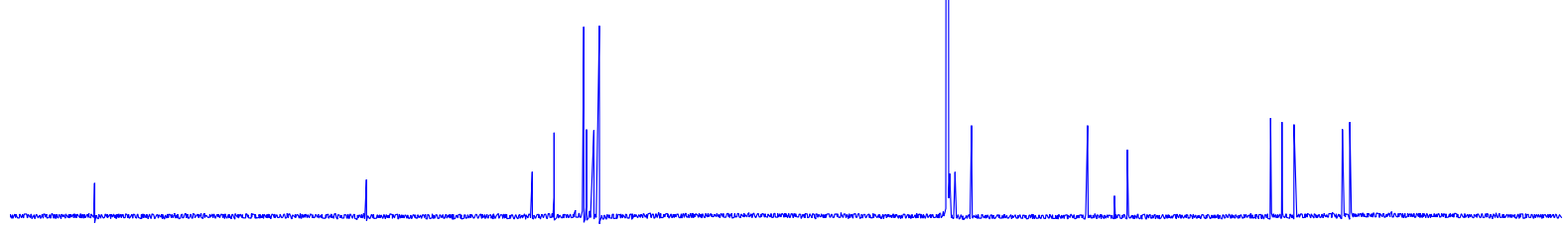

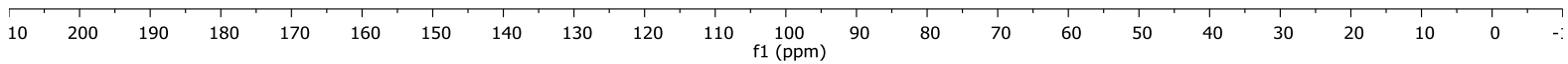


1-Methyl-3a-phenylhexahydro-1H-indole-2,3-dione (5i).

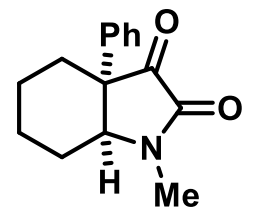

Yield: $6.3 \mathrm{mg}$, Quantitative.

Appearance: pale yellow oil.

$\boldsymbol{R}_{\mathrm{f}}=0.55$ (1:1 ethyl acetate:hexanes).

${ }^{1} \mathrm{H}$ NMR $\left(500 \mathrm{MHz}, \mathrm{CDCl}_{3}\right) \delta$ 7.38-7.32 (m, 2H), 7.31-7.27 (m, 3H), $4.19(\mathrm{t}, J=5.3 \mathrm{~Hz}, 1 \mathrm{H}), 3.11(\mathrm{~s}, 3 \mathrm{H}), 2.30$ (ddd, $J=14.4,6.2,4.5 \mathrm{~Hz}, 1 \mathrm{H}$ ), 2.18 (ddt, $J=13.7,9.1,4.8 \mathrm{~Hz}, 1 \mathrm{H}$ ), 1.90 (ddd, $J=14.2,9.3,4.9 \mathrm{~Hz}, 1 \mathrm{H}$ ), 1.67 (ddt, $J=18.2,12.6,5.5 \mathrm{~Hz}, 2 \mathrm{H}), 1.56-1.40(\mathrm{~m}, 3 \mathrm{H})$.

${ }^{13} \mathrm{C}$ NMR $\left(126 \mathrm{MHz}, \mathrm{CDCl}_{3}\right) \delta 200.1,139.1,129.1$ (2C), 127.3, 126.5 (2C), 60.4, 53.2, 30.9, 30.0, 29.7, 29.1, 20.8, 18.7 .

IR (Diamond-ATR, neat) $\tilde{v}_{\max }:$ 2922, 2853, 1759, 1713, 1664, 1612, 1506, 1452, 1208, 1034, 699.

HRMS (ESI) m/z: [M+H] ${ }^{+}$Calcd for $\mathrm{C}_{15} \mathrm{H}_{18} \mathrm{NO}_{2}$ 244.1332; Found 244.1335. 


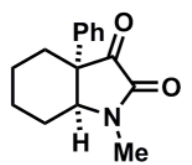

Solvent: $\mathrm{CDCl}_{3}$

Frequency: $500 \mathrm{MHz}$

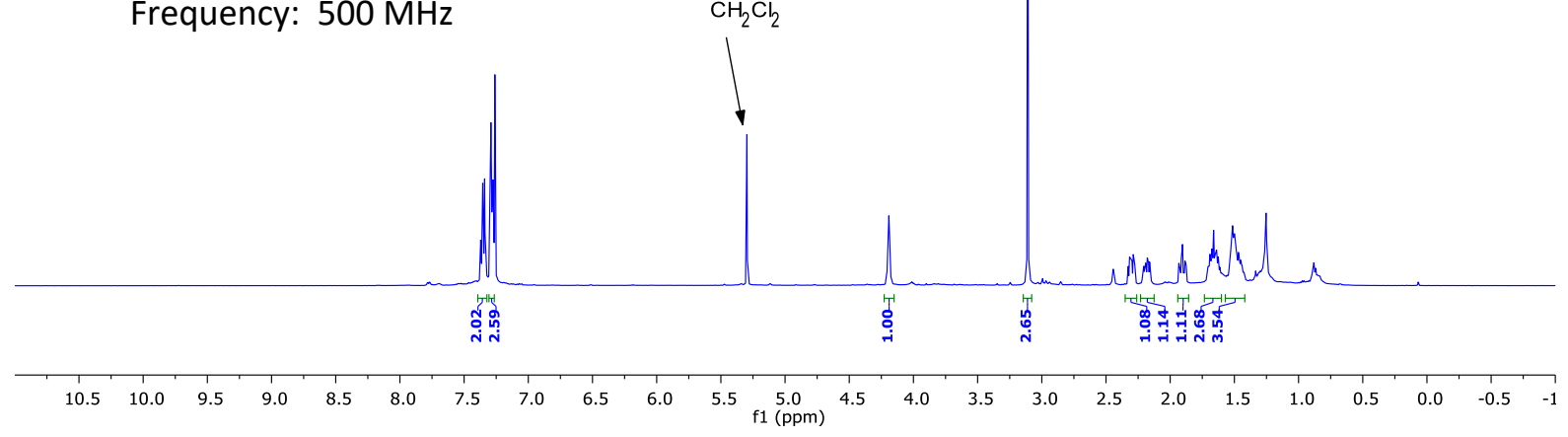

Mar19-2020-NM-20-078t10-12.13.fid

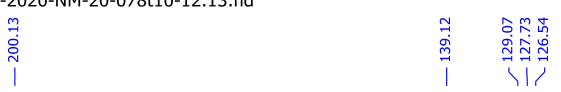

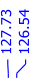

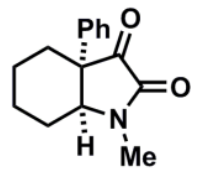

Solvent: $\mathrm{CDCl}_{3}$

Frequency: $126 \mathrm{MHz}$

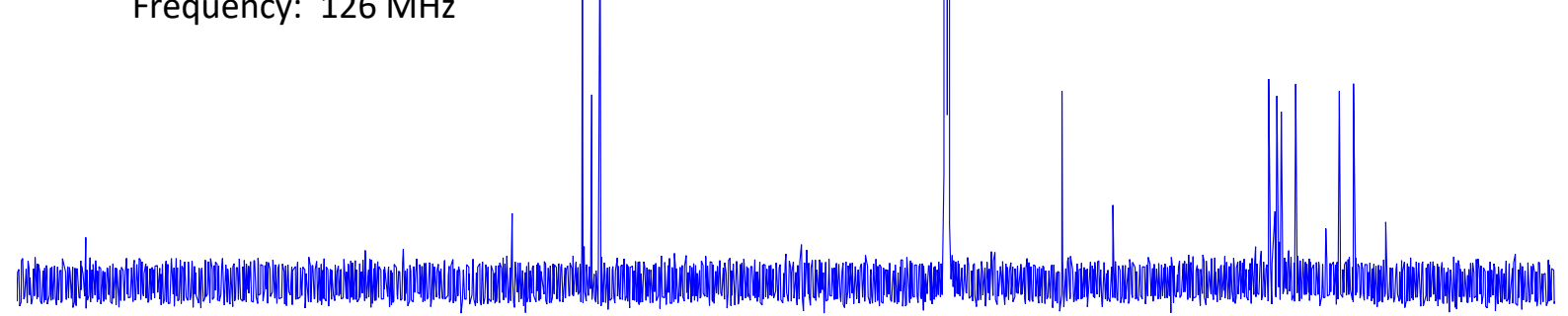

$10 \quad 200$

$150 \quad 140$

$\begin{array}{llll}130 & 120 & 110 & 100 \\ \mathrm{f} 1(\mathrm{ppm})\end{array}$

$80 \quad 70$

ำ

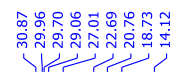




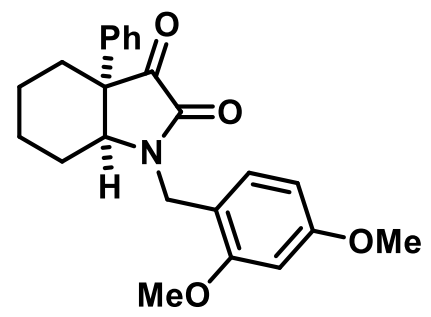

Yield: $6.0 \mathrm{mg}, 61 \% .273 .3 \mathrm{mg}, 57 \% .{ }^{17}$

Appearance: pale white grease.

$\boldsymbol{R}_{\mathrm{f}}=0.57$ (1:1 ethyl acetate:hexanes).

${ }^{1} \mathrm{H}$ NMR $\left(500 \mathrm{MHz}, \mathrm{CDCl}_{3}\right) \delta$ 7.24-7.14 (m, 3H), 7.06-6.96 (m, 3H), 6.48-6.32 (m, 2H), $5.10(\mathrm{~d}, J=14.4 \mathrm{~Hz}$, $1 \mathrm{H}), 4.22(\mathrm{~d}, J=14.5 \mathrm{~Hz}, 1 \mathrm{H}), 4.01(\mathrm{dd}, J=7.4,5.2 \mathrm{~Hz}, 1 \mathrm{H}), 3.81(\mathrm{~s}, 3 \mathrm{H}), 3.63(\mathrm{~s}, 3 \mathrm{H}), 2.43(\mathrm{dt}, J=14.1,4.3$ $\mathrm{Hz}, 1 \mathrm{H}), 2.28-2.18(\mathrm{~m}, 1 \mathrm{H}), 1.76-1.53(\mathrm{~m}, 3 \mathrm{H}), 1.45(\mathrm{ddt}, J=17.4,7.5,5.5 \mathrm{~Hz}, 1 \mathrm{H}), 1.41-1.30(\mathrm{~m}, 2 \mathrm{H})$.

${ }^{13} \mathrm{C}$ NMR $\left(126 \mathrm{MHz}, \mathrm{CDCl}_{3}\right) \delta 200.9,161.1,159.6,158.8,139.4,131.7,128.8$ (2C), 127.4, 126.3 (2C), 114.7, $104.3,98.4,57.0,55.4,55.2,54.0,40.1,31.5,29.1,21.4,19.8$.

IR (Diamond-ATR, neat) $\tilde{V}_{\max }: 2922,2850,1757,1705,1610,1586,1506,1456,1437,1418,1292,1265$, $1206,1155,1122,1031,835,732,697$.

HRMS (ESI) m/z: $[\mathrm{M}+\mathrm{H}]^{+}$Calcd for $\mathrm{C}_{23} \mathrm{H}_{26} \mathrm{NO}_{4}$ 380.1856; Found 380.1850.

[17] Synthesis of $\mathbf{5 j}$ on a $\mathbf{1 . 2 7} \mathbf{m m o l}$ scale. To a solution of $\mathbf{4 j} \mathbf{j}(700 \mathrm{mg}, 1.27 \mathrm{mmol}, 1.0$ equiv) in 32 $\mathrm{mL}$ dichloromethane $(0.04 \mathrm{M})$ was added anhydrous potassium carbonate $(1.05 \mathrm{~g}, 7.61 \mathrm{mmol}$, 6.0 equiv). Reaction was stirred at reflux for $24 \mathrm{~h}$ in an oil bath until full consumption of starting material was observed on TLC. The reaction was cooled to room temperature, diluted with 100 $\mathrm{mL}$ of dichloromethane and washed with $100 \mathrm{~mL}$ of saturated aqueous ammonium chloride solution and subsequently washed with $100 \mathrm{~mL}$ saturated aqueous sodium bicarbonate solution. Organic layer was filtered over sodium sulfate and concentrated to volume. Crude product was then purified by flash column chromatography eluting with a gradient of hexanes to $1: 1$ ethyl acetate:hexanes providing $5 \mathbf{j}$ ( $273.3 \mathrm{mg}, 57 \%)$. 


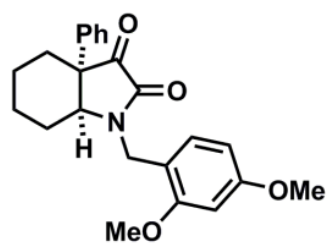

Solvent: $\mathrm{CDCl}_{3}$

Frequency: $500 \mathrm{MHz}$
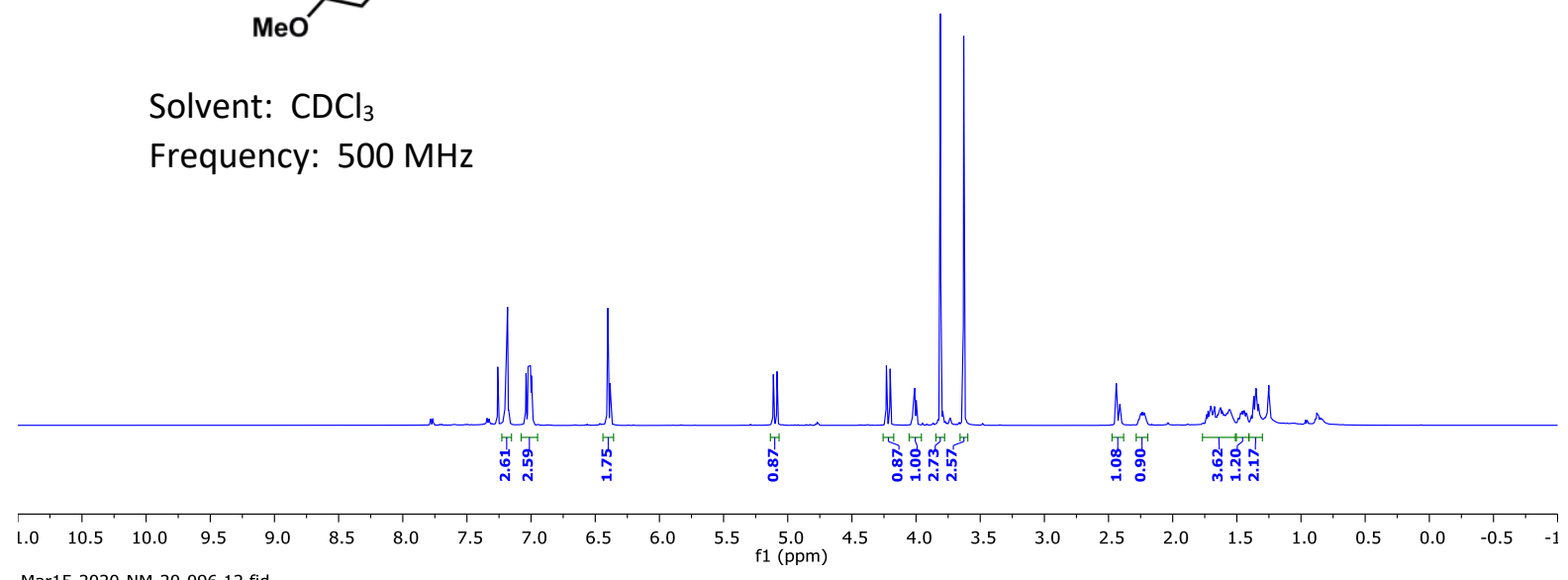
Mar15-2020-NM-20-096.12.fid
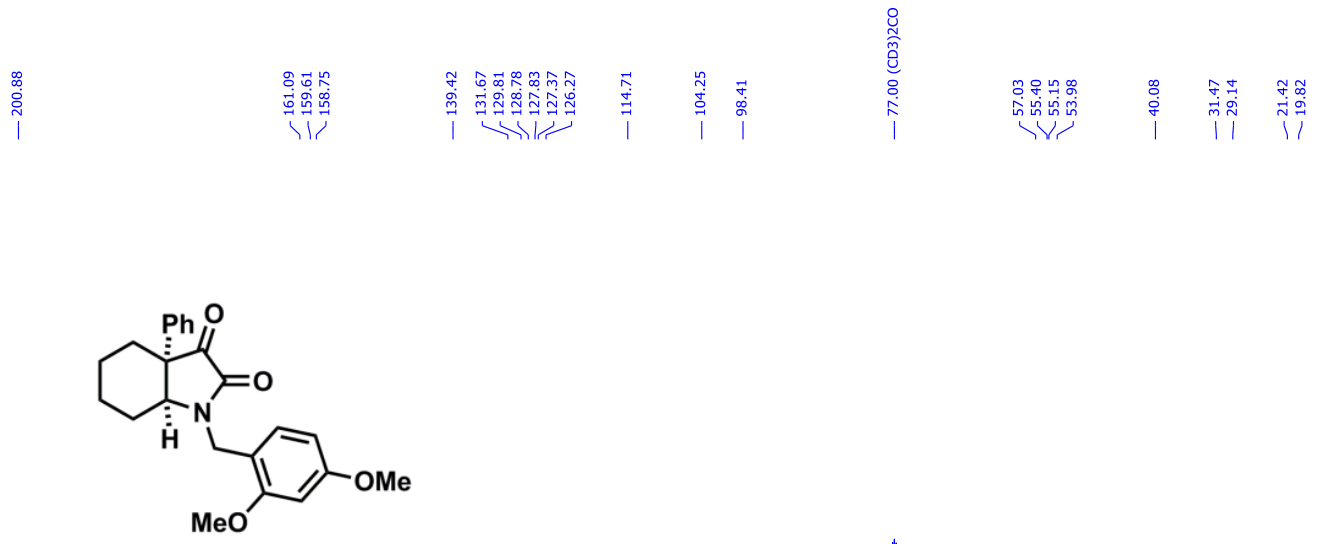

Solvent: $\mathrm{CDCl}_{3}$

Frequency: $126 \mathrm{MHz}$

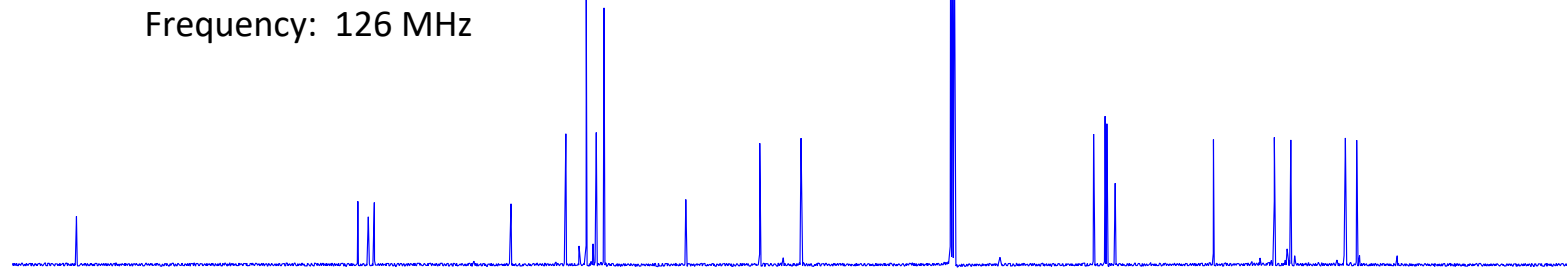

\begin{tabular}{lllllllllllllllllllllll}
\hline 10 & 200 & 190 & 180 & 170 & 160 & 150 & 140 & 130 & 120 & 110 & $\begin{array}{c}100 \\
\mathrm{f} 1(\mathrm{ppm})\end{array}$ & 90 & 80 & 70 & 60 & 50 & 40 & 30 & 20 & 10 & 0 & $-:$
\end{tabular} 


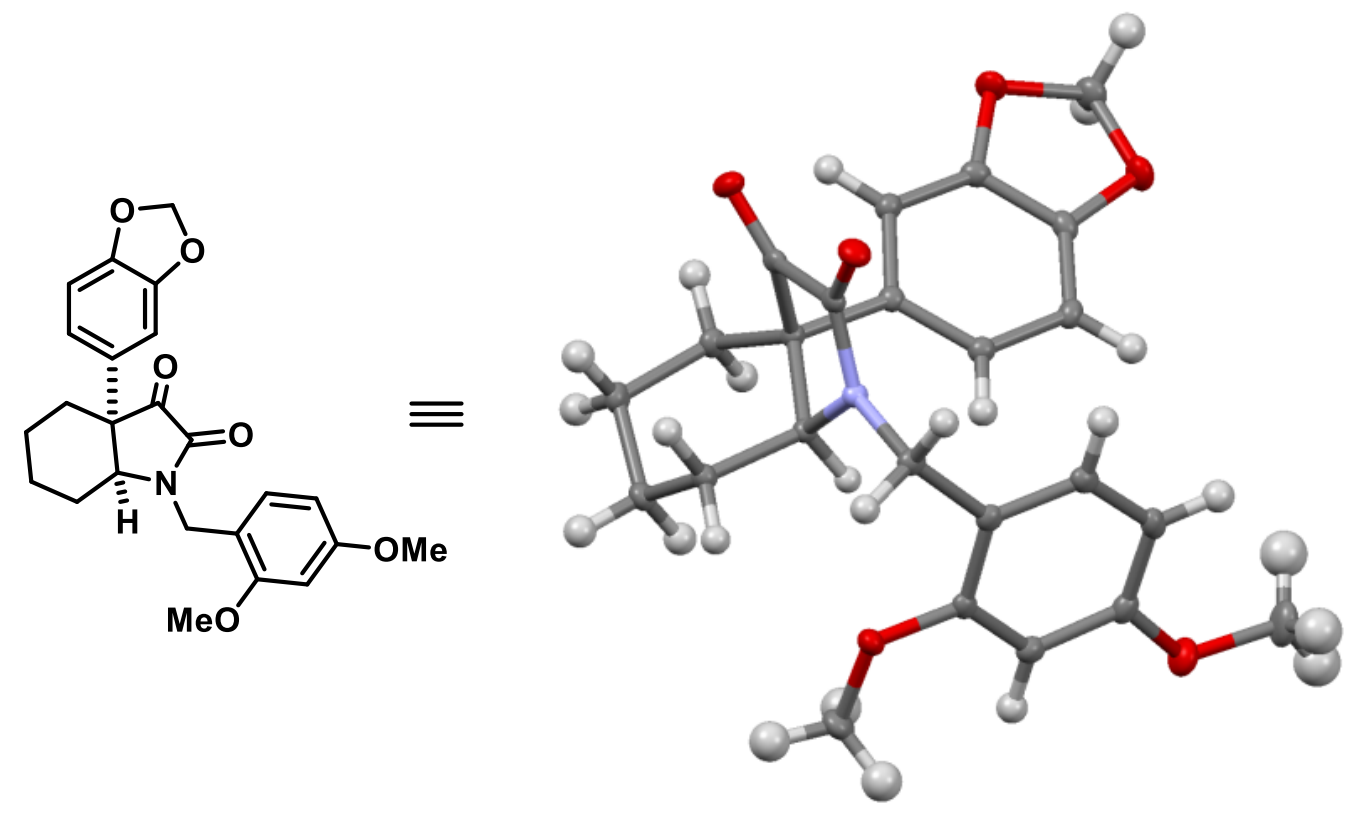

Yield: $8.6 \mathrm{mg}, 78 \% .(87 \%)^{18}$

Appearance: pale yellow viscous oil.

$\boldsymbol{R}_{\mathbf{f}}=0.45$ (1:1 ethyl acetate:hexanes).

${ }^{1} \mathrm{H}$ NMR $\left(700 \mathrm{MHz}, \mathrm{CDCl}_{3}\right) \delta 7.08(\mathrm{~d}, \mathrm{~J}=8.2 \mathrm{~Hz}, 1 \mathrm{H}), 6.60(\mathrm{~d}, \mathrm{~J}=8.2 \mathrm{~Hz}, 1 \mathrm{H}), 6.50(\mathrm{~d}, \mathrm{~J}=2.0 \mathrm{~Hz}, 1 \mathrm{H}), 6.46-$ $6.43(\mathrm{~m}, 2 \mathrm{H}), 6.42(\mathrm{dd}, \mathrm{J}=8.3,2.4 \mathrm{~Hz}, 1 \mathrm{H}), 5.90(\mathrm{dd}, \mathrm{J}=5.8,1.4 \mathrm{~Hz}, 2 \mathrm{H}), 5.10(\mathrm{~d}, \mathrm{~J}=14.4 \mathrm{~Hz}, 1 \mathrm{H}), 4.21(\mathrm{~d}, \mathrm{~J}$ $=14.4 \mathrm{~Hz}, 1 \mathrm{H}), 3.92(\mathrm{dd}, \mathrm{J}=7.5,5.2 \mathrm{~Hz}, 1 \mathrm{H}), 3.82(\mathrm{~s}, 3 \mathrm{H}), 3.72(\mathrm{~s}, 3 \mathrm{H}), 2.38(\mathrm{dt}, \mathrm{J}=13.6,4.1 \mathrm{~Hz}, 1 \mathrm{H}), 2.24-$ $2.19(\mathrm{~m}, 1 \mathrm{H}), 1.70-1.58(\mathrm{~m}, 2 \mathrm{H}), 1.58-1.52(\mathrm{~m}, 1 \mathrm{H}), 1.47-1.41(\mathrm{~m}, 1 \mathrm{H}), 1.36-1.31(\mathrm{~m}, 2 \mathrm{H})$.

${ }^{13} \mathrm{C}$ NMR $\left(176 \mathrm{MHz}, \mathrm{CDCl}_{3}\right) \delta 200.6,161.2,159.6,158.8,148.1,146.8,133.0,131.9,119.8,114.7,108.2$, $106.9,104.3,101.2,98.5,57.3,55.4,55.3,53.6,40.1,31.4,29.1,21.4,19.8$.

IR (Diamond-ATR, neat) $\tilde{V}_{\text {max }}: 2935,2859,1756,1703,1610,1586,1504,1435,1237,1206,1155,1120$, 1031, 928, 811, 731.

HRMS (ESI) m/z: [M+H] $]^{+}$Calcd for $\mathrm{C}_{24} \mathrm{H}_{26} \mathrm{NO}_{6}$ 424.1755; Found 424.1745.

X-ray sample preparation: Compound $\mathbf{5 I}$ contained in a $20 \mathrm{~mL}$ scintillation vial was dissolved in $15 \mathrm{~mL}$ of chloroform. This vial was attached to a rotoevaporator with applied vacuum, slow rotation and no heating bath. After partial evaporation of approximately $5 \mathrm{~mL}$ of chloroform, the cold solution of $\mathbf{5 I}$ precipitated crystals. These crystals were a A colorless plate-like specimen of $\mathrm{C}_{24} \mathrm{H}_{25} \mathrm{NO}_{6}$, approximate dimensions $0.040 \mathrm{~mm} \times 0.111 \mathrm{~mm} \times 0.206 \mathrm{~mm}$, was used for the X-ray crystallographic analysis. The Xray intensity data were measured on a Bruker D8 VENTURE system equipped with an Incoatec I $\mu \mathrm{S} 3.0$ microfocus sealed tube ( $\mathrm{Cu} \mathrm{K \alpha}, \lambda=1.54178 \AA$ ) and a multilayer mirror monochromator.

The total exposure time was 6.63 hours. The frames were integrated with the Bruker SAINT software package using a narrow-frame algorithm. The integration of the data using a triclinic unit cell yielded a 
total of 33549 reflections to a maximum $\theta$ angle of $79.92^{\circ}$ ( $0.78 \AA$ resolution), of which 4263 were independent (average redundancy 7.870, completeness $=98.2 \%, R_{\text {int }}=2.53 \%, R_{\text {sig }}=1.49 \%$ ) and $4047(94.93 \%)$ were greater than $2 \sigma\left(F^{2}\right)$. The final cell constants of $\underline{a}=10.1737(7) \AA, \underline{b}=10.6211(8) \AA, \underline{c}=11.2151(9) \AA, \alpha=94.945(4)^{\circ}, \beta=109.447(4)^{\circ}, \gamma=115.494(4)^{\circ}$, volume $=993.25(13) \AA^{3}$, are based upon the refinement of the XYZ-centroids of 582 reflections above 20 $\sigma(I)$ with $8.864^{\circ}<2 \theta<101.6^{\circ}$. Data were corrected for absorption effects using the Multi-Scan method (SADABS). The ratio of minimum to maximum apparent transmission was 0.933 . The calculated minimum and maximum transmission coefficients (based on crystal size) are 0.7036 and 0.7543 .

The structure was solved and refined using the Bruker SHELXTL Software Package, using the space group P -1 , with $\mathrm{Z}=2$ for the formula unit, $\mathrm{C}_{24} \mathrm{H}_{25} \mathrm{NO}_{6}$. The final anisotropic full-matrix least-squares refinement on $F^{2}$ with 282 variables converged at $R 1=3.19 \%$, for the observed data and $\mathrm{WR} 2=8.24 \%$ for all data. The goodness-of-fit was 1.021. The largest peak in the final difference electron density synthesis was $0.309 \mathrm{e}^{-}$ $/ \AA^{3}$ and the largest hole was $-0.224 \mathrm{e}^{-} / \AA^{3}$ with an RMS deviation of $0.041 \mathrm{e}^{-} / \AA^{3}$. On the basis of the final model, the calculated density was $1.416 \mathrm{~g} / \mathrm{cm}^{3}$ and $F(000), 448 \mathrm{e}^{-}$.

CCDC 1991570

[18] Reaction was performed at a $0.32 \mathrm{mmol}$ scale. 


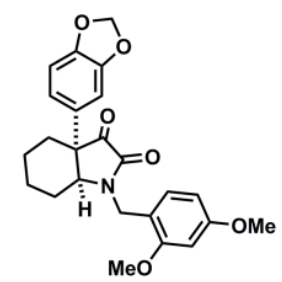

Solvent: $\mathrm{CDCl}_{3}$

Frequency: $700 \mathrm{MHz}$

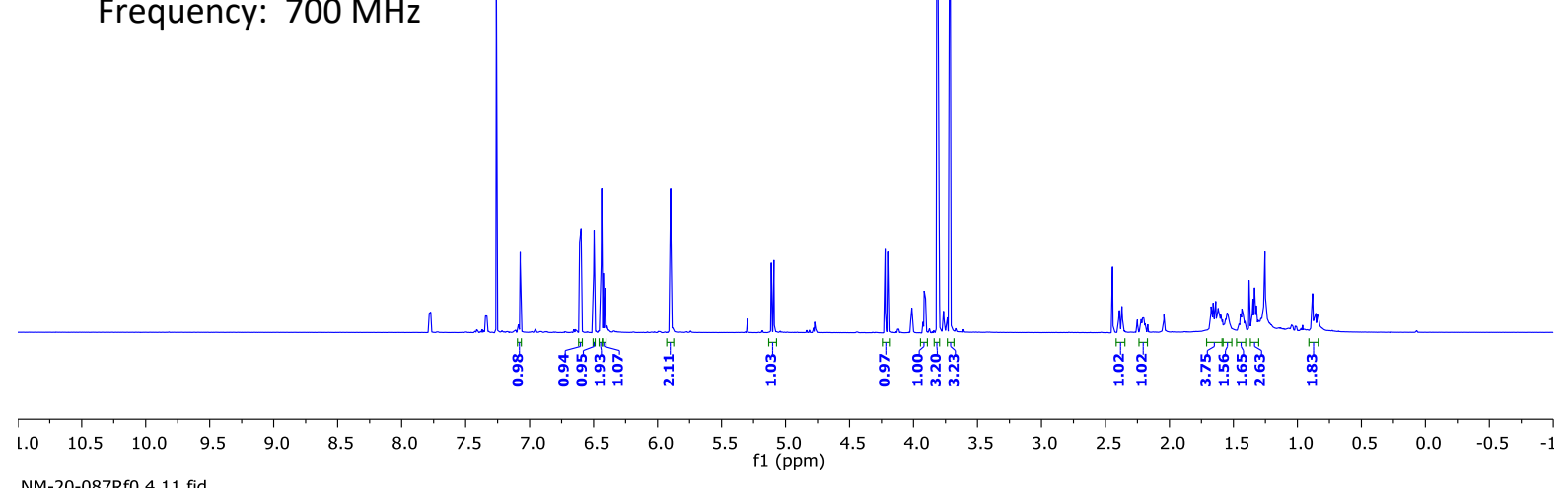
NM-20-087Rf0.4.11.fid

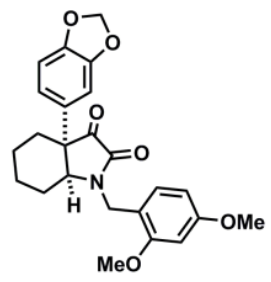

Solvent: $\mathrm{CDCl}_{3}$

Frequency: $176 \mathrm{MHz}$
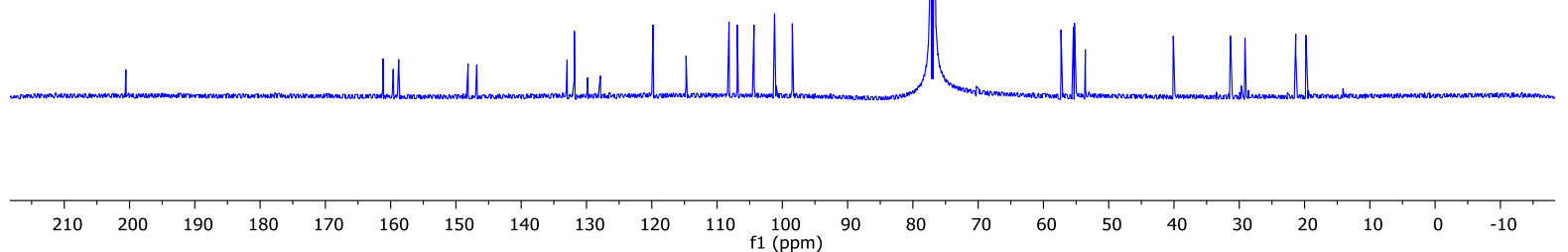


\begin{tabular}{|c|c|c|}
\hline Identification code & \multicolumn{2}{|l|}{ rds998 } \\
\hline Chemical formula & \multicolumn{2}{|l|}{$\mathrm{C}_{24} \mathrm{H}_{25} \mathrm{NO}_{6}$} \\
\hline Formula weight & \multicolumn{2}{|l|}{$423.45 \mathrm{~g} / \mathrm{mol}$} \\
\hline Temperature & \multicolumn{2}{|l|}{$101(2) \mathrm{K}$} \\
\hline Wavelength & \multicolumn{2}{|l|}{$1.54178 \AA$} \\
\hline Crystal size & \multicolumn{2}{|c|}{$0.040 \times 0.111 \times 0.206 \mathrm{~mm}$} \\
\hline Crystal habit & \multicolumn{2}{|l|}{ colorless plate } \\
\hline Crystal system & \multicolumn{2}{|l|}{ triclinic } \\
\hline Space group & \multicolumn{2}{|l|}{$P-1$} \\
\hline \multirow[t]{3}{*}{ Unit cell dimensions } & $a=10.1737(7) \AA$ & $\alpha=94.945(4)^{\circ}$ \\
\hline & $b=10.6211(8) \AA$ & $\beta=109.447(4)^{\circ}$ \\
\hline & $c=11.2151(9) \AA$ & $\gamma=115.494(4)^{\circ}$ \\
\hline Volume & \multicolumn{2}{|l|}{$993.25(13) \AA^{3}$} \\
\hline $\mathbf{Z}$ & \multicolumn{2}{|l|}{2} \\
\hline Density (calculated) & \multicolumn{2}{|l|}{$1.416 \mathrm{~g} / \mathrm{cm}^{3}$} \\
\hline Absorption coefficient & \multicolumn{2}{|l|}{$0.840 \mathrm{~mm}^{-1}$} \\
\hline$F(000)$ & \multicolumn{2}{|l|}{448} \\
\hline
\end{tabular}


Table 2. Data collection and structure refinement for 51.

\begin{tabular}{|c|c|c|}
\hline Diffractometer & \multicolumn{2}{|l|}{ Bruker D8 VENTURE } \\
\hline Radiation source & \multicolumn{2}{|c|}{$\begin{array}{l}\text { Incoatec I } \mu \text { S } 3.0 \text { microfocus sealed tube (Cu K } \alpha, \lambda \\
=1.54178 \AA \AA)\end{array}$} \\
\hline $\begin{array}{l}\text { Theta range for data } \\
\text { collection }\end{array}$ & \multicolumn{2}{|l|}{4.34 to $79.92^{\circ}$} \\
\hline Index ranges & \multicolumn{2}{|c|}{$-12<=\mathrm{h}<=12,-13<=\mathrm{k}<=13,-14<=\mathrm{|}<=14$} \\
\hline Reflections collected & \multicolumn{2}{|c|}{33549} \\
\hline Independent reflections & \multicolumn{2}{|c|}{$4263[\mathrm{R}$ (int) $=0.0253]$} \\
\hline $\begin{array}{l}\text { Coverage of independent } \\
\text { reflections }\end{array}$ & \multicolumn{2}{|l|}{$98.2 \%$} \\
\hline Absorption correction & \multicolumn{2}{|l|}{ Multi-Scan } \\
\hline $\begin{array}{l}\text { Max. and min. } \\
\text { transmission }\end{array}$ & \multicolumn{2}{|l|}{0.7543 and 0.7036} \\
\hline $\begin{array}{l}\text { Structure solution } \\
\text { technique }\end{array}$ & \multicolumn{2}{|l|}{ direct methods } \\
\hline $\begin{array}{l}\text { Structure solution } \\
\text { program }\end{array}$ & \multicolumn{2}{|c|}{ XT, VERSION 2014/5 (Sheldrick, 2015) } \\
\hline Refinement method & \multicolumn{2}{|c|}{ Full-matrix least-squares on $\mathrm{F}^{2}$} \\
\hline Refinement program & \multicolumn{2}{|c|}{ SHELXL-2018/3 (Sheldrick, 2015) } \\
\hline Function minimized & \multicolumn{2}{|c|}{$\Sigma w\left(F_{o}^{2}-F_{c}^{2}\right)^{2}$} \\
\hline $\begin{array}{l}\text { Data / restraints / } \\
\text { parameters }\end{array}$ & \multicolumn{2}{|l|}{4263 / 0 / 282} \\
\hline Goodness-of-fit on $\mathrm{F}^{2}$ & \multicolumn{2}{|l|}{1.021} \\
\hline \multirow[t]{2}{*}{ Final $\mathbf{R}$ indices } & 4047 data; $1>2 \sigma(I)$ & $\mathrm{R} 1=0.0319, \mathrm{wR} 2=0.0810$ \\
\hline & all data & $\mathrm{R} 1=0.0333, \mathrm{wR} 2=0.0824$ \\
\hline Weighting scheme & \multicolumn{2}{|c|}{$\begin{array}{l}w=1 /\left[\sigma^{2}\left(F_{o}^{2}\right)+(0.0388 P)^{2}+0.4372 P\right] \\
\text { where } P=\left(F_{o}^{2}+2 F_{c}^{2}\right) / 3\end{array}$} \\
\hline
\end{tabular}

Largest diff. peak and hole 0.309 and $-0.224 \mathrm{e}^{-3}$

R.M.S. deviation from mean

$0.041 \mathrm{e}^{-3}$ 


\section{(E)-1-(2,4-diMethoxybenzyl)-3a-styrylhexahydrocyclopenta[b]pyrrole-2,3-dione (5n).}

A solution of the corresponding pyruvic methyl ester $\mathbf{1 a}$ (1.0 equiv) and aldehyde $\mathbf{2 b}$ (1.1 equiv) in anhydrous dichloromethane $(0.5 \mathrm{M})$ was added primary amine $3 a$ (1.1 equiv) at $0{ }^{\circ} \mathrm{C}$. Reaction was allowed to stir to room temperature. Reaction was monitored by TLC for full consumption of pyruvic methyl ester 1a. Upon completion, reaction was concentrated to volume and immediately purified by flash column chromatography using a gradient from hexanes to 1:1 ethyl acetate:hexanes mixture yielding pyrrole intermediate that was directly utilized in general procedure 3 for the synthesis of $\mathbf{5 n}$.

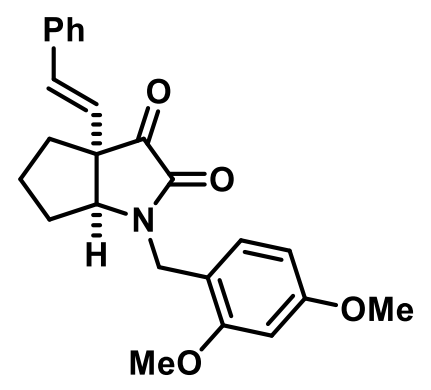

Yield: $2.0 \mathrm{mg}, 23 \%$.

Appearance: orange grease.

$\boldsymbol{R}_{\mathbf{f}}=0.45$ (1:1 ethyl acetate:hexanes).

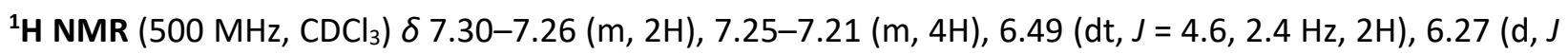
$=16.2 \mathrm{~Hz}, 1 \mathrm{H}), 6.09(\mathrm{~d}, J=16.2 \mathrm{~Hz}, 1 \mathrm{H}), 5.15(\mathrm{~d}, J=14.3 \mathrm{~Hz}, 1 \mathrm{H}), 4.33(\mathrm{~d}, J=14.4 \mathrm{~Hz}, 1 \mathrm{H}), 3.99(\mathrm{~d}, J=6.5$ $\mathrm{Hz}, 1 \mathrm{H}), 3.82(\mathrm{~s}, 3 \mathrm{H}), 3.80(\mathrm{~s}, 3 \mathrm{H}), 2.34(\mathrm{dd}, J=11.4,6.0 \mathrm{~Hz}, 1 \mathrm{H}), 2.00(\mathrm{dd}, J=12.5,6.1 \mathrm{~Hz}, 1 \mathrm{H}), 1.83-1.73$ (m, 3H), 1.48-1.41 (m, 1H).

${ }^{13} \mathrm{C} \mathrm{NMR}\left(176 \mathrm{MHz}, \mathrm{CDCl}_{3}\right) \delta 202.1,161.2,159.2,158.8,136.1,131.7,131.1,128.6(2 \mathrm{C}), 128.0,126.9,126.3$ (2C), 115.2, 104.6, 98.6, 63.4, 57.5, 55.5, 55.4, 41.0, 37.3, 30.3, 24.0.

IR (Diamond-ATR, neat) $\tilde{V}_{\text {max: }}$ 2993, 2967, 2881, 1838, 1741, 1640, 1536, 1226, 950, 723.

HRMS (ESI) m/z: [M+H] $]^{+}$Calcd for $\mathrm{C}_{24} \mathrm{H}_{26} \mathrm{NO}_{4}$ 392.1856; Found 392.1860. 


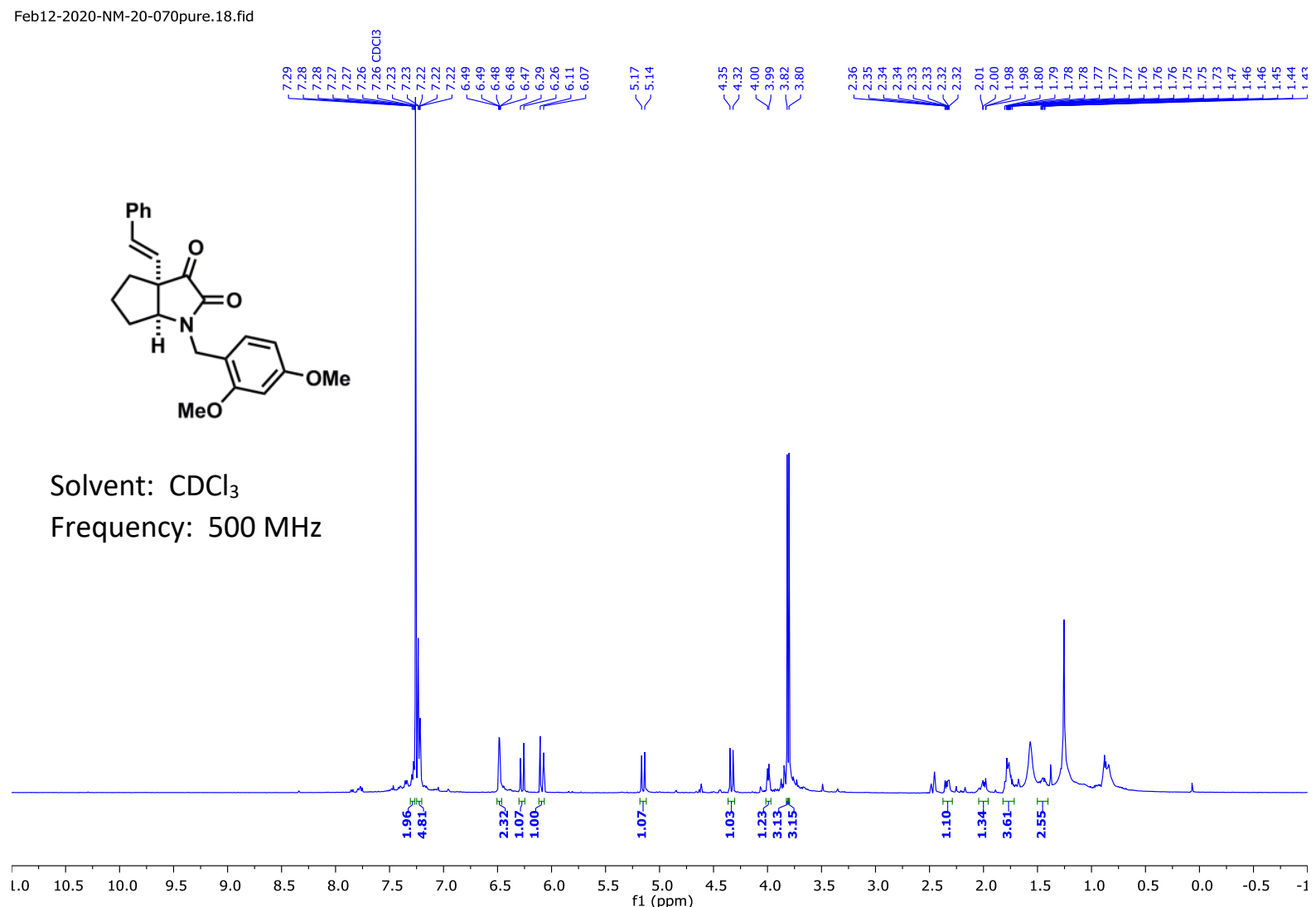

NM-20-070.13.fid

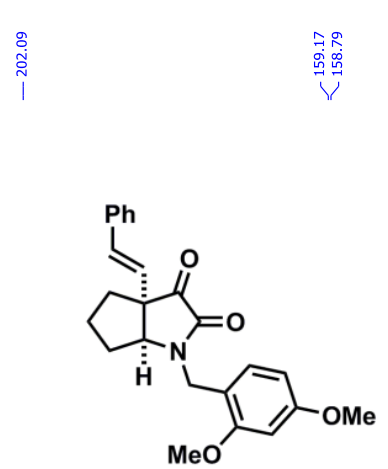

Solvent: $\mathrm{CDCl}_{3}$

Frequency: $176 \mathrm{MHz}$
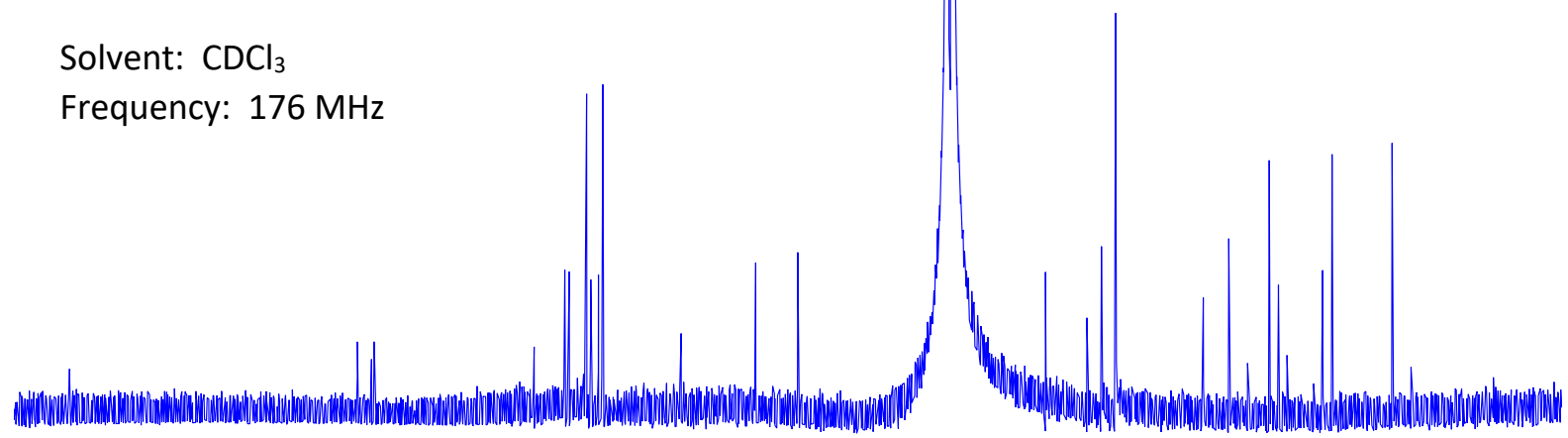

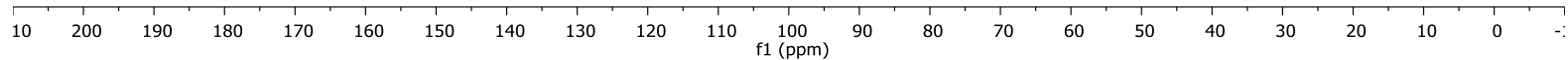

